\title{
Complete Boolean algebras of type I factors
}

\author{
By
}

\author{
Huzihiro Araki and E. J. Woods*†
}

\begin{abstract}
A partial classification into unitary equivalence classes of complete Boolean algebras of type I factors is given. Any complete atomic Boolean algebra of type I factors is unitarily equivalent to a discrete tensor product of type I factors. We establish a one-to-one correspondence between the unitary equivalence classes of complete nonatomic Boolean algebras of type I factors satisfying a certain condition, and the unitary equivalence classes of complete nonatomic Boolean algebras of projections. A continuous tensor product of Hilbert spaces is defined which is a generalization of the discrete infinite incomplete tensor product space defined by von Neumann. On a separable Hilbert space, any complete nonatomic Boolean algebra of type I factors satisfying the previously mentioned condition is unitarily equivalent to a continuous tensor product of type I factors. An application to the representations of the canonical commutation relations of quantum field theory is made.
\end{abstract}

\section{Introduction}

In this paper we give a partial classification (up to unitary equivalence) of complete Boolean algebras of type I factors. We also give an application of these results to the group integral for irreducible representations of the canonical commutation relations (hereafter referred to as CCRs). The mathematical motivation for this problem is provided by the work of

Received April 30, 1966.

* Supported in part by National Science Foundation Grant GP 3221.

$\dagger$ Department of Physics and Astronomy, University of Maryland, College Park, Maryland U.S.A. 
Murray and von Neumann ${ }^{1)}$ on direct factorizations (finite type I factorizations in our terminology), and by von Neumann's construction of an incomplete tensor product of Hilbert spaces (hereafter referred to as an ITPS). ${ }^{2} \quad$ Further motivation is provided by recent work in mathematical physics where lattices of von Neumann algebras have been studied. ${ }^{\left.3{ }^{3} 4\right)}$

We begin with an outline of the general mathematical problem (even though our results are only a first step towards the understanding of such structures). We give a few definitions from lattice theory. A lattice $E$ is a partially ordered set any two of whose elements have a greatest lower bound $x \wedge y$ and least upper bound $x \vee y$. A lattice is said to be complete if every subset has a greatest lower bound and least upper bound. We consider lattices $E$ which contain (necessarily unique) elements 0 and 1 satisfying $0 \leqslant x \leqslant 1$ for all $x \in E$. Any $x \neq 0$ such that $x>y$ implies $y=0$ is called an atom. An atomic lattice is one in which every $x \neq 0$ includes at least one atom. An involution is a map $x \rightarrow x^{\prime}$ satisfying

(i) $x=x^{\prime \prime}=\left(x^{\prime}\right)^{\prime}$

(ii) $(x \wedge y)^{\prime}=x^{\prime} \vee y^{\prime}$ and $(x \vee y)^{\prime}=x^{\prime} \wedge y^{\prime}$

A complemented lattice is a lattice in which every element $x$ has a complement $y$ such that $x \wedge y=0, x \vee y=1$. An orthocomplementation is an involution $x \rightarrow x^{\prime}$ where $x^{\prime}$ is a complement of $x$.

We now give some examples. Let $H$ be a Hilbert space, $\mathrm{B}(H)$ the set of all bounded linear operators on $H$. If $A \subset \mathrm{B}(H)$ we define its commutor

$$
A^{\prime}=\left\{Q^{\prime} \in \mathrm{B}(H): \quad Q Q^{\prime}=Q^{\prime} Q \text { for all } Q \in A\right\}
$$

1) F.J. Murray and J. von Neumann, Ann. Math. 37, 116 (1936).

2) J. von Neumann, Compositio Math. 6, 1 (1938).

3) For a detailed account of the role of von Neumann algebras of local observables in relativistic quantum field theory see for example R. Haag, Proceedings of the Midwest Conference on Theoretical Physics, Minneapolis, 1961; R. Haag and B. Schroer, J. Math. Phys. 3, 248 (1962); H. Araki, ETH lecture notes (Zurich, 1961/62), and to be published by W. Benjamin, New York; R. Haag and D. Kastler, J. Math. Phys. 5, 848 (1964).

4) For a detailed account of the lattice of von Neumann algebras associated with the quantum theory of a free Bose field see H. Araki, J. Math. Phys. 4, 1343 (1963). 
Clearly $A \subset A^{\prime \prime}=\left(A^{\prime}\right)^{\prime}$. The set $A$ is called self-adjoint if $T \in A$ implies $T^{*} \in A$. The set $A$ is called a von Neumann algebra if it is self-adjoint and $A=A^{\prime \prime}$. Given an arbitrary self-adjoint set $S \subset \mathrm{B}(H), S^{\prime \prime}$ is the von Neumann algebra generated by the set $S$. The set of all von Neumann algebras $R \subset \mathrm{B}(H)$ forms an involutive lattice where the lattice operations are defined by

$$
\begin{aligned}
& R_{1} \wedge R_{2}=R_{1} \cap R_{2} \\
& R_{1} \vee R_{2}=\left\{R_{1} \cup R_{2}\right\}^{\prime \prime}
\end{aligned}
$$

where $\cap, U$ denotes the set theoretical intersection and union respectively, and the involution is given by $R \rightarrow R^{\prime}$ This lattice has a maximal element $\mathrm{B}(H)$ and a minimal element $\mathrm{B}(H)^{\prime}=\{\alpha \mathbb{1}\}$ (the set of all multiples of the identity operator). The usual notation for lattices would suggest that we denote these von Neumann algebras by 1 and 0 respectively. However, the standard notation is to write $\mathbb{1}$ for $\{\alpha \mathbb{1}\}$. Hence we shall use $\mathbb{1}$ and $\mathrm{B}(H)$ for the minimal and maximal elements of this lattice. $\mathrm{A}$ von Neumann algebra satisfying

$$
R \wedge R^{\prime}=\mathbb{1}
$$

or equivalently

$$
R \vee R^{\prime}=\mathrm{B}(H)
$$

is called a factor. The set of all factors is not a lattice since in general if $R_{1}, R_{2}$ are factors then $R_{1} \vee R_{2}$ and $R_{1} \wedge R_{2}$ are not factors. However, if $E$ is any lattice of factors then the map $R \rightarrow R^{\prime}$ is an orthocomplementation in $E$.

A representation of an orthocomplemented lattice $E$ by von Neumann algebras is the structure consisting of a Hilbert space $H$ and a map $x \rightarrow \mathrm{R}(x)$ from $E$ into the lattice of von Neumann algebras on $H$ such that

$$
\begin{aligned}
& \mathrm{R}(x \vee y)=\mathrm{R}(x) \vee \mathrm{R}(y) \\
& \mathrm{R}(x \wedge y)=\mathrm{R}(x) \wedge \mathrm{R}(y) \\
& \mathrm{R}\left(x^{\prime}\right)=\mathrm{R}(x)^{\prime}
\end{aligned}
$$

The Fock representation of the CCRs over a real Hilbert space $K$ provides an example of such a representation. To every subspace (closed linear 
subset) $W$ of the real Hilbert space $K$ there corresponds a von Neumann algebra $\mathrm{R}_{\mathrm{F}}(W)$ on a complex Hilbert space (the Fock space $\mathrm{H}_{\mathrm{F}}(K)$ ). A detailed investigation of this example has been given by Araki, ${ }^{4)}$ who proved that the map $W \rightarrow \mathrm{R}_{\mathrm{F}}(W)$ gives an isomorphism of the involutive lattice of subspaces of $K$ with the involutive lattice of von Neumann algebras $\mathrm{R}_{\mathrm{F}}(W)$. It should be noted that the duality theorem, Eq. (1. 7), is the most difficult property to prove in this isomorphism.

A distributive lattice $E$ is one that satisfies

$$
\begin{aligned}
& x \vee(y \wedge z)=(x \vee y) \wedge(x \vee z) \\
& x \wedge(y \vee z)=(x \wedge y) \vee(x \wedge z)
\end{aligned}
$$

for all $x, y, z \in E$. A Boolean algebra is a complemented distributive lattice. The distributive property implies that any $x$ has a unique complement $x^{\prime}$. Thus a Boolean algebra is a special case of an orthocomplemented lattice. We now give an example of an atomic Boolean algebra of factors.

Definition 1. 1: Let $H$ be a Hilbert space and $R$ a von Neumann algebra in $\mathrm{B}(H)$. We say that $\left\{R_{a b}\right\}_{a \in A}$ is a factorization of $R$ if each $R_{a}$ is a factor and

$$
\begin{aligned}
& R_{\alpha} \subset R_{\beta}{ }^{\prime}, \quad \alpha \neq \beta \\
& \bigvee_{\alpha \in A} R_{\alpha}=R
\end{aligned}
$$

If $R=\mathrm{B}(H)$ we just say that $\left\{R_{\alpha}\right\}_{a \in A}$ is a factorization. If $A$ is a finite (countable) set, we say that $\left\{R_{\alpha}\right\}_{\omega \in A}$ is a finite (countable) factorization. If each $R_{\alpha}$ is a type I factor, we say that $\left\{R_{\alpha}\right\}_{\alpha \in A}$ is a type I factorization.

Given a factorization $\left\{R_{a i}\right\}_{a \in A}$, and a subset $J \subset A$, let $J^{\mathrm{c}}=A-J$ and define

$$
\mathrm{R}(J)=\bigvee_{\alpha \in I} R_{a i}, \quad \mathrm{R}(\phi)=\mathbb{1}
$$

Since $\mathrm{R}(A)=\mathrm{B}(H)$ and $\mathrm{R}(J)^{\prime} \supset \mathrm{R}\left(J^{\mathrm{c}}\right)$ we have

$$
\mathrm{R}(J) \vee \mathrm{R}(J)^{\prime}=\mathrm{B}(H)
$$

5) This follows from lemma 5.4.1 of F.J. Murray and J. von Neumann (reference 1). This lemma is stated only for separable $\mathrm{H}$, but can easily be generalized to the nonseparable case. 
and $\mathrm{R}(J)$ is a factor. We say the factorization is coupled if $\mathrm{R}(J)^{\prime}$ $=\mathrm{R}\left(J^{\mathrm{c}}\right)$ for all $J \subset A$. In a coupled factorization, the $\mathrm{R}(J)$ form a complete atomic Boolean algebra of factors which is a representation of the complete atomic Boolean algebra of all subsets $J \subset A$. This representation is faithful if $R_{\alpha} \neq 1$ for all $\alpha \in A$.

If $\left\{R_{\alpha}\right\}_{\alpha \in A}$ is a finite type I factorization, then all $\mathrm{R}(J)$ are type I factors and the factorization is coupled..$^{5}$ Thus we have a finite atomic Boolean algebra of type I factors. A complete classification (up to unitary equivalence) of such structures was given by Murray and von Neumann. ${ }^{6}$ )

As mentioned above, lattices of von Neumann algebras occur in recent work in mathematical physics. In the local observables formulation of relativistic quantum field theory, ${ }^{3)}$ one associates a von Neumann algebra $\mathrm{R}(B)$ with every open set $B$ in space-time. A detailed investigation of this lattice for the free scalar field has been given by Araki. ${ }^{4)}$ For this case it is known that $\mathrm{R}(B)$ is a factor of type $\mathrm{III}_{\infty}$ for a certain class of regions $B{ }^{7)}$ It is also known that for a general class of theories, $\mathrm{R}(B)$ is not a factor of type I for a certain class of $B .^{8), 9,10)}$

Similarly, in the formulation of quantum statistical mechanics in an infinite volume one has a representation $B \rightarrow \mathrm{R}(B)$ of the lattice of all regions $B$ of 3-dimensional space by von Neumann algebras $\mathrm{R}(B)$. If one takes $\mathrm{R}(B)$ to be the von Neumann algebra generated by the field operators $\psi(x), x \in B$ where $\psi(x)$ satisfies CCRs for Bose systems and CARs (canonical anticommutation relations) for Fermi systems, then $\mathrm{R}(B)$ is expected to be a factor of type I for any bounded $B .{ }^{11}$ An explicit construction of

6) Lemma 5.4.1 of reference 1 states that any such factorization is a tenslor product factorization. That is $H=\otimes H_{i}$, and $R_{i}=\mathrm{B}\left(H_{i}\right) \otimes \underset{j \neq i}{\left.\otimes \mathbb{1}_{j}\right)}$ where $\mathbb{1}_{j}$ denotes the ring of multiples of the identity operator in $H_{j}$. The unitary invariants are thus the dimensions of the spaces $H_{i}$.

7) H. Araki, Prog. Theor. Phys. 32, 956 (1964).

8) H. Araki, J. Math. Phys. 5, 1 (1964).

9) R.V. Kadison, J. Math. Phys. 4, 1511 (1963).

10) R.F. Streater, J. Math. Phys. 5, 581 (1964).

11) Since one is describing a system with a finite particle density, one requires that the particle number operator $N(B)$ should exist for any bounded region $B$. Thus the representation of the CCRs or CARs restricted to a bounded region $B$ must have a total number operator. It was shown by L. Gårding and A. S. Wightman, Proc. Nat. Acad. Sci. 40, 617, 622 (1954) that any representation of the CCRs or CARs with a total number operator must be a discrete direct sum of copies of the (irreducible) Fock representation. This implies that $\mathrm{R}(B)$ is a factor of type $\mathrm{I}$. 
the representations of the CCRs describing the infinite free Bose gas has been given by Araki and Woods. ${ }^{12}$ The representations of the CARs for the infinite free Fermi gas have been obtained by Araki and Wyss, ${ }^{13)}$ and for the BCS model at zero temperature by Haag, ${ }^{14)}$ and also by Ezawa and by Emch and Guenin. ${ }^{15)}$ While a detailed analysis of the lattice $B \rightarrow \mathrm{R}(B)$ has not been given, in each of these cases some $\mathrm{R}(B)$ are not type I. In particular, for the infinite free Bose gas at finite temperatures, the von Neumann algebra $\mathrm{R}(B)$ where $B$ is the entire 3-dimensional Euclidean space $R^{3}$ is either a factor of type III $_{\infty}$, or a direct integral of such factors.

For such applications to mathematical physics, it would be useful to have a classification of such lattices. In view of the fact that a complete classification theory of non-type I factors does not yet exist, this is a formidable problem. However, our partial classification of complete Boolean algebras of type I factors can be considered as a first step towards the understanding of such structures.

We now give a brief outline of the contents of our paper. In Sec. 2 we discuss finite type I factorizations. In Sec. 3 we discuss the ITPS $H=\bigotimes_{\alpha \in A} H_{\alpha}$ defined by von Neumann. For each $\alpha \in A$, let $R_{\alpha}=\mathrm{B}(H) \otimes$ $\left(\underset{a^{\prime} \neq \alpha}{\left.\otimes \mathbb{1}_{\omega^{\prime}}\right)}\right.$. Then $\{R\}_{\alpha \in A}$ is a type I factorization and all $\mathrm{R}(J)=\bigvee_{\alpha \in I} R_{\omega}$ are type I factors. Thus we have a complete atomic Boolean algebra of type I factors. In Sec. 4 we prove the converse result that any complete atomic Boolean algebra of type I factors is a tensor product factorization (TPF). In Sec. 5 we define an exponential Hilbert space $\mathrm{e}^{K}$ such that any complete Boolean algebra $\mathfrak{P}$ of projections on $K$ gives a complete Boolean algebra $\mathrm{e}^{\mathfrak{B}}$ of type I factors on $\mathrm{e}^{K}$. We define a continuous tensor product of Hilbert spaces (CTPS), which is a generalization of the infinite discrete incomplete tensor product space (ITPS) defined by von Neumann. ${ }^{2)}$ In Sec. 6 we prove that any nonatomic complete Boolean algebra of type I factors $(H, \Re)$

12) H. Araki and E.J. Woods, J. Math. Phys. 4, 637 (1963).

13) H. Araki and W. Wyss, Helv. Phys. Acta 37, 136 (1964).

14) R. Haag, Nuovo Cimento 25, 287 (1962).

15) H. Ezawa, J. Math. Phys. 6, 380 (1965); G. Emch and M. Guenin, J. Math. Phys. (to appear). 
satisfying a certain condition is unitarily equivalent to the structure $\left(\mathrm{e}^{K}, \mathrm{e}^{\mathfrak{B}}\right)$ where $\mathfrak{P}$ is a nonatomic complete Boolean algebra of projections on the Hilbert space $K$. Also $(H, \Re)$ determines $(K, \mathfrak{F})$ up to unitary equivalence. If $K$ is separable, then $\mathfrak{S}$ gives a direct integral decomposition of $K{ }^{16)}$ and $\Re$ is a continuous tensor product factorization. We also determine all factorizable vectors and all factorizable bounded linear operators (relative to $\mathrm{e}^{\mathfrak{\beta}}$ ). In Sec. 7 we establish necessary and sufficient conditions that the group integral for irreducible representations of the CCRs introduced by Klauder and McKenna ${ }^{17), 18}$ exists, and that it is independent of the order of integration. In Sec. 8 we generalize the construction of an exponential space to entire function spaces. In Sec. 9 we give a discussion of our results.

We shall use the following notation. $I_{n}$ denotes the set of integers $(1,2, \cdots, n), I_{\infty}$ the set of all positive integers, and let $I_{n}^{\mathrm{c}}=I_{\infty}-I_{n}$. If $S$ is a subset of the complex numbers, then $S^{(-)}$denotes its closure in the usual topology. If $S$ is a subset of a Hilbert space, then $S^{(w)}, S^{(s)}$ denote the weak and strong closures of $S$ respectively. If $E$ is a lattice with a minimal element 0 , a partition of $z \in E$ is a set of elements $z_{a j}$ such that $z_{a} \wedge z_{\beta}=0$ if $\alpha \neq \beta$, and $\vee_{a} z_{\alpha}=z$. A partition $B=\left\{z_{\alpha j}\right\}$ is a subpartition of $B^{\prime}=\left\{z_{v}^{\prime}\right\}$ if there exists $\nu(\alpha)$ for each $\alpha$ such that $z_{\alpha}<z_{v(\alpha)}^{\prime}$. We then write $B<B^{\prime}$.

\section{Finite Type I Factorizations}

Let $\left\{R_{i}\right\}_{i \in I_{n}}$ be a finite type I factorization (see definition 1.1). It was shown by Murray and von Neumann that any finite type I factorization is unitarily equivalent to the tensor product factorization ${ }^{6)}$

$$
\begin{aligned}
& H=\bigotimes_{i=1}^{n} H_{i} \\
& R_{i}=\mathrm{B}\left(H_{i}\right) \otimes\left({\left.\underset{j \neq i}{\otimes} \mathbf{1}_{j}\right)}\right)
\end{aligned}
$$

16) See, for example, J. Dixmier, Les Algebres d'Operateurs dans l'Espace Hilbertien, (Gauthier- Villars, Paris, 1957), Chap. II, Sec. 6.

17) J.R. Klauder and J. McKenna, J. Math. Phys. 6, 68 (1965).

18) J.R. Klauder, J. McKenna, and E.J. Woods, J. Math. Phys. 7, 822 (1966). 
It follows that the set of all

$$
\mathrm{R}(J)=\bigvee_{i \in I} R_{i}, \quad J \subset A
$$

gives a finite atomic Boolean algebra of type I factors (see Eq. (1. 11) et seq.). In this section we give the relevant properties of such structures.

For $\Psi \in H_{1} \otimes H_{2}$ we define a standard diagonal expansion of $\Psi$. We define factorizable vectors for arbitrary type I factorizations $\left\{R_{a}\right\}_{a \in A}$. For finite type I factorizations $\left\{R_{i}\right\}_{i \in I_{n}}$ we define a quantity $\mathrm{d}\left(\Psi ; R_{1}, \ldots, R\right)$ which is a measure of how close $\Psi$ is to being a product vector. Lemmas 2. 1-2. 5 give some basic properties of $\mathrm{d}\left(\Psi ; R_{1}, \cdots, R_{n}\right)$. Lemma 2.6 proves the equivalence of factorizable vectors and product vectors for finite type I factorizations. The key lemma of this section is lemma 2.7 which relates a finite type I factorization $\left\{R_{i}\right\}_{i \in I_{n}}$ into $n$ factors with the factorizations $\mathrm{R}(J), \mathrm{R}\left(J^{\mathrm{c}}\right)$ into two factors. It will be used in lemmas 4.5 and 4.8.

Let $\Psi \in H$. Then $\left(\Psi, Q_{i} \Psi\right), Q_{i} \in R_{i}$ is a normal state on $R_{i}$. Hence there is a nonnegative trace class operator $\mathrm{D}_{i}(\Psi) \in \mathrm{B}\left(H_{i}\right)$ such that ${ }^{19}$

$$
\operatorname{Tr} \mathrm{D}_{i}(\Psi) Q_{i}=\left(\Psi, Q_{i} \otimes\left(\underset{j \neq i}{\otimes} \mathbb{1}_{j}\right) \Psi\right), \quad Q_{i} \in \mathrm{B}\left(H_{i}\right)
$$

In particular $\operatorname{Tr} \mathrm{D}_{i}(\mathrm{~T})=\|\Psi\|^{2}$. Eq. (2.4) defines $\mathrm{D}_{i}(\Psi)$ uniquely as a normal state over $R_{i}$, and therefore the eigenvalues of $\mathrm{D}_{i}(\Psi)$ and their multiplicities are unitary invariants for $\left(R_{i}, \Psi\right)$.

We now consider the case $n=2$. Let $\Psi_{1 i}$ be an orthonormal basis of $H_{1}$ such that

$$
D_{1}(\Psi) \psi_{1 i}=\lambda_{i}^{\prime} \Psi_{1 i}, \quad \lambda_{i}^{\prime} \geqq 0, \quad \sum_{i} \lambda_{i}^{\prime}<\infty .
$$

Then we can expand $\Psi$ as

$$
\Psi=\Sigma \psi_{1 i} \otimes \phi_{2 i}
$$

For any $Q$ in $\mathrm{B}\left(H_{1}\right)$, we have

$$
(\Psi, Q \Psi)=\Sigma_{i, j}\left(\phi_{2 i}, \phi_{2 j}\right)\left(\psi_{1 i}, Q \psi_{1 j}\right)=\Sigma_{i} \lambda_{i}^{\prime}\left(\psi_{1 i}, Q \psi_{1 i}\right) .
$$

By taking $Q$, such that

19) See J. Dixmier (reference 16), Theorem I. 4.1, p. 54. 


$$
\left(\psi_{1 i}, Q \psi_{1 j}\right)=\delta_{i i_{0}} \delta_{j_{0}}
$$

we have

$$
\left(\phi_{2 i}, \phi_{2 j}\right)=\delta_{i j} \lambda_{i}^{\prime}
$$

Hence

$$
\psi_{2 i}=\left(\lambda_{i}^{\prime}\right)^{-1 / 2} \phi_{2 i} \quad\left(\lambda_{i} \neq 0\right)
$$

is orthonormal and

$$
\Psi=\Sigma_{i}\left(\lambda_{i}^{\prime}\right)^{+1 / 2} \psi_{1 i} \otimes \psi_{2 i}
$$

Thus we make the following

Definition 2. 1: By a standard diagonal expansion of $\Psi \in H_{1} \otimes H_{2}$ (or relative to $R_{1}$ and $R_{2}$ ) we mean a choice of orthonormal bases $\Psi_{1 i} \in H_{1}, \Psi_{2 i} \in H_{2}$ such that

$$
\Psi=\Sigma_{i} \lambda_{i} \Psi_{1 i} \otimes \Psi_{2 i}
$$

where $\lambda_{1} \geqq \lambda_{2} \geqq \cdots \cdots \geqq 0 .{ }^{20}$ ) (If the number of basis vectors can not be taken equal, we allow $\Psi_{1 i}=0$ or $\Psi_{2 i}=0$ when $\lambda_{i}=0$ for the sake of notational convenience.)

Let $P_{1 i}$ be the projection operator onto $\Psi_{1 i}$. Then $\mathrm{D}_{1}(\Psi)$ has the spectral decomposition

$$
\mathrm{D}_{1}(\Psi)=\sum \lambda_{i}^{2} P_{1 i}
$$

We have

$$
\text { Trace } \begin{aligned}
\mathrm{D}_{1}(\Psi)^{2} & =\sum \lambda_{i}^{4}=\lambda_{1}^{2} \sum \lambda_{i}^{2}\left(\lambda_{i} / \lambda_{1}\right)^{2} \\
& \leqslant \lambda_{1}^{2} \sum \lambda_{i}^{2}=\lambda_{1}^{2} \text { Trace } \mathrm{D}_{1}(\Psi) \\
& =\lambda_{1}^{2}\left\|\Psi_{\mid}^{\prime}\right\|^{2}
\end{aligned}
$$

with equality holding if and only if $\lambda_{i}=0, i \neq 1$ (i. e. if $\mathrm{D}_{1}(\Psi)$ is a onedimensional projection operator). We now give some definitions.

\footnotetext{
Definition 2. 2: Let $H=\bigotimes_{i=1}^{n} H_{i} \cdot \quad A$ vector $\Psi \in H$ is called a product vector if
}

20) The standard diagonal expansion of $\psi$ for the case where $H_{1}$ and $H_{2}$ are finite dimensional was given by J. von Neumann (reference 2), Sec. 7.3. 


$$
\Psi=\bigotimes_{i=1}^{n} \Phi_{i}, \quad \Psi_{i} \in H_{i}
$$

Definition 2.3: Let $\left\{R_{\alpha}\right\}_{\omega \in A}$ be a type I factorization ( $A$ is arbitrary). We say that a vector $\Psi$ is factorizable if for all $Q_{\omega} \in R_{a}$ and all integers $n$ we have

$$
\left(\Psi, \prod_{i=1}^{n} Q_{\alpha_{i}} \Psi\right)=(\Psi, \Psi)^{1-n} \prod_{i=1}^{n}\left(\Psi, Q_{\alpha_{i}} \Psi\right)
$$

Definition 2.4: Let $\left\{R_{i}\right\}_{i \in I_{n}}$ be a finite type I factorization. For any $\Psi \in H$ we define

$$
\mathrm{d}\left(\Psi ; R_{1}, \cdots, R_{n}\right)=\sup _{P_{i}}\left(\Psi, \prod_{i=1}^{n} P_{i} \Psi\right)
$$

where $P_{i}$ runs over all minimal projections in $R_{i}{ }^{21)}$

Clearly $\Psi$ is a product vector if and only if there exist minimal projections $P_{i} \in R_{i}$ for each $i$ such that $P_{i} \Psi=\Psi$. It is also obvious that Eq, (2.17) is equivalent to

$$
\left.\mathrm{d}\left(\Psi ; R_{1}, \cdots, R_{n}\right)=\sup _{\Phi_{i}} \mid\left(\Psi, \bigotimes_{i=1}^{n} \Phi_{i}\right)\right)\left.\right|^{2}
$$

where $\Phi_{i}$ runs over all unit vectors in $H_{i}$.

Lemma 2.1: There exist $\otimes \Phi_{i}$ and minimal projections $P_{i}$ which give the suprema in Eqs. (2.17) and (2.18).

Proof: Since the unit sphere in Hilbert space is weakly sequentially compact, there exist weakly convergent sequences $\Phi^{(k)}=\otimes \Phi^{(k)}$ such that

$$
\lim _{k \rightarrow \infty}\left|\left(\Psi, \Phi^{(k)}\right)\right|^{2}=\mathrm{d}\left(\Psi ; R_{1}, \cdots, R_{n}\right)
$$

Let $\Phi=$ weak $\lim \Phi^{(k)}$, then

$$
|(\Psi, \Phi)|^{2}=\mathrm{d}\left(\Psi ; R_{1}, \cdots, R_{n}\right)
$$

21) The quantity $\mathrm{d}\left(\psi ; R_{1}, R_{2}\right)$ was used by H. Araki, J. Math. Phys. 4, 1343 (1963), Theorem 5, to give a condition that a certain factor $R$ obtained by a tensor product construction is type $I$. The same quantity (but in a different notation and for a restricted case) was used by J. von Neumann (reference 2), Sec. 7.3, and D. Bures, Compositio Math. 15, 169 (1963) for a similar purpose. 
We now show that $\Phi$ is a product vector. Since the unit sphere in Hilbert space is weakly sequentially compact, there exists a subsequence of $\Phi_{1}^{(k)}$ with a weak limit $\Phi_{1}$.

This subsequence has a subsubsequence such that $\Phi_{2}^{(k)}$ has a weak limit $\Phi_{2}$, etc. It follows that $\Phi$ is a product vector $\otimes_{i=1}^{n} \Phi_{i}$. Also, from Eq. (2. 20) we must have $\| \Phi_{i} i=1$. Q.E.D.

Lemma 2. 2: Let $\Psi \in H_{1} \otimes H_{2}$. Let $\lambda_{1}$ be the leading term in the standard diagonal expansion of $\Psi$, and $\mathrm{D}_{1}(\Phi)$ the nonnegative trace class operator defined by Eq. (2.4). Then

$$
\mathrm{d}\left(\Psi ; R_{1}, R_{2}\right)=\lambda_{1}^{2} \geq \operatorname{Tr} \mathrm{D}_{1}(\Psi)^{2} / \operatorname{Tr} \mathrm{D}_{1}(\Psi)
$$

Proof: Choose orthonormal bases $\Psi_{1 i}, \Psi_{2 i}$ for $H_{1}, H_{2}$ respectively which give the standard diagonal expansion of $\Psi$ (Eq. (2.12)). Let

$$
\begin{array}{ll}
\Phi_{1}=\sum \alpha_{i} \Psi_{1 i}, & \sum\left|\alpha_{i}\right|^{2}=1 \\
\Phi_{2}=\sum \beta_{i} \Psi_{2 i}, & \sum\left|\beta_{i}\right|^{2}=1
\end{array}
$$

be any two unit vectors in $H_{1}, H_{2}$ respectively. Then

$$
\left|\left(\Psi, \Phi_{1} \otimes \Phi_{2}\right)\right|^{2}=\left|\sum \lambda_{i} \alpha_{i} \beta_{i}\right|^{2} \leqq\left(\sum \lambda_{i}^{2} \mid \alpha_{i} !^{\prime 2}\right) \quad\left(\sum\left|\beta_{i}\right|^{2}\right)
$$

Since $\lambda_{1} \geq \lambda_{2} \geq \cdots \geq 0$ and $\Sigma\left|\alpha_{i}\right|^{2}=\sum\left|\beta_{i}\right|^{2}=1$, this has its maximum value $\lambda_{1}^{2}$ when $\Phi_{1}=\Psi_{11}, \Phi_{2}=\Psi_{21}$. The inequality in Eq. (2.21) follows from Eq. (2. 14). Q.E.D.

Lemma 2.3: Let $\left\{R_{i}\right\}_{i \in I_{n}}$ be a finite type I factorization. For any $j \in I_{n}$ we have

$$
\mathrm{d}\left(\Psi ; R_{1} \cdots R_{n}\right)=\sup _{P_{i}, i \neq j}\left(\Psi, \prod_{i \neq j} P_{i} \Psi\right)
$$

where $P_{i}$ runs over all minimal projections in $P_{i}, i \neq j$.

Proof: It suffices to show that for any minimal $P_{i}, i \neq j$ there is some minimal $P_{j}$ such that

$$
P_{j}\left(\prod_{i \neq j} P_{i} \Psi\right)=\prod_{i \neq j} P_{i} \Psi
$$

Choose orthonormal bases for each $H_{i}$ such that for $i \neq i, P_{i}$ is the minimal projection associated with $\Psi_{i_{0}}$. Then 


$$
\prod_{i \neq j} P_{i} \Psi=\sum C_{\infty} \Psi_{j \omega} \otimes\left\{\bigotimes_{i \neq j} \Psi_{i 0}\right\}
$$

is a product vector. The minimal projection associated with the vector $\sum C_{a} \Psi_{j a}$ is the desired $P_{j}$. Q.E.D.

Lemma 2. 4: Let $\left\{R_{i}\right\}_{i \in I_{n}}$ be a finite type I factorization. Let $\Phi, \Psi \in H,\|\Phi-\Psi\|<\varepsilon$. Then

$$
\left|\mathrm{d}\left(\Psi ; R_{1}, \cdots, R_{n}\right)-\mathrm{d}\left(\Phi ; R_{1}, \cdots, R_{n}\right)\right| \leq(\|\Psi\|+\|\Phi\|) \varepsilon
$$

Proof: By Eq. (2. 18) it suffices to consider

$$
\begin{aligned}
& \left.||\left(\Psi, \otimes \Phi_{i}\right)\right|^{2}-\left|\left(\Phi, \otimes \Phi_{i}\right)\right|^{2} \mid \\
& =\left\{\left|\left(\Psi, \otimes \Phi_{i}\right)+\right|\left(\Phi, \otimes \Phi_{i}\right) \mid\right\}\left\{||\left(\Psi, \otimes \Phi_{i}\right)|-|\left(\Phi, \otimes \Phi_{i}\right) \|\right\} \\
& \leq(\|\Psi\|+\|\Phi\|) \varepsilon
\end{aligned}
$$

since $\left\|\otimes \Phi_{i}\right\|=1$. Q.E.D.

Lemma 2. 5: Given $\Psi \in H_{1} \otimes H_{2},\|\Psi\|=1$. Let

$$
\rho=\sup _{Q_{1}, Q_{2}}\left|\left(\Psi, Q_{1}, Q_{2} \Psi\right)-\left(\Psi, Q_{1} \Psi\right)\left(\Psi, Q_{2} \Psi\right)\right| /\left(\left\|Q_{1}\right\| \cdot\left\|Q_{2}\right\|\right)
$$

where $Q_{1}, Q_{2}$ run over all operators in $R_{1}, R_{2}$ respectively. If $\rho<4 / 25$ we have

$$
1-\mathrm{d}\left(\Psi ; R_{1}, R_{2}\right) \leq \frac{1}{2}\left[1-(1-4 \rho)^{1 / 2}\right]
$$

Proof: Let

$$
\Psi=\sum \lambda_{i} \Psi_{1 i} \otimes \Psi_{2 i}
$$

be a standard diagonal expansion of $\Psi$. By lemma 2.2 we have $\mathrm{d}\left(\Psi ; R_{1}, R_{2}\right)=\lambda_{1}^{2}$. Let $P_{1 i}, P_{2 i}$ be the minimal projections corresponding to $\Psi_{1 i}, \Psi_{2 i}$ respectively. Then we have

$$
\rho \geq\left|\left(\Psi, P_{11} P_{21} \Psi\right)-\left(\Psi, P_{11} \Psi\right)\left(\Psi, P_{21} \Psi\right)\right|=\left|\lambda_{1}^{2}-\lambda_{1}^{4}\right|
$$

which implies either

$$
\lambda_{1}^{2} \geq \frac{1}{2}\left[1+(1-4 \rho)^{1 / 2}\right]
$$

or

$$
\lambda_{1}^{2} \leq \frac{1}{2}\left[1-(1-4 \rho)^{1 / 2}\right]
$$


Assume Eq. (2. 35) is true. We hav $\sum \lambda_{i}^{2}=1$ and $\lambda_{i} \leq \lambda_{1}$. For $\rho<4 / 25$ we have

$$
\sqrt{\rho}+\sqrt{\rho}+\frac{1}{2}\left[1-(1-4 \rho)^{1 / 2}\right]<1
$$

It follows that we can choose $n$ so that

$$
\sum_{i=1}^{n} \lambda_{1}^{2}>\sqrt{\rho} \text { and } \sum_{i>n} \lambda_{i}^{2}>\sqrt{\rho}
$$

Let $Q_{1}=\left(\sum_{i=1}^{n} P_{1 i}\right) \otimes 1, Q_{2}=1 \otimes\left(\sum_{i>n} P_{2 i}\right)$. Then

$$
\rho \geq\left|\left(\Psi, Q_{1} Q_{2} \Psi\right)-\left(\Psi, Q_{1} \Psi\right)\left(\Psi, Q_{2} \Psi\right)\right|>\rho
$$

a contradiction. Q.E.D.

Lemma 2. 6: Let $\left\{R_{a}\right\}_{a \in A}$ be a type I factorization. A vector $\Psi \in H$ is factorizable if and only if there exist minimal projections $P_{a} \in R_{\infty}$ for each $\alpha \in A$ such that $P_{\alpha} \Psi=\Psi$. If the set $A$ is finite, then $\Psi$ is factorizable if and only if it is a product vector.

Proof: We can assume $\|\Psi\|=1$. We first show that the existence of minimal $P_{\alpha}$ with $P_{\alpha} \Psi=\Psi$ implies that $\Psi$ is factorizable. Thus let $\alpha_{1}, \cdots, \alpha_{n}$ be given. Then we have

$$
\begin{aligned}
& H=\left(\bigotimes_{i=1}^{n} H_{\alpha_{i}}\right) \otimes H^{\prime} \\
& R_{\alpha_{i}}=\left\{B\left(H_{\alpha_{i}}\right) \otimes\left(\bigotimes_{\substack{j=1 \\
j \neq i}}^{n} \mathbb{1}_{j}\right)\right\} \otimes \mathbb{1}
\end{aligned}
$$

But $\prod_{i=1}^{n} P_{\alpha_{i}} \Psi$ is a product vector with respect to this decomposition. It follows that

$$
\left(\Psi, \prod_{i=1}^{n} Q_{\alpha_{i}} \Psi\right)=\prod_{i=1}^{n}\left(\Psi, Q_{\alpha_{i}} \Psi\right)
$$

for all $Q_{\alpha_{i}} \in R_{\alpha_{i}}$. Thus $\Psi$ is factorizable.

We now prove the converse. For each $\alpha \in A$ we have

$$
\begin{aligned}
& H=H_{a} \otimes H_{\alpha}^{\prime} \\
& R_{\infty}=\mathrm{B}\left(H_{\beta}\right) \otimes \mathbb{1}
\end{aligned}
$$


For all $Q_{\beta} \in R_{\beta}$ and all $n$ we have

$$
\left(\Psi, Q_{a_{i=1}} \prod_{\alpha_{i}}^{n} \Psi\right)=\left(\Psi, Q_{\infty} \Psi\right)\left(\Psi, \prod_{i=1}^{n} Q_{\alpha_{i}} \Psi\right)
$$

Since the union of $R_{\beta}, \beta \neq \alpha$ is irreducible on $H_{\alpha}^{\prime}$, any $Q^{\prime} \in 1 \otimes \mathbf{B}\left(H_{\alpha}^{\prime}\right)$ can be approximated weakly by operators of the form

$$
\sum_{j=1}^{m} C_{j}\left(\left\{\alpha_{i}\right\}\right) \prod_{i=1}^{n_{j}} Q_{\alpha_{i}}
$$

It follows that

$$
\left(\Psi, Q_{a} Q^{\prime} \Psi\right)=\left(\Psi, Q_{a} \Psi\right)\left(\Psi, Q^{\prime} \Psi\right)
$$

for all $Q_{\omega} \in R_{a}, Q^{\prime} \in R_{\alpha}^{\prime}$. It now follows from the preceding lemma that $\Psi$ is a product vector with respect to the decomposition $H_{\alpha}=H_{\infty} \otimes H_{\alpha}^{\prime}$. Thus there exists a minimal projection $P_{a} \in R_{a}$ such that $P_{a} \Psi=\Psi$.

If the set $A$ is finite, then we can tacke $A=I_{n}$ and we have $H=\bigotimes_{i=1}^{n} H_{i}$. Clearly $\Psi=\prod_{i=1}^{n} P_{i} \Psi$ is a product vector. Q.E.D.

Lemma 2. 7: Let $\left\{R_{i}\right\}_{i \in I_{n}}$ be a finite type I factorization., For any unit vector $\Psi \in H$ let

$$
\begin{aligned}
& d_{1}=\mathrm{d}\left(\Psi ; R_{1}, \cdots, R_{n}\right) \\
& d_{2}=\inf \mathrm{d}\left(\Psi ; R(J), R\left(J^{\mathrm{c}}\right)\right)
\end{aligned}
$$

where $J$ runs over all subsets of $I_{n}$. Then there exist positive numbers $\varepsilon$ and $\delta$ such that

$$
1-d_{2}<\varepsilon
$$

implies

$$
\left(1-d_{2} \leq\right) 1-d_{1}<\delta\left(1-d_{2}\right)
$$

Proof: Our proof consists of three parts. In the first part we obtain a subset $J$ satisfying a certain condition (Eq. (2.57)). Choose a product vector $\otimes \Phi_{i} \in H$ such that

$$
\left|\left(\Psi, \otimes \Phi_{i}\right)\right|^{2}=d_{1}
$$

For each $j \in I_{n}$ let $A_{j}$ be an index set containing 0 such that the cardinal of $A_{j}$ is $\operatorname{dim} H_{j}$. Let $\Psi_{j k}, k \in A_{j}$ be an orthonormal basis for $H_{j}$ such 
that $\Psi_{j 0}=\Phi_{j}$. Let $\alpha$ denote the sequence $\left(\alpha_{1}, \cdots, \alpha_{n}\right), \alpha_{j} \in A_{j} . \quad \alpha=0$ denotes the sequence with all $\alpha_{j}=0$. The vecotrs

$$
\Psi(\alpha)=\bigotimes_{j=1}^{n} \Psi_{j^{\prime} j}
$$

form an orthonormal basis for $H$. We now expand

$$
\Psi=\sum_{a} \mathrm{C}(\alpha) \Psi(\alpha)
$$

Then

$$
d_{1}=|\mathrm{C}(0)|^{2} \quad \text { and } \quad 1-d_{1}=\sum_{\alpha \neq 0}|\mathrm{C}(\alpha)|^{2}
$$

We note that if $\mathbf{C}(\alpha) \neq 0$ then at least two $\alpha_{j} \neq 0$, otherwise one could construct a vector $\otimes \chi_{i}$ with $\left|\left(\Psi, \otimes \chi_{1}\right)\right|^{2}>d_{1}$. Let $J \cdot J^{\mathrm{c}}$ be some partition of $I_{n}$. This induces a splitting of the sequence $\alpha$ which we write $\alpha=\left\{\alpha(J), \alpha\left(J^{\mathrm{c}}\right)\right\}$. Let

$$
\begin{aligned}
& \mathrm{A}(J)=\left\{\alpha: \alpha(J) \neq 0, \alpha\left(J^{\mathrm{c}}\right)=0\right\} \\
& \mathrm{A}\left(J, J^{\mathrm{c}}\right)=\left\{\alpha: \alpha(J) \neq 0, \alpha\left(J^{\mathrm{c}}\right) \neq 0\right\} \\
& \mathrm{C}(J)=\sum_{\alpha \in \mathrm{A}(J)}|\mathrm{C}(\alpha)|^{2} \\
& \mathrm{C}\left(J, J^{\mathrm{c}}\right)=\sum_{\alpha \in \mathrm{A}\left(J, J^{n}\right)}|\mathrm{C}(\alpha)|^{2}
\end{aligned}
$$

We will prove that there exists a $J$ such that

$$
\mathrm{C}(J)+\mathrm{C}\left(J^{\mathrm{c}}\right) \leq \frac{1}{2}\left(1-d_{1}\right)
$$

Let $\mathrm{N}(\alpha)$ be the number of $\alpha_{j} \neq 0$. Let

$$
\begin{aligned}
& \mathrm{k}(J)=\sum_{\alpha \in \mathrm{A}\left(J, J^{c}\right)}|\mathrm{C}(\alpha)|^{2} / \mathrm{N}(\alpha) \\
& \mathrm{A}(j)=\left\{\alpha: \alpha_{j} \neq 0\right\} \\
& \mathrm{A}_{1}(j, J)=\mathrm{A}(j) \cap \mathrm{A}\left(J, J^{\mathrm{c}}\right) \\
& \mathrm{A}_{2}(j, J)=\left\{\begin{array}{lr}
\mathrm{A}(j) \cap \mathrm{A}\left(J^{\mathrm{c}}\right) & \text { if } j \in J^{\mathrm{c}} \\
\mathrm{A}(j) \cap \mathrm{A}(J) & \text { if } j \in J
\end{array}\right. \\
& \mathrm{k}_{i}(j, J)=\sum_{\alpha \in \mathrm{A}_{i}(j, J)}|\mathrm{C}(\alpha)|^{2} / \mathrm{N}(\alpha), \quad i=1,2
\end{aligned}
$$

Choose some $J$ for which $\mathrm{k}(J)$ is maximal. For any $j \in J$, let $J^{\prime}=J-\{j\}$. 
Since $\mathrm{N}(\alpha)=1$ implies $\mathrm{C}(\alpha)=0$, we have

$$
\mathrm{k}\left(J^{\prime}\right)-\mathrm{k}(J) \geq \mathrm{k}_{2}(j, J)-\mathrm{k}_{1}(j, J)
$$

We obtain the same equation for $j \in J^{\mathrm{c}}$ by a similar argument with $J^{\prime}=J \cup\{j\}$. Thus $\mathrm{k}(J)$ maximal implies that $\mathrm{k}_{2}(j, J) \leq \mathrm{k}_{1}(j, J)$. Now

$$
\mathrm{C}\left(J, J^{\mathrm{c}}\right)=\Sigma_{j} \mathrm{k}_{1}(j, J) \geq \Sigma_{j} \mathrm{k}_{2}(j, J)=\mathrm{C}(J)+\mathrm{C}\left(J^{\mathrm{c}}\right)
$$

Since

$$
\mathrm{C}(J)+\mathrm{C}\left(J^{\mathrm{c}}\right)+\mathrm{C}\left(J, J^{\mathrm{c}}\right)=1-d_{1}
$$

we have

$$
\mathrm{C}(J)+\mathrm{C}\left(J^{\mathrm{c}}\right) \leq \frac{1}{2}\left(1-d_{1}\right)
$$

This completes the first part of the proof.

We now prove that there exist positive numbers $\varepsilon_{1}$ and $\delta$ such that

$$
1-d_{1} \leq \varepsilon_{1} \text { implies } 1-d_{1} \leq \delta\left(1-d_{2}\right)
$$

Write $H=\mathrm{H}(J) \otimes \mathrm{H}\left(J^{\mathrm{c}}\right)$. We have

$$
\Psi(\alpha)=\Psi(\alpha(J)) \otimes \Psi\left(\alpha\left(J^{\mathrm{c}}\right)\right)
$$

To simplify notation, let $\alpha(J) \rightarrow \beta, \alpha\left(J^{\mathrm{c}}\right) \rightarrow \gamma$. Then Eq. (2.51) becomes

$$
\Psi=\sum C_{\beta \gamma} \Psi_{\beta}(J) \otimes \Psi_{\gamma}\left(J^{\mathrm{c}}\right)
$$

The quantity

$$
d=\mathrm{d}\left(\Psi ; \mathrm{R}(J), \mathrm{R}\left(J^{\mathrm{c}}\right)\right)
$$

is the largest eigenvalue of $D=C C^{*}$ where $C$ is the coefficient matrix in Eq. (2. 69). Since

$$
1-d_{2} \geq 1-d \geq \frac{1}{2}\left(1-\mathrm{d}^{2}\right) \geq \frac{1}{2}\left(1-\text { Trace } D^{2}\right)
$$

it suffices to find $\varepsilon_{1}, \delta$ such that $1-d_{1} \leq \varepsilon_{1}$ implies

$$
1-\operatorname{Tr} D^{2} \geq 2\left(1-d_{1}\right) / \delta
$$

We write $C=A+B$ where

$$
A_{\beta \gamma}=C_{\beta 0} C_{0 \gamma} / C_{00}
$$


Note that $B_{\beta_{0}}=B_{0 \gamma}=0$. This gives $\Psi=\Psi_{a}+\Psi_{b}$ where

$$
\Psi_{a}=\left[\Sigma_{\beta} C_{\beta_{0}} \Psi_{\beta}(J)\right] \otimes\left[\Sigma_{\gamma} \mathrm{C}_{0 \gamma} \Psi_{\gamma}\left(J^{\mathrm{c}}\right)\right] / C_{00}
$$

We define the unit vector

$$
\Psi^{\prime}(J)=\Sigma_{\beta} C_{\beta_{0}} \Psi_{\beta}(J) /\left[d_{1}+C(J)\right]^{1 / 2}
$$

Let $P$ be the one-dimensional projection onto $\Psi^{\prime}(J)$, and let $d_{a}=\left\|\Psi_{a}\right\|^{2}$. Then we have $A A^{*}=d_{a} P$ and we get

$$
D=d_{a} P+K
$$

where $K=C B^{*}+B A^{*}$. Thus

$$
\operatorname{Tr} D^{2}=d_{a}^{2}+2 d_{a} \operatorname{Tr} P K+\operatorname{Tr} K^{2}
$$

We have

$$
d_{a}=\left\|\Psi_{a}\right\|^{2}=\sum\left|\mathrm{A}_{\beta \gamma}\right|^{2}
$$

Using Eqs. (2.66) and (2.73) we get

$$
\begin{aligned}
d_{a} & =d_{1}+\mathrm{C}(J)+\mathrm{C}\left(J^{\mathrm{c}}\right)+\mathrm{C}(J) \mathrm{C}\left(J^{\mathrm{c}}\right) / d_{1} \\
& \leq d_{1}+\frac{1}{2}\left(1-d_{1}\right)+\left(1-d_{1}\right)^{2} /\left(16 d_{1}\right)
\end{aligned}
$$

Thus

$$
d_{a} \leq \frac{1}{2}\left(1+d_{1}\right)+\left(1-d_{1}\right)^{2} /\left(16 d_{1}\right)
$$

This gives

$$
d_{a}^{2} \leq d_{1}+\left(1-d_{1}\right)^{2} / 4+\left(1-d_{1}\right)^{2}\left(1+d_{1}\right) /\left(16 d_{1}\right)+\left(1-d_{1}\right)^{4} /\left(16 d_{1}\right)^{2}
$$

Then $\left(1-d_{1}\right) \leq \varepsilon_{1}$ for any $\varepsilon_{1}<\frac{1}{2}$ gives

$$
d_{a}^{2} \leq d_{1}+\left(1-d_{1}\right)^{2} / 2
$$

We now introduce the quantities

$$
\begin{aligned}
& a_{\beta}^{2}=\sum_{\gamma \neq 0}\left|A_{\beta \gamma}\right|^{2} \\
& b_{\beta}^{2}=\sum\left|B_{\beta \gamma}\right|^{2} \\
& c_{\beta}^{2}=\sum_{\gamma \neq 0}\left|C_{\beta \gamma}\right|^{2}
\end{aligned}
$$

We have 


$$
\sum c_{\beta}^{2}=\sum_{\beta} \sum_{\gamma \neq 0}\left|C_{\beta \gamma}\right|^{2}=1-d_{1}-\mathrm{C}(J) \leq 1-d_{1}
$$

Since $A_{\beta \gamma}=C_{\beta_{0}} C_{0 \gamma} / C_{00}$ we get

$$
\sum a_{\beta}^{2}=\sum_{\beta} \sum_{\gamma \neq 0}\left|A_{\beta \gamma}\right|^{2}=\left[\mathrm{C}(J)+d_{1}\right]\left[\mathrm{C}\left(J^{\mathrm{c}}\right)\right] / d_{1}
$$

It follows from Eq. (2.66) that

$$
\sum a_{\beta}^{2} \leq \frac{1}{2}\left(1-d_{1}\right)
$$

Since $B_{\beta \gamma}=C_{\beta \gamma}-A_{\beta \gamma}$ we have

$$
\left|B_{\beta \gamma}\right|^{2} \leq 2\left(\left|C_{\beta \gamma}\right|^{2}+\left|A_{\beta \gamma}\right|^{2}\right)
$$

Since $B_{\beta_{0}}=B_{0 \gamma}=0$ it follows that

$$
\begin{aligned}
\sum b_{\beta}^{2} \leq 2 \sum_{\beta \neq 0} \sum_{\gamma \neq 0}\left(\left|C_{\beta \gamma}\right|^{2}+\left|A_{\beta \gamma}\right|^{2}\right) \\
\leq 2\left[1-d_{1}-\mathrm{C}(J)-\mathrm{C}\left(J_{\mathrm{c}}\right)\right]+2 \mathrm{C}(J) \mathrm{C}\left(J^{\mathrm{c}}\right) / d_{1}
\end{aligned}
$$

Then $\left(1-d_{1}\right) \leq \varepsilon_{1}$ for any $\varepsilon_{1}<\frac{1}{2}$ gives

$$
\mathrm{C}(J)+\mathrm{C}\left(J^{\mathrm{c}}\right)-C(J) C\left(J^{\mathrm{c}}\right) / d_{1} \geq 0
$$

Thus

$$
\sum b_{\beta}^{2} \leq 2\left(1-d_{1}\right)
$$

We have

$$
\operatorname{Tr} P K=\left(\Psi^{\prime}(J), K \Psi^{\prime}(J)\right)
$$

Noting that $B_{\beta_{0}}=B_{0 \beta}=0$ we find

$$
\begin{aligned}
& {\left[d_{1}+\mathrm{C}(J)\right]|\operatorname{Tr} P K|=\left|\sum_{\beta, \beta^{\prime}, \gamma} \bar{C}_{\beta_{0}}\left(C_{\beta \gamma} \bar{B}_{\beta^{\prime} \gamma}+B_{\beta \gamma} \bar{A}_{\beta^{\prime} \gamma}\right) C_{\beta^{\prime} 0}\right|} \\
& \quad \leq \sum_{\beta} \sum_{\beta^{\prime} \neq 0}\left|\bar{C}_{\beta 0} c_{\beta} b_{\beta^{\prime}} C_{\beta^{\prime} 0}\right|+\sum_{\beta \neq 0} \sum_{\beta^{\prime}}\left|\bar{C}_{\beta_{0}} b_{\beta} a_{\beta^{\prime}} C_{\beta^{\prime} 0}\right| \\
& \quad \leq\left(1-d_{1}\right)\left\{\mathrm{C}(J)\left[\mathrm{C}(J)+d_{1}\right]\right\}^{1 / 2}(\sqrt{2}+1)
\end{aligned}
$$

Thus

$$
|\operatorname{Tr} P K| \leq\left(1-d_{1}\right)(\sqrt{2}+1)\left\{\mathrm{C}(J) /\left[\mathrm{C}(J)+d_{1}\right]\right\}^{1 / 2}
$$

It remains to estimate $\left|\operatorname{Tr} K^{2}\right|$. We have 


$$
K^{2}=C B^{*} C B^{*}+C B^{*} B A^{*}+B A^{*} C B^{*}+B A^{*} B A^{*}
$$

By a similar calculation to Eq. (2.94) we find

$$
\begin{aligned}
\left|\operatorname{Tr} K^{2}\right| & \leq\left|\sum_{\beta \neq 0} \sum_{\beta^{\prime} \neq 0} c_{\beta} b_{\beta^{\prime}} c_{\beta^{\prime}} b_{\beta}\right|+2\left|\sum_{\beta} \sum_{\beta^{\prime} \neq 0} c_{\beta} b_{\beta^{\prime}}^{2} a_{\beta}\right| \\
& +\left|\sum_{\beta \neq 0} \sum_{\beta^{\prime} \neq 0} b_{\beta} a_{\beta^{\prime}} b_{\beta^{\prime}} a_{\beta}\right| \\
& \leq\left(1-d_{1}\right)^{2}(2+2 \sqrt{2}+1)
\end{aligned}
$$

Then Eqs. (2.77), (2.82), (2.95), (2.97) give

$$
\operatorname{Tr} D^{2} \leq d_{1}+\left(1-d_{1}\right) \mathrm{f}\left(d_{1}\right)
$$

where

$$
\mathrm{f}\left(d_{1}\right)=(7+4 \sqrt{2})\left(1-d_{1}\right) / 2+2(1+\sqrt{2})\left[\left(1-d_{1}\right) /\left(1+d_{1}\right)\right]^{1 / 2}
$$

for $d_{1} \geq \frac{1}{2}$. From Eqs. (2. 71-72) we have

$$
1-d_{2} \geq \frac{1}{2}\left(1-d_{1}\right)\left[1-\mathrm{f}\left(d_{1}\right)\right]
$$

Thus we can choose any $\varepsilon_{1} \leq \frac{1}{2}$ such that

$$
f\left(1-\varepsilon_{1}\right)<1
$$

(which is not satisfied for any $\varepsilon_{1}>\frac{1}{2}$ anyway). Then Eq. (2.67) is satisfied with

$$
\delta \geq 2 /\left[1-\mathrm{f}\left(1-\varepsilon_{1}\right)\right]
$$

In particular $\varepsilon_{1}=1 / 10, \delta=20$ satisfy Eq. (2. 67).

The third and final part of our proof is to show that Eq. (2.67) can be replaced by the condition

$$
1-d_{2} \leq \varepsilon_{1} /(1+\delta) \text { implies } 1-d_{1} \leq \delta\left(1-d_{2}\right)
$$

The proof is by induction on $n$. For $n=2$, we have $d_{1}=d_{2}$ and the result holds trivially. Now assume the result for $n-1$, and consider the factorization $R_{1}, \cdots, R_{n-2}, R_{n-1} \vee R_{n}$ into $n-1$ factors. Let $d_{1}^{\prime}, d_{2}^{\prime}$ denote the quantities $d_{1}, d_{2}$ for this factorization. Let $P_{i} \in R_{i}, i \in I_{n-2}$ be minimal projections such that

$$
\left(\Psi, P_{1} \cdots P_{n-2} \Psi\right)=d_{1}^{\prime}
$$


By assumption we have $1-d_{1}^{\prime} \leq \delta\left(1-d_{2}^{\prime}\right)$. Since $d_{2}^{\prime} \geq d_{2}$ we get

$$
1-d_{1}^{\prime} \leq \delta\left(1-d_{2}\right) \leq \varepsilon_{1} \delta /(1+\delta)
$$

Let $P_{n-1} \in R_{n-1}$ be a minimal projection such that

$$
\left(\Psi, P_{n-1} \Psi\right) \geq d_{2}
$$

Then $\left\|P_{n-1} \Psi-\Psi\right\|^{2} \leq 1-d_{2}$ and

$$
\left\|P_{1} \cdots P_{n-2}\left(P_{n-1} \Psi-\Psi\right)\right\|^{2} \leq 1-d_{2}
$$

If follows that

$$
\begin{aligned}
1-\left(\Psi, P_{1} \cdots P_{n-1} \Psi\right) & \leq\left(1-d_{2}\right)+\left(1-d_{1}^{\prime}\right) \\
& \leq \varepsilon_{1} /(1+\delta)+\varepsilon_{1} \delta /(1+\delta)=\varepsilon_{1}
\end{aligned}
$$

It now follows from Lemma 2.3 that $1-d_{1} \leq \varepsilon_{1}$. Eq. (2.67) then gives

$$
1-d_{1} \leq \delta\left(1-d_{2}\right)
$$

It follows that the positive numbers $\varepsilon=\varepsilon_{1} /(1+\delta)$ and $\delta$ have the desired property. Q.E.D.

Apart from the condition that Eq. (2.101) be satisfied, there is a good deal of freedom in the choice of $\varepsilon, \delta$ in lemma 2.7. The choice $\varepsilon_{1}=1 / 10, \delta=20$, and $\varepsilon=1 / 210$ is one possibility. One can obtain a smaller $\delta$ by choosing a smaller $\varepsilon_{1}$.

\section{Discrete Tensor Products}

In this section we discuss the relevant properties of the incomplete tensor product space (hereafter referred to as ITPS) defined by von Neumann. ${ }^{2)}$ We assume the reader to have some familiarity with the properties of an ITPS. We define an ITPS and discuss product vectors. Lemma 3.1 gives an asymptotic property of vectors in an ITPS. We discuss the associative law, and use it to show that any ITPS gives a complete atomic Boolean algebra of type I factors. Lemma 3. 2 proves that a vector in an ITPS is factorizable if and only if it is a product vector. Lemma 3. 3 proves that a weak limit of product vectors is a product vector.

Let $\left\{H_{\alpha}\right\}_{\alpha \in A}$ be a set of Hilbert spaces, and let $\phi_{\omega} \in H_{\omega}$ be a set of unit vectors. The ITPS 


$$
H=\bigotimes_{\alpha \in A}^{\otimes\left(\otimes \phi^{\infty}\right)} H_{\infty}
$$

containing the product vector $\phi=\otimes \phi_{\omega}$ may be defined as follows. Let $\Psi_{\beta}^{(\alpha)}$ be an orthonormal basis for $H_{\omega}$ such that

$$
\Psi_{0}^{(\alpha)}=\phi_{\omega}
$$

Then an orthonormal basis for the ITPS $H$ is given by the product vectors

$$
\Psi(\beta)=\bigotimes_{\alpha \in A} \Psi_{\beta_{a}}^{(\alpha)}
$$

where $\beta$ runs over all sequences $\beta_{\alpha}, \alpha \in A$ such that $\beta_{\alpha}=0$ a.a. ${ }^{22}$ The ITPS $H$ depends on the reference vector $\otimes \phi_{a}$ (unless the set $A$ is finite). If there is no danger of ambiguity we may write

$$
H=\otimes_{\alpha \in A} H_{\infty}
$$

without explicitly including the reference vector. Note that the product vectors form a total set for $H$. For a subset $J \subset A$, we write $\mathrm{H}(J)=\bigotimes_{\alpha \in J} H_{a}$.

We now discuss briefly the basic properties of product vectors, $\otimes \chi_{a}$, $\chi_{a} \in H_{a}$ where

$$
0<\prod_{\alpha \in A}\left\|\chi_{\infty}\right\|<\infty
$$

Two such product vectors are said to be equivalent, $\otimes \chi_{a} \approx \otimes \psi_{\infty}$ if and only if

$$
\sum_{a \in A}\left|\left(\chi_{\infty}, \psi_{\omega}\right)-1\right|<\infty
$$

This is equivalent to requiring that

$$
\prod_{\alpha \in A}\left(\chi_{a}, \psi_{\infty}\right)
$$

converge. $^{23)}$ Furthermore $\left(\chi_{a}, \psi_{a}\right) \neq 0$ for all $\alpha$ implies that

$$
\prod_{\alpha \in A}\left(\chi_{\alpha}, \psi_{a}\right) \neq 0
$$

22) $\beta_{\alpha}=0$ a.a. means $\beta_{\alpha}=0$ except for a finite number of $\alpha$. The a.a. reads "almost always".

23) If $z_{\alpha}$ are complex numbers, then $\Pi_{z_{\alpha}}$ converges if and only if $\sum|1-z|<\infty$. If $\Pi_{z_{a}}$ converges and $z_{\alpha} \neq 0$ for all $\alpha$, then $\Pi_{z_{\alpha}} \neq 0$. Cf. J. von Neumann (reference 2), lemma 2.4.1. 
If $\otimes \chi_{a} \approx \otimes \phi_{a}$, we may define it as a vector in the ITPS $H$ determined by the reference vector $\otimes \phi_{\infty}$ as follows. We have

$$
\chi_{a}=\sum_{\beta_{\alpha}} \mathrm{C}_{a}\left(\beta_{a}\right) \Psi_{\beta_{\alpha}}^{(\alpha)}
$$

For any finite subset $J \subset A$ let

$$
\begin{aligned}
\chi(J) & =\left\{\underset{\alpha \in J}{\otimes} \chi_{\alpha}\right\} \otimes\left\{\bigotimes_{\alpha \in J^{c}} \Psi_{0}^{(\alpha)}\right\} \\
& =\sum \mathrm{C}_{J}(\beta) \Psi(\beta)
\end{aligned}
$$

where

$$
\mathrm{C}_{J}(\beta)=\left\{\prod_{\alpha \in J} \mathrm{C}_{\alpha}\left(\beta_{\alpha}\right)\right\}\left\{\underset{\alpha \in J}{\mathrm{c}} \delta_{\beta_{\alpha}, 0}\right\}
$$

Eq. (3. 6) now implies that

$$
\lim _{J \uparrow} \chi(J) \equiv \bigcap_{J}\left\{\bigcup_{J^{\prime} \supset J} \chi\left(J^{\prime}\right)\right\}^{(\mathrm{s})}
$$

exists (where $J^{\prime}$ is finite, and \{\}$^{(\mathrm{s})}$ denotes the strong closure of the set \{\}$)$. We shall have occasion to make use of such limits in the following. Conversely, if $\chi$ is a product vector $\otimes \chi_{\omega} \in H$, then

$$
\prod_{\alpha \in A}\left(\chi_{a}, \phi_{a}\right)=(\chi, \phi)
$$

and. $\otimes \chi_{a} \approx \otimes \phi_{a}$.

Lemma 3. 1: Let $\Psi$ be a vector in the ITPS $H=\bigotimes_{\alpha \in A}^{\left(\otimes r^{\prime}\right)} H_{a}$. Given $\varepsilon>0$, there exists a finite $J \subset A$ and $\Psi_{J} \in \mathrm{H}(J)$ such that

$$
\left\|\Psi-\Psi_{J} \otimes\left\{\bigotimes_{\alpha \in J^{c}} \phi_{\infty}\right\}\right\|<\varepsilon
$$

Proof: Choose basis vectors $\Psi(\beta)$ as in Eqs. (3.1-3). Then we can expand

$$
\Psi=\Sigma_{\beta} \mathrm{C}(\beta) \Psi(\beta)
$$

Choose a finite subset $B$ of the $\beta$ so that

$$
\sum_{\beta \in B^{\mathrm{c}}}|\mathrm{C}(\beta)|^{2}<\varepsilon^{2}
$$

Let $J^{\mathrm{c}}=\left\{\alpha: \beta_{a}=0\right.$ for all $\left.\beta \in B\right\}$. Then $J$ is a finite set and

$$
\sum_{\beta \in B} \mathrm{C}(\beta) \Psi(\beta)=\Psi_{J} \otimes\left\{\bigotimes_{\alpha \in J^{c}} \phi_{\alpha}\right\}
$$

Q.E.D. 
It follows from this lemma that we can write

$$
\Psi=\lim _{J \uparrow} \Psi_{J} \otimes\left\{\otimes_{\alpha \in J^{\mathrm{c}}} \phi_{\infty}\right\}
$$

(see Eq. (3. 12)).

We now consider the associative law. ${ }^{24)}$ Let $K_{\beta}, \beta \in B$ be a partition of the index set $A$. We construct a unitary mapping between the ITPS

$$
H=\underset{A \in \infty}{\otimes(\otimes p)_{\infty}} H_{\infty}
$$

and the ITPS

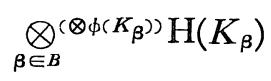

where

$$
\mathrm{H}\left(K_{\beta}\right)=\underset{\alpha \in K_{\beta}}{\otimes^{(\otimes \phi}(\omega)} H_{a}
$$

and

$$
\phi\left(K_{\beta}\right)=\otimes_{\alpha \in K_{\beta}} \phi_{a}
$$

For any product vector $\otimes \chi_{\infty} \in H$, let

$$
\chi\left(K_{\beta}\right)=\underset{\alpha \in K_{\beta}}{\otimes} \chi_{a}
$$

Then the mapping

$$
\bigotimes_{\alpha \in A} \chi_{\omega} \rightarrow \bigotimes_{\beta \in B} \chi\left(K_{\beta}\right)
$$

is one-to-one and isometric between total sets. Hence it can be extended to the desired unitary mapping. In practice we shall write

$$
H=\otimes_{\beta} \mathrm{H}\left(K_{\beta}\right)
$$

without explicitly writing the unitary operator. The preceding results are all given in the basic paper of von Neumann. ${ }^{2}$

We now show that any ITPS gives a complete atomic Boolean algebra of type I factors. For each $\alpha \in A$ let

$$
R_{a}=\mathrm{B}\left(H_{\omega}\right) \otimes\left(\underset{\beta \neq \infty}{\otimes} 1_{\beta}\right)
$$

Clearly

$$
R_{\alpha} \subset R_{\beta}^{\prime}, \quad \alpha \neq \beta
$$

24) J. von Neumann (reference 2), Sec. 4.2. 
It was shown by von Neumann that ${ }^{25)}$

$$
\bigvee_{a} R_{a}=\mathrm{B}(H)
$$

Thus $\left\{R_{\alpha}\right\}_{\alpha \in A}$ is a type I factorization (see definition 1.1). For any $J \subset A$, the associative law gives

$$
H=\left(\bigotimes_{\alpha \in J} H_{\omega}\right) \otimes\left(\bigotimes_{\alpha \in I c} H_{\omega}\right)
$$

Thus $^{25)}$

$$
\mathrm{R}(J)=\bigvee_{\alpha \in I} R_{\alpha}=\mathrm{B}(\mathrm{H}(J))
$$

is a type I factor, and we have a complete atomic Boolean algebra of type I factors.

We now generalize lemma 2. 6.

Lemma 3. 2: Let $H=\bigotimes_{a \in A}^{\left(\otimes \phi_{\infty}\right)} H_{a}$ be an ITPS, and let $\left\{R_{a}\right\}_{a \in A}$ be the associated type I factorization. Then $\Psi \in H$ is factorizable if and only if it is a product vector.

Proof: Clearly any product vector is factorizable. It remains only to prove the converse. By lemma 2.6 there exist minimal projections $P_{a} \in R_{\alpha}$ for all $\alpha \in A$ such that $P_{a} \Psi=\Psi$. We can assume $\|\Psi\|=1$. For any finite $J \subset A$, let $P(J)=\prod_{\alpha \in I} P_{a}$. Then $P(J) \Psi=\Psi$, and for any $Q \in R(J)$ we have

$$
P(J) Q \Psi=(\Psi, Q \Psi) \Psi
$$

Since the set of all such $Q \Psi$ is dense, it follows that

$$
P=\lim _{J \uparrow} P(J)
$$

exists and is the one-dimensional projection operator onto. $\Psi$. Now choose $\Psi_{a} \in H_{a}$ such that $P_{a} \Psi_{a}=\psi_{a},\left\|\Psi_{a}\right\|=1,\left(\Psi_{a}, \phi_{a}\right) \geqslant 0$. Since

$$
\left\|P \otimes \phi_{\omega}\right\|=\Pi\left(\Psi_{\omega}, \phi_{\omega}\right)
$$

it follows that, $\otimes \Psi_{a} \approx \otimes \phi_{a}$, hence $\otimes \Psi_{a} \in H$. Since $P \otimes \Psi_{a}=\otimes \Psi_{a}$, we have $\Psi=C \otimes \Psi_{a},|C|=1$. Q.E.D.

25) J. von Neumann (reference 2), Theorem IX. 
Lemma 3. 3: Let $H=\bigotimes_{a \in A}^{\left(\otimes \psi_{\infty}\right)} H_{a}$ be an ITPS. If

$$
\Phi=\underset{n \rightarrow \infty}{\text { weak }} \lim \Phi^{(n)}
$$

where each $\Phi^{(n)}$ is a product vector, then $\Phi$ is a product vector.

Proof: $\Phi^{(n)}$ is a product vector in a finite tensor product

$$
H=\left(\bigotimes_{i=1}^{n} H_{a_{i}}\right) \otimes H^{\prime}
$$

where $\left\{\alpha_{i}\right\}$ is any finite subset of $A$.

The proof of Lemma 2. 1 shows that the limit $\Phi$ is also a product vector, in this finite tensor product. Hence $\Phi$ is factorizable in $H=\otimes H_{a b}$. By Lemma 3.2 , it is a product vector.

\section{Complete Atomic Boolean Algebras of Type I Factors}

In this section we consider type I factorizations $\left\{R_{\omega}\right\}_{a \in A}$ (see definition 1. 1). If $J$ is a finite subset of $A$, then $\mathrm{R}(J)=\bigvee_{\alpha \in J} R_{a}$ is also a type I factor. ${ }^{26)}$ Thus we have an atomic Boolean algebra of type I factors. But if the factorization is obtained from an ITPS

$$
H=\bigotimes_{a \in A} H_{a}
$$

by

$$
R_{a}=\mathrm{B}\left(H_{a}\right) \otimes\left(\underset{\alpha^{\prime} \neq \alpha}{\otimes} \mathbb{1}_{a^{\prime}}\right)
$$

(where $\mathbb{1}_{\alpha}$ denotes the minimal von Neumann algebra of all multiples of the identity operator on $H_{a}$ ) then $\mathrm{R}(J)$ is type $\mathrm{I}$ for any $J \subset A$, and we have a complete atomic Boolean algebra of type I factors (see Eqs. (3.26-30)). We shall call any type I factorization $R_{a}$ a tensor product factorization (hereafter referred to as TPF) if it is unitarily equivalent to $R_{a b}$ obtained by Eq. (4.2). The main result of this section is that if $\Re$ is any complete atomic Boolean algebra of type I factors with atoms $\left\{R_{a}\right\}_{a \in A}$, then $R_{a}$ is a TPF (Theorem 4. 1).

26) F.J. Murray and J. von Neumann (reference 1), lemma 5.4.1. 
We begin with a discussion of what we call a partial tensor product factorization (hereafter referred to as a PTPF). Then we give an example of a countable type I factorization which gives a complete atomic Boolean algebra $\{\mathrm{R}(J)\}$ where $\mathrm{R}(J)$ is type $\mathrm{I}$ if either $J$ or $J^{\mathrm{c}}$ is finite, and is type II otherwise. Lemmas 4. 1-2 are useful technical lemmas. Lemmas 4. 3-4 give some rather obvious conditions that a type I factorization is a TPF. Lemma 4. 5 gives a necessary and sufficient condition that a PTPF is a TPF. Lemmas 4. 6-7 give further conditions that a type I factorization is a TPF. The key to proving our main result is the condition given by Eq. (4. 64). Lemma 4.8 proves that a type I factorization is a TPF if this condition holds. However the real motivation for this condition is in the proof of lemma 4.13, where we construct an $\mathrm{R}(J)$ which is not type I under the assumption that this condition does not hold. Lemma 4. 9 gives a condition that a countable type I factorization is a TPF. Lemmas 4. 1011 give sufficient conditions that a countable type I factorization is a PTPF. Lemma 4.12 is a cluster property used in the proof of lemma 4.13. Lemmas 4.8 and 4.13 prove our main result, which is stated as Theorem 4.1. Lemma 4.9 is the only lemma which is unnecessary for the proof of Theorem 4.1 as given here.

We begin with a discussion of what we call partial tensor products. Let $H_{a}, \alpha \in A$ be given, and consider a partition of the index set $A$ into finite subsets $K_{\beta}, \beta \in B$. Let

If $\alpha \in K_{\beta}$, let

$$
\mathrm{H}\left(K_{\beta}\right)=\bigotimes_{\alpha \in K_{\beta}} H_{\alpha}
$$

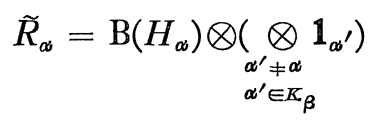

Choose some reference vector $\Psi_{\beta}$ in each $\mathrm{H}\left(K_{\beta}\right)$ and construct the ITPS

$$
H=\bigotimes_{\beta \in B} \mathrm{H}\left(K_{\beta}\right)
$$

If $\alpha \in K_{\beta}$, let

$$
R_{a b}=\widetilde{R}_{a} \otimes\left(\underset{\beta^{\prime} \neq \boldsymbol{\beta}}{\otimes} \mathbb{1}_{\beta^{\prime}}\right)
$$

Clearly $\left\{R_{\alpha}\right\}_{\alpha \in A}$ is a type I factorization. We shall call any type I factoriza- 
tion $R_{\omega}$ a partial tensor product factorization (hereafter referred to as PTPF) if it is unitarily equivalent to $R_{a}$ obtained by Eq. (4.6).

We consider the following type I factorization, which is based on an example given by von Neumann. ${ }^{27)}$ Let $H_{n i}, i \in 1,2, n \in I_{\infty}$ be twodimensional Hilbert spaces. Let $R_{n i}=\mathrm{B}\left(H_{n i}\right)$, and choose a unit reference vector $\Psi_{n}$ in each $H_{n 1} \otimes H_{n 2}$ such that

$$
\mathrm{d}\left(\Psi_{n} ; R_{n 1}, R_{n 2}\right)=1 / 2
$$

Let

$$
H=\bigotimes_{n=1}^{\infty}\left(H_{n 1} \otimes H_{n 2}\right)
$$

be the ITPS containing the unit product vector. $\Psi=\otimes \Psi_{n}$. Let

$$
\begin{aligned}
& R_{0}=\left(R_{11} \otimes \mathbb{1}_{11}\right) \otimes\left[\otimes_{n>1}\left(\mathbb{1}_{n 1} \otimes \mathbb{1}_{n 2}\right)\right] \\
& R_{n}=\left(\mathbb{1}_{n 1} \otimes R_{n 2}\right) \otimes\left(R_{n+1,1} \otimes \mathbb{1}_{n+1,2}\right) \otimes\left[\otimes_{m \neq n, n+1}\left(\mathbb{1}_{m 1} \otimes \mathbb{1}_{m 2}\right)\right]
\end{aligned}
$$

If either $J$ or $J^{\mathrm{c}}$ is finite then $\mathrm{R}(J)$ is type I. If both $J$ and $J^{\mathrm{c}}$ are infinite, then both $J, J^{\mathrm{c}}$ contain infinitely many $n$ such that either $n+1$ $\in J^{\mathrm{c}}, J$ or $n-1 \in J^{\mathrm{c}}, J$ respectively. It follows from von Neumann's results that $\mathrm{R}(J)$ is type II for this case. Thus we have a complete atomic Boolean algebra $\{\mathrm{R}(J)\}$ of type II and type I factors. Since the only type I factors are for finite or cofinite $J$, it does not contain any infinite complete Boolean subalgebra of type $I$ factors, which implies that $R_{n}$ is not a PTPF. ${ }^{28)}$

Lemma 4. 1: Given a Hilbert space $H$, a vector $\Psi \in H$, a projection $P$ and $a$ subset $S \subset H$. Then

(i) $(\Psi, \Phi) \geq \delta$ for all $\phi \in S$ implies $(\Psi, \Phi) \geq \delta$ for all $\Phi \in S^{(\mathrm{w})}$.

(ii) $(\Psi, \Phi)=0$ for all $\Phi \in S$ implies $(\Psi, \Phi)=0$ for all $\Phi \in S^{(w)}$.

(iii) $P \Phi=0$ for all $\Phi \in S$ implies $P \Phi=0$ for all $\Phi \in S^{(\mathrm{w})}$.

(iv) $P \Phi=\Phi$ for all $\Phi \in S$ implies $P \Phi=\Phi$ for all $\Phi \in S^{(\mathrm{w})}$.

27) J. von Neumann (reference 2), Sec. 7.3.

28) J.R. Klauder (private communication) has shown that by a suitable choice of basis for the space of test functions, the representation of the CCRs for a relativistic free scalar field gives a type I factorization which is not a PTPF. 
Proof: We prove (i). Since

$$
\Phi \in S-\delta \Psi /\|\Psi\|^{2}
$$

satisfies

$$
(\Psi, \Phi)>0
$$

we may take $\delta=0$. Let $\Phi \in S^{(\mathrm{w})}$ and assume

$$
(\Psi, \Phi)=\varepsilon<0
$$

Then the weak neighborhood

$$
N(\Phi ; \Psi, \varepsilon / 2)=\{\chi:|(\Psi, \Phi-\chi)|<\varepsilon / 2\}
$$

is disjoint from $S$, which contradicts $\Phi \in S^{(\mathrm{w})}$.

Clearly (i) implies (ii), and (iii) is equivalent to (iv) (let $P \rightarrow 1-P$ ). (iv) is equivalent to $P \chi=0$ implies $(\chi, \Phi)=0$ which follows from (ii). Q.E.D.

Lemma 4. 2: Let $\quad H=H_{1} \otimes H_{2}, R_{1}=\mathrm{B}_{1}\left(H_{1}\right) \otimes 1_{2}, R_{2}=1_{1} \otimes \mathrm{B}\left(H_{2}\right)$. Let $P^{(n)} \in R_{1}, n \in I_{\infty}$ be minimal projections with

$$
\underset{n \rightarrow \infty}{\text { weak }} \lim ^{(n)}=\lambda P
$$

and let $Q^{(n)} \in R_{2}$ be such that $\left\|Q^{(n)}\right\| \leqq 1$ and

$$
\underset{n \rightarrow \infty}{\text { weak }} \lim P^{(n)} Q^{(n)} \Psi=\Phi
$$

Then

$$
\Phi=\underset{n \rightarrow \infty}{\operatorname{weak}} \lim \lambda P Q^{(n)} \Psi
$$

Proof: Let $\Phi_{1}^{(n)}, \Phi_{1}$ be unit vectors in $H_{1}$ such that

$$
\begin{aligned}
& P^{(n)} \Phi_{1}^{(n)}=\Phi_{1}^{(n)} \\
& P \Phi_{1}=\Phi_{1} \\
& \left(\Phi_{1}^{(n)}, \Phi_{1}\right) \geq 0
\end{aligned}
$$

We prove that

$$
\sqrt{\lambda} \Phi_{1}=\underset{n \rightarrow \infty}{\operatorname{weak}} \lim \Phi_{1}^{(n)}
$$

From Eq. (4.15) we have 


$$
\lim _{n \rightarrow \infty}\left|\left(\Phi_{1}, \Phi_{1}^{(n)}\right)\right|^{2}=\lim _{n \rightarrow \infty}\left(\Phi_{1}, P^{(n)} \Phi_{1}\right)=\lambda
$$

hence

$$
\lim _{n \rightarrow \infty}\left(\Phi_{1}, \Phi_{1}^{(n)}\right)=\sqrt{\lambda}
$$

For $\left(\chi, \Phi_{1}\right)=0$ we have

$$
\lim _{n \rightarrow \infty}\left|\left(\chi, \Phi_{1}^{(n)}\right)\right|^{2}=\lim _{n \rightarrow \infty}\left(\chi, P^{(n)} \chi\right)=0
$$

which proves Eq. (4.21). Let

$$
\Psi=\sum \lambda_{i} \Psi_{1 i} \otimes \Psi_{2 i}
$$

be the standard diagonal espansion of $\Psi$ given by Eq. (2.9). Then

$$
\begin{aligned}
& P^{(n)} Q^{(n)} \Psi=\Phi_{1}^{(n)} \otimes Q^{(n)} \Phi_{2}^{(n)} \\
& \lambda P Q^{(n)} \Psi=\sqrt{ } \lambda \Phi_{1} \otimes Q^{(n)} \Phi_{n}^{(\infty)}
\end{aligned}
$$

where

$$
\begin{aligned}
& \Phi_{2}^{(n)}=\sum \lambda_{i}\left(\Phi_{1}^{(n)}, \Psi_{1 i}\right) \Psi_{2 i} \\
& \Phi_{n}^{(\infty)}=\sum \lambda_{i} \sqrt{\lambda}\left(\Phi_{1}, \Psi_{1 i}\right) \Psi_{2 i}
\end{aligned}
$$

From the equivalence of weak and $\sigma$ weak topology for a norm bounded set, it follows that

$$
\left\|\Phi_{2}^{(n)}-\Phi_{n}^{(\infty)}\right\|^{2}=\sum \lambda_{i}^{2}\left(\Psi_{1 i}, P^{(n)^{\prime}} \Psi_{1 i}\right) \rightarrow 0
$$

where $P^{(n)^{\prime}}$ is the projection on the space spanned by the vector $\Phi_{1}^{(n)}$ $-\sqrt{ } \lambda \Phi_{1}$ times the norm squared of this vector and tends to zero weakly. Eq. (4. 21), (4.30) and $\left\|Q^{(n)}\right\| \leqq 1$ implies (4. 17).

Lemma 4. 3: Let $\left\{R_{a}\right\}_{a \in A}$ be a type I factorization. If there exists a factorizable vector $\Phi$ (see Eq. (2.16) then $R_{a j}$ is a TPF.

Proof: We can assume $\|\Phi\|=1$. For each $\alpha \in A$ we have

$$
\begin{aligned}
& H=H_{a} \otimes H_{\alpha}^{\prime}, \\
& R_{a}=\mathrm{B}\left(H_{\omega}\right) \otimes 1
\end{aligned}
$$

By lemma 2. 6 there exists a minimal $P_{a} \in R_{a}$ such that

$$
\Phi=P_{a} \Phi
$$

It follows that 


$$
\Phi=\Phi_{a j} \otimes \Phi_{\alpha}^{\prime}
$$

where we can take $\left\|\Phi_{a i}\right\|=1$. Now consider the ITPS

$$
\bigotimes_{\alpha \in A}^{\left(\otimes \Phi^{\prime}\right)} H_{a}
$$

By a slight abouse of notation, we shall let $Q_{a}$ denote the corresponding operators in $R_{a}, \mathrm{~B}\left(H_{a}\right)$ andB $\left(\otimes H_{a}\right)$. For any $Q_{a} \in R_{a}$ and all $n$, consider the map

$$
\prod_{i=1}^{n} Q_{\omega_{i}} \Phi \rightarrow \prod_{i=1}^{n} Q_{\omega_{i}}\left(\otimes \Phi_{\omega_{i}}\right)
$$

This mapping is one-to-one and isometric between total sets. Therefore it can be extended by linearity and continuity to a unitary operator $U$ from $H$ onto $\otimes H_{a}$. By construction

Q.E.D.

$$
U R_{a} U^{-1}=\mathrm{B}\left(H_{a}\right) \otimes\left(\underset{\alpha^{\prime} \neq \neq^{\alpha}}{\otimes} \mathbb{1}_{a^{\prime}}\right)
$$

Lemma 4. 4: Let $\left\{R_{a i}\right\}_{a \in A}$ be a type I factorization. If there exists a vector $\Psi$, and a minimal projection $P_{a}$ in each $R_{a}$ such that

$$
\left(\Psi, \prod_{a \in J} P_{a} \Psi\right) \geq \varepsilon>0
$$

for all finite $J \subset A$, then $R_{a}$ is a TPF.

Proof: For any finite $J$ let $\mathrm{P}(J)=\prod_{\alpha \in J} P_{a}, \Psi(J)=\mathrm{P}(J) \Psi$, and

$$
\mathrm{S}(J)=\left\{\bigcup_{K \supset J} \Psi(K)\right\}
$$

where $K$ is finite. Since the unit sphere in Hilbert space is weakly compact, the set $S=\bigcap_{J} \mathrm{~S}(J)^{(\mathrm{w})}$ is nonempty. Let $\Phi \in S$. By Eq. (4. 37) and lemma 4. 1 we have

$$
(\Psi, \Phi) \geq \varepsilon
$$

thus $\Phi \neq 0$. If $\alpha \in J$, then $P_{a} \Psi(J)=\Psi(J)$ and lemma 4.1 gives

$$
P_{\alpha} \Phi=\Phi
$$

It follows from lemma 2.6 that $\Phi$ is factorizable. Thus $R_{a}$ is a TPF by lemma 4. 3. Q.E.D. 
Lemma 4. 5: Let $\left\{R_{\alpha}\right\}_{\alpha \in A}$ be a PTPF. If all $R(J)$ are type $I$, then $R_{\infty}$ is a $T P F$.

Proof: We have

$$
H=\bigotimes_{\beta \in B}^{\left(\otimes \Phi_{\beta}\right)} \mathrm{H}\left(K_{\beta}\right)
$$

For any $J \subset A$ let

$$
\begin{aligned}
& J_{\beta}=J \cap K_{\beta} \\
& J_{\beta}^{\prime}=J^{\mathrm{c}} \cap K_{\beta}
\end{aligned}
$$

where either $J_{\beta}$ or $J_{\beta}^{\prime}$ may be empty. We have

$$
\mathrm{H}\left(K_{\beta}\right)=\mathrm{H}\left(J_{\beta}\right) \otimes \mathrm{H}\left(J_{\beta}^{\prime}\right)
$$

where the factor space is one-dimensional if the set is empty. Thus

$$
H=\underset{\beta \in B}{\otimes}\left(\otimes \Phi_{\beta}\right)\left\{\mathrm{H}\left(J_{\beta}\right) \otimes \mathrm{H}\left(J_{\beta}^{\prime}\right)\right\}
$$

and the factor

$$
\left.\mathrm{R}(J)=\bigotimes_{\beta \in B}\left\{\mathrm{~B}\left(\mathrm{H}\left(J_{\beta}\right)\right) \otimes \mathbb{1}\right)\right\}
$$

is now in the standard form considered by Araki, ${ }^{29)}$ who proved that $\mathrm{R}(J)$ is type $I$ if and only if

$$
\sum_{\beta \in B}\left\{1-\mathrm{d}\left(\Phi_{\beta} ; \mathrm{R}\left(J_{\beta}\right), \mathrm{R}\left(J_{\beta}^{\prime}\right)\right)\right\}<\infty
$$

Thus all $\mathrm{R}(J)$ type $\mathrm{I}$ implies that

$$
\sum_{\beta \in B}\left\{1-\inf _{J_{\beta}} \mathrm{d}\left(\Phi_{\beta} ; \mathrm{R}\left(J_{\beta}\right), \mathrm{R}\left(J_{\beta}^{\prime}\right)\right)\right\}<\infty
$$

It follows from lemma 2.7 that

$$
\sum_{\beta}\left\{1-\mathrm{d}\left(\Phi_{\beta} ; R_{\alpha}, \alpha \in K_{\beta}\right)\right\}<\infty
$$

which implies that ${ }^{23)}$

$$
\Pi_{\beta} \mathrm{d}\left(\Phi_{\beta} ; R_{a}, \alpha \in K_{\beta}\right) \neq 0
$$

Hence there exist minimal projections $P_{a} \in R_{a}$ such that $\Phi=\otimes \Phi_{\beta}$ satisfies the conditions of lemma 4. 4. Q.E.D.

29) H. Araki, J. Math. Phys. 4, 1343 (1963), Theorem 5 (especially Eq. (10.38)). 
Lemma 4.6: Let $\left\{R_{i}\right\}_{i \in I_{\infty}}$ be a countable type I factorization. If there exists a vector $\Psi$, and minimal projections $P_{j}^{(n)} \in R_{j}, j \in I_{n}$ for each $n$ such that

$$
\lim _{n \rightarrow \infty}\left(\Psi, \prod_{j=1}^{n} P_{j}^{(n)} \Psi\right)>0
$$

then $R_{i}$ is a TPF.

Proo: Let $\mathrm{P}(n)=\prod_{j=1}^{n} P_{j}^{(n)}$. Then by weak sequential compactness there exists a subsequence $n_{k}$ such that

$$
\underset{k \rightarrow \infty}{\operatorname{weak}} \lim \mathrm{P}\left(n_{k}\right) \Psi=\Phi
$$

Then

$$
(\Psi, \Phi)=\lim _{k \rightarrow \infty}\left(\Psi, \mathrm{P}\left(n_{k}\right) \Psi\right)>0
$$

Now for each $j$ and $n>j$ we have

$$
\begin{aligned}
(\Psi, \mathrm{P}(n) \Psi) & =\left(P_{j}^{(n)} \Psi, \mathrm{P}(n) P_{j}^{(n)} \Psi\right) \\
& \leq\left\|P_{j}^{(n)} \Psi\right\|^{2}=\left(\Psi, P_{j}^{(n)} \Psi\right)
\end{aligned}
$$

Thus

$$
\lim _{n \rightarrow \infty}\left(\Psi, P_{j}^{(n)} \Psi\right)>0
$$

Since $P_{j}^{(m)}$ is a minimal projection, we may use the weak sequential compactness in $H_{j}$. Thus there is a subsequence of $n_{k}$ such that $P_{1}^{(n)}$ converges weakly. Choose a subsequence of this subsequence such that $P_{2}^{(n)}$ converges weakly, and repeat this process for each $j$. Let $N_{k}$ be the diagonal subsequence, then by $(4.52)$

$$
\underset{k \rightarrow \infty}{\text { weak }} \lim \mathrm{P}\left(N_{k}\right) \Psi=\Phi
$$

and by construction

$$
\underset{k \rightarrow \infty}{\text { weak }} \lim P_{j}^{\left(N_{k}\right)}=\lambda_{j} P_{j}
$$

where $P_{j}$ is a minimal projection in $R_{j}$, and $0<\lambda_{j} \leq 1$. It follows from lemma 4.2 that

$$
\Phi=\underset{k \rightarrow \infty}{\operatorname{weak}} \lim \lambda_{j} P_{j}\left\{\prod_{i \neq j} P_{i}^{\left(N_{k}\right)}\right\} \Psi
$$


which implies that

$$
\Phi=P_{j} \Phi
$$

for any $j$. It now follows from lemma 4.4 that $R_{i}$ is a TPF. Q.E.D.

Lemma 4. 7: Let $\left\{R_{\alpha}\right\}_{\infty \ni A}$ be a type I factorization such that all $R(J)$ are type $I$. If there exists a vector $\Psi$ such that

$$
\inf \mathrm{d}\left(\Psi ; R_{\omega_{1}}, \cdots, R_{\omega_{n}}, \mathrm{R}\left(\left\{\alpha_{1}, \cdots, \alpha_{n}\right\}^{\mathrm{c}}\right)\right)=\varepsilon>0
$$

where inf is taken over all $n, \alpha_{1} \cdots, \alpha_{n}$, then $R_{\alpha}$ is a TPF.

Proof: We first prove that the number of $\alpha \in A$ such that

$$
\mathrm{d}\left(\Psi ; R_{a}, R_{a}^{\prime}\right)<1-\delta, \delta>0
$$

is finite. Suppose there exists a countably infinite set of $\alpha_{i}, i \in I_{\text {o }}$ satisfying Eq. (4.61). Consider the countable type $I$ factorization $\left\{\mathrm{R}\left(\left\{\alpha_{i}, i \in I_{\infty}\right\}^{\mathrm{c}}\right), \mathrm{R}_{\alpha_{i}}, i \in I_{\infty}\right\}$. It follows from Eq. (4.60) and lemma 4.6 that this factorization is a TPF. Using lemmas 2.4 and 3.1 , this implies that

$$
\lim _{i \rightarrow \infty} \mathrm{d}\left(\Psi ; R_{\alpha_{i} i}, R_{\alpha_{i} i}^{\prime}\right)=1
$$

which contradicts Eq. (4.61). It follows by a standard argument that the subset

$$
A_{0}=\left\{\alpha \in A: \mathrm{d}\left(\Psi ; R_{a}, R_{\alpha}^{\prime}\right) \neq 1\right\}
$$

is countable. If $\alpha \in A_{0}^{c}$, there is a minimal projection $P_{\alpha} \in R_{\alpha}$ such that $P_{a} \Psi=\Psi$, hence $\left\{R_{a}\right\}_{a \in A_{0}^{\mathrm{c}}}$ is a TPF by lemma 4. 4. It follows from Eq. (4.60) and lemma 4. 6 that $\left\{R_{a}\right\}_{a \in A_{0}}$ is a TPF. Hence $\left\{R_{a}\right\}_{a \in A}$ is a TPF. Q.E.D.

Lemma 4. 8: Let $\left\{R_{a i}\right\}_{a \in A}$ be a type I factorization such that all $R(J)$ are type $I$. If there exists some unit vector $\Psi$ such that

$$
\sup _{K} \inf _{J \subset K^{\mathrm{c}}} \mathrm{d}\left(\Psi ; \mathrm{R}(J), \mathrm{R}\left(J^{\mathrm{c}}\right)\right)=1
$$

where $J, K$ are finite subsets, then $R_{a}$ is a TPF.

Proof: Choose $\varepsilon^{\prime}$ such that $\varepsilon^{\prime}<\varepsilon$ and $\delta \cdot \varepsilon^{\prime}<1$ where $\varepsilon, \delta$ are given by lemma 2.7. Choose a finite $K^{\prime}$ such that 


$$
1-\inf _{J \subset K^{\mathrm{c}}} \mathrm{d}\left(\Psi ; \mathrm{R}(J), R\left(J^{\mathrm{c}}\right)\right) \leq \varepsilon^{\prime}
$$

For any finite $J=\left\{\alpha_{1}, \cdots, \alpha_{n}\right\} \subset K^{\mathrm{c}}$ it follows from lemma 2.7 that

$$
1-\mathrm{d}\left(\Psi ; R_{\omega_{1}}, \cdots, R_{\omega_{n}}, \mathrm{R}\left(J^{\mathrm{c}}\right)\right) \leq \delta \cdot \varepsilon^{\prime}<1
$$

By lemma 4.7 this implies that $R_{a}$ is a TPF. Q.E.D.

Lemma 4.9: Let $\left\{R_{i}\right\}_{i \in I_{\infty}}$ be a countable type I factorization. If there exists a vector $\Psi$ such that

$$
\sum_{n=1}^{\infty}\left\{1-\mathrm{d}\left(\Psi ; \mathrm{R}\left(I_{n}\right), \mathrm{R}\left(I_{n}^{\mathrm{c}}\right)\right)\right\}<\infty
$$

then $R_{i}$ is a TPF.

Proof: Let

$$
\varepsilon_{n}=1-\mathrm{d}\left(\Psi ; \mathrm{R}\left(I_{n}\right), \mathrm{R}\left(I_{n}^{\mathrm{c}}\right)\right)
$$

By lemma 2. 3 there exist minimal projections $P_{n} \in \mathrm{R}\left(I_{n}\right), P_{n}^{\prime} \in \mathrm{R}\left(I_{n}^{\mathrm{c}}\right)$ such that

$$
\left(\Psi, P_{n} \Psi\right)=\left(\Psi, P_{n}^{\prime} \Psi\right)=1-\varepsilon_{n}
$$

which is equivalent to

$$
\left\|\left(1-P_{n}\right) \Psi\right\|^{2}=\|\left.\left(1-P_{n}^{\prime}\right) \Psi !\right|^{2}=\varepsilon_{n}
$$

Consider the minimal projection

$$
P_{n}^{\prime \prime}=P_{n-1} P_{n}^{\prime}
$$

in $R_{n}^{\prime}$. We have

$$
P_{n}^{\prime \prime} \Psi-\Psi=P_{n-1}\left(P_{n}^{\prime} \Psi-\Psi\right)-\left(1-P_{n-1}\right) \Psi
$$

hence

$$
\begin{aligned}
\left\|P_{n}^{\prime \prime} \Psi-\Psi\right\|^{2} & =\left\|P_{n-1}\left(P_{n}^{\prime} \Psi-\Psi\right)\right\|^{2}+\left\|P_{n-1} \Psi-\Psi\right\|^{2} \\
& \leq\left\|P_{n}^{\prime} \Psi-\Psi\right\|^{2}+\left\|P_{n-1} \Psi-\Psi\right\|^{2} \\
& =\varepsilon_{n}+\varepsilon_{n-1}
\end{aligned}
$$

which is equivalent to

$$
1-\left(\Psi, P_{n}^{\prime \prime} \Psi\right) \leq \varepsilon_{n}+\varepsilon_{n-1}
$$

It follows from lemma 2. 3 that there exists a minimal projection $Q_{n} \in R_{n}$ 
such that

$$
\left\|Q_{n} \Psi-\Psi\right\|^{2} \leq \varepsilon_{n}+\varepsilon_{n-1}
$$

Choose $n$ such that

$$
\sum_{j=n}^{\infty}\left(\varepsilon_{j}+\varepsilon_{j-1}\right)<1 / 2
$$

Let

$$
\Psi_{m}^{n}=\left(\prod_{j=n}^{n+m} Q_{j}\right) \Psi
$$

then

$$
\begin{aligned}
& \left\|\Psi_{m}^{n}-\Psi_{m+k}^{n n}\right\|^{2}=\left\|\sum_{j=1}^{k}\left(\prod_{l=n}^{n ! m+j-1} Q_{l}\right)\left(Q_{n+m+j} \Psi-\Psi\right)\right\|^{2} \\
& \leq \sum_{j=1}^{k}\left(\varepsilon_{n+m+j}+\varepsilon_{n+m+j-1}\right)
\end{aligned}
$$

Thus $\Psi_{m}^{n}$ converges strongly to a vector $\Psi^{n}$ and $\left\|\Psi^{n}-\Psi\right\|^{2}<\sum_{j=0}^{\infty}\left(\varepsilon_{n+j}\right.$ $\left.+\varepsilon_{n+j-1}\right)$. Hence $\Psi \neq 0$ implies $\Psi^{n} \neq 0$ for large $n$. For $j \geq n$ we have

$$
Q_{j} \Psi^{n}=\Psi^{n}
$$

Since one can always find minimal projections $Q_{j}^{\prime} \in R_{j}, j \in I_{n-1}$ such that

$$
\left(\Psi^{n}, \prod_{j=1}^{n-1} Q_{j}^{\prime} \Psi^{n}\right)>0
$$

the conditions of lemma 4.4 are satisfied and $R_{n}$ is a TPF. Q.E.D.

Lemma 4.10: Let $\left\{R_{n}\right\}_{n \in I_{\infty}}$ be a countable type I factorization. Let $J_{n}$ be any increasing sequence of finite subsets such that $\cup_{n} J_{n}=I_{\infty}$. Then the following conditions are equivalent

(i) there exists some unit vector $\Psi$ such that

$$
\lim _{n \rightarrow \infty} \mathrm{d}\left(\Psi ; \mathrm{R}\left(J_{n}\right), \mathrm{R}\left(J_{n}^{\mathrm{c}}\right)\right)=1
$$

(ii) there exist minimal projections $P_{n} \in R\left(J_{n}^{\mathrm{c}}\right)$ such that $P_{n} \rightarrow 1$ strongly.

(iii) all unit vectors $\Psi$ satisfy $E q$. (4. 80). Any of these conditions implies that $R_{n}$ is a PTPF.

Proof: We first show that (ii) implies (iii). For any unit vector $\Psi$ we have 


$$
\lim _{n \rightarrow \infty} \mathrm{d}\left(\Psi ; \mathrm{R}\left(J_{n}\right), \mathrm{R}\left(J_{n}^{\mathrm{c}}\right)\right) \geq \lim _{n \rightarrow \infty}\left(\Psi, P_{n} \Psi\right)=1
$$

Clearly (iii) implies (i). It remains to prove that (i) implies (ii). For each $n$ we have

$$
H=\mathrm{H}\left(J_{n}\right) \otimes \mathrm{H}\left(J_{n}^{\mathrm{c}}\right)
$$

Let

$$
\Psi=\sum_{i=1}^{\infty} \lambda_{i}^{(n)} \alpha_{i}^{(n)} \otimes \beta_{i}^{(n)}
$$

be the standard diagonal expansionf of $\Psi$ given by Eq. (2.12). It follows from lemma 2. 2 and Eq. (4. 80) that $\lambda_{1}^{(n)} \rightarrow 1$ as $n \rightarrow \infty$. Let $P_{n}$ be the minimal projection associated with the vector $\beta_{1}^{(n)} \in \mathrm{H}\left(I_{n}^{\mathrm{c}}\right)$, then $P_{n} \Psi \rightarrow \Psi$ strongly. We now prove that $P_{n} \Phi \rightarrow \Phi$ strongly for all $\Phi \in H$.

First, let $U$ be a unitary operator in $\mathrm{R}\left(J_{n}\right)$. Then, for $\Phi=U \Psi$,

$$
\left\|P_{m} \Phi-\Phi\right\|^{2}=\mid i P_{m} \Psi-\Psi \|^{2} \rightarrow 0
$$

Since the union of $\mathrm{R}\left(J_{n}\right)$ is irreducible, the vectors of the form $\Phi=U \Psi$ are dense in the Hilbert space. Since $\left\|P_{n}\right\|=1,\left(P_{n}-1\right) \Phi \rightarrow 0$ for a dense set of $\Phi$ implies the same for all $\Phi$.

Finally we show that (i) implies that $R_{n}$ is a PTPF. We can choose a subsequence $n_{k}$ such that

$$
\sum_{k=1}^{\infty}\left\{1-\mathrm{d}\left(\Psi ; \mathrm{R}\left(J_{n_{k}}\right), \mathrm{R}\left(J_{n_{k}}^{\mathrm{c}}\right)\right)\right\}<\infty
$$

The result then follows from lemma 4.9. Q.E.D.

Lemma 4.11: Let $\left\{R_{i}\right\}_{i \in I_{\infty}}$ be a countable type I factorization. Let d be a density matrix (nonnegative trace class operator with $\operatorname{Tr} d=1$ ). Let $J_{n}$ be any increasing sequence of finite subsets such that $\cup_{n} J_{n}=I_{\infty}$. If for each $n$ there exist density matrices $d_{n} \in \mathrm{R}\left(J_{n}\right), d_{n}^{\prime} \in \mathrm{R}\left(J_{n}^{\mathrm{c}}\right)$ such that for all $Q_{1} \in \mathrm{R}\left(J_{n}\right), Q_{2} \in \mathrm{R}\left(J_{n}^{\mathrm{c}}\right)$

$$
\left|\operatorname{Tr}\left(d Q_{1} Q_{2}\right)-\operatorname{Tr}\left(d_{n} Q_{1}\right) \operatorname{Tr}\left(d_{n}^{\prime} Q_{2}\right)\right|<\varepsilon_{n}|| Q_{1}|| \| Q_{2}||
$$

where $\lim \varepsilon_{n}=0_{i}$ then $R$, is a PTPF.

Proof: Let $\Psi$ be an eigen vector of $d$ belonging to the eigenvalue $\lambda \neq 0$, $\|\Psi\|=1$, and let $P$ be the associated one-dimensional projection. Since 
the union of $\mathrm{R}\left(J_{n}\right)$ is irreducible, it follows from von Neumann's density theorem $^{30)}$ that its unit sphere is dense in the unit sphere of $\mathrm{B}(H)$ in the strongest operator topology. ${ }^{31}$ It follows that, given $\varepsilon>0$, there exists a sufficiently large integer $n$ and an operator $P_{n} \in \mathrm{R}\left(J_{n}\right),\left\|P_{n}\right\| \leq 1$ such that

$$
\left\|\left(P-P_{n}\right) d^{1 / 2}\right\|_{\mathrm{HS}}=\left\|d^{1 / 2}\left(P-P_{n}^{*}\right)\right\|_{\mathrm{HS}}<\varepsilon
$$

where $\|T\|_{\text {HS }}$ is the Hilbert-Schmidt norm defined by

$$
\|T\|_{\mathrm{HS}}=\left[\operatorname{Tr} T^{*} T\right]^{1 / 2} \geq\|T\|
$$

For any $Q \in \mathrm{B}(H)$ consider

$$
\operatorname{Tr}\left(P d P-P_{n} d P_{n}^{*}\right) Q=\lambda(\Psi, Q \Psi)-\operatorname{Tr}\left(P_{n} d P_{n}^{*} Q\right)
$$

We have

$$
P d P-P_{n} d P_{n}^{*}=\left(P-P_{n}\right) d P+P_{n} d\left(P-P_{n}^{*}\right)
$$

Since

$$
|\operatorname{Tr} S T| \leq\|S\|_{\mathrm{HS}}\|T\|_{\mathrm{HS}}
$$

we have

$$
\begin{gathered}
\left|\operatorname{Tr}\left(P d P-P_{n} d P_{n}^{*}\right) Q\right| \leq\left.\left\|\left(P-P_{n}\right) d^{1 / 2}\right\|\right|_{\mathrm{HS}}\left\|d^{1 / 2} P Q\right\|_{\mathrm{HS}} \\
+\left\|Q P_{n} d^{1 / 2}\right\|\left\|_{\mathrm{HS}}\right\| d^{1 / 2}\left(P-P_{n}^{*}\right) ! \|_{\mathrm{HS}}
\end{gathered}
$$

Now

$$
\left\|d^{1 / 2} P Q\right\|_{\mathrm{HS}}^{2}=\operatorname{Tr} Q^{*} P d P Q=\lambda\left(\Psi, Q Q^{*} \Psi\right)
$$

which gives

$$
\left\|d^{1 / 2} P Q\right\|_{\mathrm{HS}} \leq \lambda^{1 / 2}\|Q\|
$$

We have

$$
Q P_{n} d^{1 / 2}=Q P d^{1 / 2}+Q\left(P_{n}-P\right) d^{1 / 2}
$$

Since

$$
\|P\|_{\mathrm{HS}}=\|\left. d^{1 / 2}\right|_{\mathrm{HS}}=1
$$

it follows from Eqs. (4. 95), (4. 94) and (4. 87) that

30) See J. Dixmier (reference 15), Sec. I.3.

31) The strongest topology is defined by the semi-norms $T \rightarrow\left[\left.\sum|| T x_{i}\right|^{2}\right]^{1 / 2}$ where $\left\{x_{i}\right\}$ is any sequence of vectors such that $\sum|| x_{i}||^{2}<\infty$. An equivalent definition is to use the semi-norms $T \rightarrow\left[\operatorname{Tr} d T^{*} T\right]^{1 / 2}$ where $d$ is any nonnegative trace class operator. 


$$
\left\|Q P_{n} d^{1 / 2}\right\|_{\mathrm{HS}} \leq\|Q\|\left(\lambda^{1 / 2}+\varepsilon\right)
$$

Eqs. (4. 92), (4. 94), (4. 97) and (4. 87) now give

$$
\left|\operatorname{Tr}\left(P d P-P_{n} d P_{n}^{*}\right) Q\right| \leq \varepsilon\left(2 \lambda^{1 / 2}+\varepsilon\right)|| Q \|
$$

which implies

$$
\left|\lambda(\Psi, Q \Psi)-\operatorname{Tr}\left(P_{n} d P_{n}^{*} Q\right)\right| \leq \varepsilon\left(2 \lambda^{1 / 2}+\varepsilon\right)|| Q \|
$$

for any $Q \in \mathrm{B}(H)$. Now let $Q=Q_{1} Q_{2}$ where $Q_{1} \in \mathrm{R}\left(J_{n}\right), Q_{2} \in \mathrm{R}\left(J_{n}^{\mathrm{c}}\right)$. Then

$$
\operatorname{Tr}\left(P_{n} d P_{n}^{*} Q_{1} Q_{2}\right)=\operatorname{Tr}\left[d\left(P_{n}^{*} Q_{1} P_{n}\right) Q_{2}\right]
$$

and it follows from Eqs. (4.86) and (4.99) that

$$
\left|\left(\Psi, Q_{1} Q_{2} \Psi\right)-\operatorname{Tr}\left(\tilde{d}_{n} Q_{1}\right) \operatorname{Tr}\left(\tilde{d}_{n}^{\prime} Q_{2}\right)\right|<\varepsilon^{\prime}|| Q_{1}|!|\left|Q_{2}\right| \mid
$$

where

$$
\begin{aligned}
& \tilde{d}_{n}=\lambda^{-1} P_{n} d P_{n}^{*} \\
& \varepsilon^{\prime}=\left[\varepsilon_{n}+\varepsilon\left(2 \lambda^{1 / 2}+\varepsilon\right)\right] / \lambda
\end{aligned}
$$

By letting $Q_{2}=1$ we get

$$
\left|\left(\Psi, Q_{1} \Psi\right)-\operatorname{Tr}\left(\tilde{d}_{n} Q_{1}\right)\right|<\varepsilon^{\prime}|| Q_{1} \|
$$

By letting $Q_{1}=1$ we get

$$
\mid\left(\Psi, Q_{2} \Psi-\operatorname{Tr}\left(\tilde{d}_{n}\right) \operatorname{Tr}\left(d_{n}^{\prime} Q_{2}\right) \mid<\varepsilon^{\prime}\left\|Q_{2}\right\|\right.
$$

By letting $Q_{1}=Q_{2}=1$ we get $\left|1-\operatorname{Tr} \tilde{d}_{n}\right|<\varepsilon^{\prime}$, hence

$$
\left|\left(\Psi, Q_{2} \Psi\right)-\operatorname{Tr}\left(d_{n}^{\prime} Q_{2}\right)\right|<2 \varepsilon^{\prime}|| Q_{2}||
$$

Eqs. (4. 101), (4. 104), (4. 106) now give

$$
\left|\left(\Psi, Q_{1} Q_{2} \Psi\right)-\left(\Psi, Q_{1} \Psi\right\rangle\left(\Psi, Q_{2} \Psi\right)\right|<4 \varepsilon^{\prime}|| Q_{1} \||| Q_{2}||
$$

Since $\varepsilon$ was arbitrary, and $\lim \varepsilon_{n}=0$, it follows from lemma 2.5 that

$$
\lim _{n \rightarrow \infty} \mathrm{d}\left(\Psi ; \mathrm{R}\left(J_{n}\right), \mathrm{R}\left(J_{n}^{\mathrm{c}}\right)\right)=1
$$

and lemma 4. 10 implies that $R_{i}$ is a PTPF. Q.E.D.

Lemma 4.12: Let $\left\{R_{\alpha}\right\}_{\omega \in A}$ be a type I factorization. Given any unit vector $\Psi$, any finite subset $J \subset A$, and any $\varepsilon>0$, there exists a finite $K \supset J$ such that for all $Q_{1} \in \mathrm{R}(J), Q_{2} \in \mathrm{R}\left(K^{\mathrm{c}}\right)$ we have ${ }^{32)}$ 


$$
\left|\left(\Psi, Q_{1} Q_{2} \Psi\right)-\left(\Psi, Q_{1} \Psi\right)\left(\Psi, Q_{2} \Psi\right)\right|<\varepsilon|| Q_{1}\|\| Q_{2} \|
$$

Proof: Since $J$ is finite, $\mathrm{R}(J)$ is type $\mathrm{I}$ and

$$
H=\mathrm{H}(J) \otimes \mathrm{H}\left(J^{\mathrm{c}}\right)
$$

Let

$$
\Psi=\sum_{i=1}^{\infty} \lambda_{i} \alpha_{i} \otimes \beta_{i}
$$

be the standard diagonal expansion of $\Psi$ (see Eq. (2.12)). For any $Q_{1} \in \mathrm{R}(J), Q_{2} \in \mathrm{R}\left(J^{\mathrm{c}}\right)$ we have

$$
\begin{aligned}
& \left(\Psi, Q_{1} \Psi\right)=\sum \lambda_{i}^{2}\left(\alpha_{i}, Q_{1} \alpha_{i}\right) \\
& \left(\Psi, Q_{1} Q_{2} \Psi\right)=\sum \lambda_{i} \lambda_{j}\left(\alpha_{i}, Q_{1} \alpha_{j}\right)\left(\beta_{i}, Q_{2} \beta_{j}\right)
\end{aligned}
$$

For any $\varepsilon^{\prime}>0$ we can choose $n$ such that

$$
\sum_{i=n+1}^{\infty} \lambda_{i}^{2}<\varepsilon^{\prime}
$$

Then

$$
\begin{aligned}
\mid\left(\Psi, Q_{1} Q_{2} \Psi\right) & -\left(\Psi, Q_{1} \Psi\right)\left(\Psi, Q_{2} \Psi\right) \mid \\
& <\left|\sum_{i, j=1}^{n} \lambda_{i} \lambda_{j}\left(\alpha_{i}, Q_{1} \alpha_{j}\right)\left[\left(\beta_{i}, Q_{2} \beta_{j}\right)-\delta_{i j}\left(\Psi, Q_{2} \Psi\right)\right]\right| \\
& +\left[\varepsilon^{\prime}+2\left(\varepsilon^{\prime}\right)^{1 / 2}\right]\left\|Q_{1}\right\|\left\|Q_{2}\right\|
\end{aligned}
$$

For each $i \in I_{n}$ let $U_{i}$ be the partial isometry on $\mathrm{H}\left(J^{\mathrm{c}}\right)$ defined by

$$
U_{i} \phi=\left(\beta_{1}, \phi\right) \beta_{i}
$$

Then

$$
\begin{aligned}
& U_{i} \beta_{j}=\delta_{1 j} \beta_{i} \\
& U_{i}^{*} \beta_{j}=\delta_{1 j} \beta_{1}
\end{aligned}
$$

Since the union of $\mathrm{R}(K-J)$ over all finite $K \supset J$ is irreducible in $\mathrm{R}\left(J^{\mathrm{c}}\right)$, it follows from the density theorems of von Neumann and Kaplanski ${ }^{33)}$

32) Lemma 4.12 is a cluster property similar to lemma 6 of H. Araki, Prog. Theo. Phys. 32, 956 (1964). It should be noted that lemma 4.12 is a special case applying to type $I$ factorizations, and the uniformity in $Q_{1}, Q_{2}$ does not follow from lemma 6 of this reference.

33) See J. Dixmier (reference 15), Sec. I. 3. We use the fact that if $\mathfrak{A}, \mathfrak{B}$ are *-algebras, $\mathfrak{A} \subset \mathfrak{B}$ and $\mathfrak{A}$ is strongly dense in $\mathfrak{B}$ then the unit sphere of hermitean elements of $\mathfrak{A}$ is strongly dense in the unit sphere of hermitean elements of $\mathfrak{B}$. 
that there exists a finite $K \supset J$ and operators $V_{i} \in \mathrm{R}(K-J),\left\|V_{i}\right\| \leq 1, i \in I_{n}$ such that

$$
\begin{aligned}
& \left\|\left(U_{i}-V_{i}\right) \beta_{1}\right\|<\delta \\
& \left\|\left(U_{i}^{*}-V_{i}^{*}\right) \beta_{j}\right\|<\delta
\end{aligned}
$$

for all $i, j \in I_{n}$ where

$$
\delta=\varepsilon^{\prime} / \lambda(n)
$$

where

$$
\lambda(n)=\sup \left\{1, \sum_{\substack{i, j=1 \\ i \neq j}}^{n} \lambda_{i} \lambda_{j}\right\}
$$

It follows that

$$
\left\|\beta_{i}-V_{i} \beta_{1}\right\|=\left\|\left(U_{i}-V_{i}\right) \beta_{1}\right\|<\delta
$$

We have, for $Q_{2} \in \mathrm{R}\left(K^{\mathrm{c}}\right)$,

$$
\begin{aligned}
& \left|\left(\beta_{i}, Q_{2} \beta_{j}\right)-\delta_{i j}\left(\beta_{1}, Q_{2} \beta_{1}\right)\right| \\
& \quad<\left|\left(\beta_{1}, Q_{2}\left[V_{i}^{*} V_{j}-\delta_{i j}\right] \beta_{1}\right)\right|+2 \delta|| Q_{2}||
\end{aligned}
$$

Since

$$
V_{i}^{*} V_{j} \beta_{1}=\delta_{i j} \beta_{1}+\left(V_{i}^{*}-U_{i}^{*}\right) \beta_{j}+V_{i}^{*}\left(V_{j}-U_{j}\right) \beta_{1}
$$

we get

$$
\left|\left(\beta_{i}, Q_{2} \beta_{j}\right)-\delta_{i j}\left(\beta_{1}, Q_{2} \beta_{1}\right)\right|<4 \delta\left\|Q_{2}\right\|
$$

for all $i \in I_{n}$. Thus we find,

$$
\begin{aligned}
& \left|\sum_{\substack{i, j=1 \\
i \neq j}}^{n} \lambda_{i} \lambda_{j}\left(\alpha_{i}, Q_{1} \alpha_{j}\right)\left(\beta_{i}, Q_{2} \beta_{j}\right)\right| \\
& \quad<\lambda(n)\left\|Q_{1}\right\| 4 \delta\left\|Q_{2}\right\| \\
& \quad \leq 4 \varepsilon^{\prime}\left\|Q_{1}\right\| \| Q_{2} \mid
\end{aligned}
$$

Using Eqs. (4.111) and (4.114) we have

$$
\begin{aligned}
& \left|\left(\Psi, Q_{2} \Psi\right)-\left(\beta_{1}, Q_{2} \beta_{1}\right)\right| \\
& \quad<\left|\sum_{i=1}^{n} \lambda_{i}^{2}\left[\left(\beta_{i}, Q_{2} \beta_{i}\right)-\left(\beta_{1}, Q_{2} \beta_{1}\right)\right]\right|+2 \varepsilon^{\prime}|| Q_{2}||
\end{aligned}
$$

Since $\sum_{i=1}^{n} \lambda_{i}^{2} \leq 1$, it follows from Eq. (4. 126) that 


$$
\left|\left(\Psi, Q_{2} \Psi\right)-\left(\beta_{1}, Q_{2} \beta_{1}\right)\right|<\left(4 \delta+2 \varepsilon^{\prime}\right)|| Q_{2}||
$$

Using Eq. (4.126) again, we have

$$
\begin{aligned}
\left|\left(\Psi, Q_{2} \Psi\right)-\left(\beta_{1}, Q_{2} \beta_{1}\right)\right| & <\left(8 \delta+2 \varepsilon^{\prime}\right)|| Q_{2} \| \\
& \leq 10 \varepsilon^{\prime}\left\|Q_{2}\right\|
\end{aligned}
$$

for all $i \in I_{n}$. It follows that

$$
\begin{aligned}
& \left|\sum_{i=1}^{n} \lambda_{i}^{2}\left(\alpha_{i}, Q_{1} \alpha_{i}\right)\left[\left(\beta_{i}, Q_{2} \beta_{i}\right)-\left(\Psi, Q_{2} \Psi\right)\right]\right| \\
& \quad<10 \varepsilon^{\prime}\left\|Q_{1}\right\|\left\|Q_{2}\right\|
\end{aligned}
$$

Using Eqs. (4.115), (4.127) and (4.131) we get

$$
\left|\left(\Psi, Q_{1} Q_{2} \Psi\right)-\left(\Psi, Q_{1} \Psi\right)\left(\Psi, Q_{2} \Psi\right)\right|<\mathrm{f}\left(\varepsilon^{\prime}\right)\left\|Q_{1}\right\|\left\|Q_{2}\right\|
$$

where

$$
f\left(\varepsilon^{\prime}\right)=15 \varepsilon^{\prime}+2\left(\varepsilon^{\prime}\right)^{1 / 2}
$$

for all $Q_{1} \in \mathrm{R}(J), Q_{2} \in \mathrm{R}\left(K^{\mathrm{c}}\right)$. Since $\varepsilon^{\prime}$ was arbitrary, we can choose $\varepsilon^{\prime}$ so that $f\left(\varepsilon^{\prime}\right)<\varepsilon$. Q.E.D.

Lemma 4. 13: Let $\left\{R_{\alpha}\right\}_{\alpha \in A}$ be a type I factorization. If there exists a unit vector $\Psi$ such that

$$
\sup _{\boldsymbol{\Sigma}} \inf _{J \subset K^{\mathrm{c}}} \mathrm{d}\left(\Psi ; \mathrm{R}(J), \mathrm{R}\left(J^{\mathrm{c}}\right)\right)=1-\delta, \quad \delta>0
$$

where $K, J$ are finite subsets, then there is some infinite subset $J \subset A$ such that $R(J)$ is not type $I$.

Proof: We assume that all $\mathrm{R}(J)$ are type $\mathrm{I}$ and derive a contradiction. We construct a countable family of mutually disjoint finite sets $J_{k}, k \in I_{\infty}$ such that for any $Q_{1} \in \bigvee_{k=1}^{n} \mathrm{R}(J), Q_{2} \in \bigvee_{k=n+1}^{\infty} \mathrm{R}\left(J_{k}\right)$ we have

$$
\left|\left(\Psi, Q_{1} Q_{2} \Psi\right)-\left(\Psi, Q_{1} \Psi\right)\left(\Psi, Q_{2} \Psi\right)\right|<\left.\varepsilon_{n}\right|_{i} Q_{1}|| \| Q_{2}||
$$

where $\lim \varepsilon_{n}=0$, and

$$
\mathrm{d}\left(\Psi ; \mathrm{R}\left(J_{k}\right), \mathrm{R}\left(J_{k}^{\mathrm{c}}\right)\right)<1-\delta / 2
$$

for all $k>1$. Choose a finite $J_{1}$ such that

$$
\mathrm{d}\left(\Psi ;\left(J_{1}\right), \mathrm{R}\left(J_{1}^{\mathrm{c}}\right)\right)<1-\delta / 2
$$


Let $\varepsilon_{n}>0$ be any sequence satisfying $\lim \varepsilon_{n}=0$. By lemma 4.12 there exists a finite $K_{1} \supset J_{1}$ such that Eq. (4.135) is satisfied for $n=1$ by any $Q_{2} \in \mathrm{R}\left(K_{1}^{\mathrm{c}}\right)$. Let $J_{2}^{\prime}$ be any finite subset of $K_{1}^{\mathrm{c}}$ such that Eq. (4.136) is satisfied. Let $K_{2} \supset K_{1} \cup J_{2}$ be any finite subset such that Eq. (4.135) is satisfied for $n=2$ by all $Q_{1} \in \mathrm{R}\left(K_{1} \cup J_{2}\right), Q_{2} \in \mathrm{R}\left(K_{2}^{\mathrm{c}}\right)$. Repetition of this process gives the desired sequence.

Let $J=\cup J_{k}$. By assumption $\mathrm{R}(J)$ is type $\mathrm{I}$ and we have

$$
\begin{aligned}
& H=\mathrm{H}(J) \otimes \mathrm{H}\left(J^{\mathrm{c}}\right) \\
& \mathrm{R}(J)=\mathrm{B}(\mathrm{H}(J)) \otimes \mathbb{1}
\end{aligned}
$$

The unit vector $\Psi \in H$ defines a density matrix $d$ on $\mathrm{H}(J)$ by Eq. (2. 4). It follows from Eq. (4.135) and lemma 4. 11 that the countable type I factorization $\left\{\mathrm{R}\left(J_{k}\right)\right\}_{k \in I_{\infty}}$ is a PTPF (take $d_{k}=d_{k}^{\prime}=d$ in lemma 4.11). By assumption all $\vee \mathrm{R}\left(J_{k}\right)$ are type $\mathrm{I}$, and lemma 4.5 implies that $\mathrm{R}\left(J_{k}\right)$ is a TPF on the space $\mathrm{H}(J)$. Thus $\left\{\mathrm{R}\left(J^{\mathrm{c}}\right), \mathrm{R}\left(J_{k}\right)\right\}$ is a TPF on the space $H$. This implies that

$$
\lim _{k \rightarrow \infty} \mathrm{d}\left(\Psi ; \mathrm{R}\left(J_{k}\right), \mathrm{R}\left(J_{k}^{\mathrm{c}}\right)\right)=1
$$

which contradicts Eq. (4.136). Q.E.D.

Lemmas 4.8 and 4.13 imply that a type I factorization is a TPF if and only if all $\mathrm{R}(J)$ are type I. This is our main result, which we summarize as

Theorem 4. 1: Let $\Re$ be a complete atomic Boolean algebra of Type I factors with atoms $\left\{R_{\alpha}\right\}_{\alpha \in A}$ on the Hilbert space $H$. Then thare exist Hilbert spaces $H_{\infty}, \alpha \in A$ such that $H$ is unitarily equivalent to the ITPS $\underset{\alpha \in A}{\otimes} H_{\infty}$ and $R_{a}$ is unitarily equivalent to

$$
\mathrm{B}\left(H_{\omega}\right) \otimes\left\{\underset{\alpha^{\prime} \neq \infty}{\otimes} \mathbb{1}_{\alpha^{\prime}}\right\}
$$

$\Re$ is determined up to unitary equivalence by the dimension function $\operatorname{dim} H_{a}$.

\section{Complete Boolean Algebras of Type I Factors and Continuous Tensor Product Spaces}

In this section we discuss complete Boolean algebras of type I factors, 
and introduce a continuous tensor product of Hilbert spaces (CTPS) which is a generalization of the discrete ITPS defined by von Neumann. ${ }^{2}$

Given a Hilbert space $K$, we define an "exponential" Hilbert space $\mathrm{e}^{K}$ with the property that any discrete direct sum decomposition of $K$ gives a discrete tensor product decomposition of $\mathrm{e}^{K}$ (Theorem 5.1). We then show that any complete Boolean algebra $\mathfrak{\beta}$ of projection operators on $K$ defines a complete Boolean algebra $\mathrm{e}^{\mathfrak{B}}$ of type $I$ factors on $\mathrm{e}^{K}$ (Theorem 5.2). This result for nonatomic $\$$ suggests the definition of a CTPS as the exponential of a direct integral space. These CTPS obey an associative rule which demonstrates explicitly the relation with the discrete ITPS of von Neumann. We define factorizable vedtors and operators relative to a complete Boolean algebra of type $I$ factors. For the structure $\left(\mathrm{e}^{K}, \mathrm{e}^{\mathfrak{B}}\right)$ where $\mathfrak{S}_{\mathfrak{B}}$ is a complete nonatomic Boolean algebra of projections we give some (possibly unbounded) factorizable linear operators on $\mathrm{e}^{K}$ (Theorem 5.3).

\section{Exponential Hilbert Space}

Let $K$ be a Hilbert space. We construct an exponential space $\mathrm{e}^{K}$ as follows. Let $(\otimes K)^{n}$ denote the $n$-fold tensor product of $K$ with itself, and $(\otimes K)_{\mathrm{S}}^{n}$ the subspace of $(\otimes K)^{n}$ spanned by the vectors $(\otimes \phi)^{n}, \phi \in K{ }^{34)}$ $(\otimes K)^{0}=(\otimes K)_{\mathrm{s}}^{0}$ is the one-dimansional Hilbert space of complex numbers. Define $^{35)}$

$$
\mathrm{e}^{K}=\bigoplus_{n=0}^{\infty}(\otimes K)_{\mathrm{s}}^{n}
$$

We note that $\mathrm{e}^{K}$ is always infinite dimensional (unless $\operatorname{dim} K=0$ ). We consider vectors in $\mathrm{e}^{K}$ of the form

34) One usually defines $(\otimes K)_{\mathrm{s}}^{n}$ as the subspace of $(\otimes K)^{n}$ spanned by all symmetrized product vectors. However our definition is more convenient for our present purposes. Since the symmetrized product of $\phi_{1} \cdots \phi_{n}$ can be obtained as $\sum\left(\Pi \varepsilon_{i}\right)\left(\otimes \sum_{i} \varepsilon_{i} \phi_{i}\right)^{n}$ where the sum is over all $\varepsilon_{i}= \pm 1$, the two definitions are equivalent.

35) This space is well known to physicists as the Hilbert space on which the Fock representation ${ }^{36)}$ of the CCRs over the space $K$ is defined. However Eqs. (5.2), (5.3) and lemma 5.1 are perhaps not so familiar.

36) V. Fock, Z. Physik 75, 622 (1932); J.M. Cook, Trans. Am. Math. Soc. 74, 222 (1953). 


$$
\mathrm{e}^{\phi}=\bigoplus_{n=0}^{\infty}(n !)^{-1 / 2}(\otimes \phi)^{n}, \quad \phi \in K
$$

We have

$$
\left(\mathrm{e}^{\phi}, \mathrm{e}^{\psi}\right)=\mathrm{e}^{(\phi, \psi)}
$$

We shall denote the vector $\mathrm{e}^{0}$ by $\Omega$. Note that $(\otimes K)_{\mathrm{S}}^{0}$ is a one-dimensional space spanned by the vector $\Omega$.

Lemma 5. 1: The vectors $\mathrm{e}^{\phi}$ are a total set in $\mathrm{e}^{K}$.

Proof: It suffices to show that the linear space spanned by the vectors $\mathrm{e}^{\phi}$ contains all $(\otimes \phi)^{n}$ for each $n$. The proof is by induction. For $n=0, \mathrm{e}^{0}=\Omega$ spans $(\otimes K)_{\mathrm{S}}^{0}$. Now assume that each $(\otimes K)_{\mathrm{S}}^{j}, j=1, \cdots, n-1$ is spanned by the vectors $\mathrm{e}^{\phi}$. Consider the vector

$$
x_{n}(\lambda)=\mathrm{e}^{\lambda \phi}-\sum_{j=0}^{u-1}(j !)^{-1 / 2}(\otimes \lambda \phi)^{j}
$$

We have

$$
\lim _{\lambda \rightarrow 0} x_{n}(\lambda) / \lambda^{n}=(n !)^{-1 / 2}(\otimes \phi)^{n}
$$

Q.E.D.

Theorem 5.1: Let $K=\oplus K_{a}$ be a discrete direct sum decomposition of $K$. Let $\otimes \mathrm{e}^{K_{a}}$ be the ITPS relative to the product vector $\mathrm{e}^{0}=\Omega=\otimes \Omega_{a}$. The mapping

$$
\mathrm{e}^{\phi} \rightarrow \otimes \mathrm{e}^{\phi_{\boldsymbol{\omega}}}
$$

defined on total sets gives a unitary operator from $\mathrm{e}^{K}$ onto $\otimes \mathrm{e}^{K_{\alpha}}$.

Proof: For any $\Psi \in K$ we have $\Psi=\oplus \Psi_{\omega}, \Psi_{\omega} \in K_{a}$ and $\sum\left\|\Psi_{a}\right\|^{2}=$ $\|\Psi\|^{2}<\infty$. Conversely, for any $\Psi_{a} \in K_{a}$ such that $\sum\left\|\Psi_{\alpha}\right\|^{2}<\infty$ we have $\otimes \mathrm{e}^{\Psi_{a}} \approx \otimes \Omega_{\alpha}$. These vectors form a total set in $\otimes \mathrm{e}^{K_{\alpha}}$. The mapping

$$
\mathrm{e}^{\Phi} \rightarrow \bigotimes_{\alpha} \mathrm{e}^{\Phi_{\alpha}}
$$

is isometric and one to one between total sets. Hence it can be extended by linearity and continuity to a unitary operator from $\mathrm{e}^{K}$ onto $\otimes \mathrm{e}^{K_{\alpha}}$. Q.E.D. 
Complete Boolean Algebras of Projections

The set of all projection operators on a Hilbert space $K$ can be given the structure of an orthocomplemented lattice as follows. If $S$ is any subset of $K$, let

$$
S^{\perp}=\{\phi \in K:(\phi, \chi)=0 \text { for all } \chi \in S\}
$$

Let $\mathrm{P}(m)$ denote the projection onto the closed linear subspace $m$. We define the lattice operations by

$$
\begin{aligned}
& \mathrm{P}\left(m_{1}\right) \vee \mathrm{P}\left(m_{2}\right)=\mathrm{P}\left(\left(m_{1} \cup m_{2}\right)^{\perp \perp}\right) \\
& \mathrm{P}\left(m_{1}\right) \wedge \mathrm{P}\left(m_{2}\right)=\mathrm{P}\left(m_{1} \cap m_{2}\right)
\end{aligned}
$$

where $U, \cap$ denote set theoretical union and intersection respectively. The orthocomplementation is defined by

$$
\mathrm{P}(m)^{\prime}=\mathrm{P}\left(m^{\perp}\right)=1-\mathrm{P}(m)
$$

A sublattice $\mathfrak{S}$ is a Boolean algebra if and only if all $P \in \mathfrak{P}$ commute. If $K$ is separable, any complete Boolean algebra $\mathfrak{s}$ of projections can be characterized up to unitary equivalence as follows. ${ }^{37}$ There is a measure space $Z$, a positive measure $\mu$ on $Z$, and a measurable field of Hilbert spaces $\mathrm{K}(z)$ such that $K$ is a direct integral

$$
K=\int^{\oplus} \mathrm{K}(z) \mathrm{d} \mu(z)
$$

Let $E$ be the complete Boolean algebra of all measurable subsets of the space $Z$, where the lattice operations $\vee, \wedge$ are given by set theoretical union and intersection respectively, and $X^{\prime}$ is the complement of the set $X$. For any $X \in E$, let $\mathrm{P}(X)$ be the projection onto all $\phi \in K$ such that $\phi(z)=0$, $z \in X^{\prime}$. Then $X \rightarrow \mathrm{P}(X)$ is a representation of $E$ by projection operators, and $\mathfrak{P}$ is the complete Boolean algebra of all $\mathrm{P}(X)$. The unitary invariants of $\mathfrak{F}$ are the equivalence class of the measure $\mu$, and the (equivalence class of) dimension function $\operatorname{dim} \mathrm{K}(z)$.

37) See, for example, J. Dixmier (reference 15), Chap. II; G.W. Mackey, Notes on Group Representations (Department of Mathematics, University of Chicago, 1955), Chapter II. 


\section{Complete Boolean Algebras of Type I Factors}

We now show that any complete Boolean algebra $\mathfrak{P}$ of projection operators on a Hilbert space $K$ gives a complete Boolean algebra $\mathrm{e}^{\mathfrak{B}}$ of type I factors on the Hilbert space $\mathrm{e}^{K}$. Let $P \in \mathfrak{S}$, then

$$
K=P K \oplus(1-P) K
$$

and by Theorem 5.1 we have

$$
\mathrm{e}^{K}=\mathrm{e}^{P K} \otimes \mathrm{e}^{(1-P) K}
$$

We define the type $\mathrm{I}$ factor $\mathrm{R}(P)=\mathrm{e}^{P}$ by

$$
\mathrm{e}^{P}=\mathrm{B}\left(\mathrm{e}^{P K}\right) \otimes \mathbb{1}
$$

If $P \neq 0, \mathrm{e}^{P}$ is type $\mathrm{I}_{\infty}$. Clearly

$$
\mathrm{e}^{P^{\prime}}=\mathrm{e}^{(1-P)}=\left(\mathrm{e}^{P}\right)^{\prime}
$$

From Theorem 5.1 one can easily prove that for any $P_{1}, P_{2} \in \mathfrak{S}$ we have

$$
\begin{aligned}
& \mathrm{e}^{P_{1}} \vee \mathrm{e}^{P_{2}}=\mathrm{e}^{P_{1} \vee P_{2}} \\
& \mathrm{e}^{P_{1}} \wedge \mathrm{e}^{P_{2}}=\mathrm{e}^{P_{1} \wedge P_{2}} .
\end{aligned}
$$

Thus the type I factors $\mathrm{e}^{P}, P \in \mathfrak{P}$ form a Boolean algebra which we denote by $\mathrm{e}^{\mathfrak{B}}$. It remains to show that $\mathrm{e}^{\mathfrak{B}}$ is complete. Let $P_{a}$ be any set of projections in $\mathfrak{S}_{3}$. We can take the $P_{a}$ to be well-ordered. Then $Q_{\infty}=$ $P_{a b}-\bigvee_{\alpha^{\prime}}<P^{\prime} P_{\alpha^{\prime}}$ is a partition of $\vee P_{a}$, and we have the direct sum decomposition

$$
K=\left(1-\vee P_{a}\right) K \oplus\left(\oplus Q_{\infty} K\right)
$$

By theorem 5.1 we have

$$
\mathrm{e}^{K}=\mathrm{e}^{\left(1-\bigvee P_{\omega}\right) K} \otimes\left(\otimes \mathrm{e}^{Q_{\omega} K}\right)
$$

It follows from von Neumann's results (see Eq. (3.30)) that

$$
\mathrm{e}^{\vee P_{\alpha}}=\vee \mathrm{e}^{Q_{\alpha}}=\vee \mathrm{e}^{P_{\alpha}}
$$

Eq. (5.14) then gives

$$
\mathrm{e}^{\wedge P_{\alpha}}=\wedge \mathrm{e}^{P_{\alpha}}
$$

Thus $\mathrm{e}^{\mathfrak{B}}$ is a complete Boolean algebra of type I factors. By construction 
it is clear that unitary equivalence of $(K, \mathfrak{P})$ and $\left(K^{\prime}, \mathfrak{P}^{\prime}\right)$ implies unitary equivalence of $\left(\mathrm{e}^{K}, \mathrm{e}^{\mathfrak{B}}\right)$ and $\left(\mathrm{e}^{K^{\prime}}, \mathrm{e}^{\mathfrak{B}^{\prime}}\right)$. We summarize these results as

Theorem 5.2: Let $K$ be a Hilbert space, $\mathfrak{P}$ a complete Boolean algebra of projection operators on $K$. For each $P \in \mathfrak{P}$ we have $\mathrm{e}^{K}=\mathrm{e}^{P K} \otimes$ $\mathrm{e}^{(1-P) K}$ and the equation

$$
\mathrm{e}^{P}=\mathrm{B}\left(\mathrm{e}^{P K}\right) \otimes 1
$$

defines a complete Boolean algebra $\mathrm{e}^{\mathfrak{B}}$ of type I factors on $\mathrm{e}^{K}$. If $(K, \mathfrak{S})$ and $\left(K^{\prime}, \mathfrak{P}^{\prime}\right)$ are unitarily equivalent, then $\left(\mathrm{e}^{K}, \mathrm{e}^{\mathfrak{B}}\right)$ and $\left(\mathrm{e}^{K^{\prime}}, \mathrm{e}^{\mathfrak{B}^{\prime}}\right)$ are unitarily equivalent.

Since $\mathrm{e}^{P}$ is always type $\mathrm{I}_{\infty}$ if $P \neq 0$, not all complete Boolean algebras of type $I$ factors can be obtained in this way. Let $\Re$ be a complete atomic Boolean algebra of type I factors with atoms $\left\{R_{a i}\right\}_{\alpha \in A}$ where each $R_{a}$ is type $I_{\infty}$. Let $\left\{K_{a}\right\}_{a \in A}$ be Hilbert spaces with $\operatorname{dim} K_{a} \neq 0$, and let $K=$ $\oplus K_{a}$. Let $P_{a}$ be the projection on $K_{a}, \mathfrak{P}$ the atomic Boolean algebra of projections generated by the $P_{a}$. Then $\mathfrak{R}=\mathrm{e}^{\mathfrak{B}}$. But the equivalence class of $\mathfrak{P}$ depends on $\operatorname{dim} K_{a}, \alpha \in A$ which is not determined by $\Re$. However, we shall prove in Sec. 6 that if $\Re$ is any complete nonatomic Boolean algebra of type I factors satisfying a certain condition, then there is a complete nonatomic Boolean algebra $\mathfrak{F}=\log \mathfrak{R}$ of projections such that $\mathfrak{R}=\mathrm{e}^{\mathfrak{P}}$, and $\Re$ determines $\mathfrak{乛}$ up to unitary equivalence.

\section{Continuous Tensor Product Spaces}

The preceding discussion of Boolean algebras of projections and type I factors suggests that we consider spaces $\mathrm{e}^{K}$ where $K$ is a direct integral. We now show how this leads to a definition of a continuous tensor product space (CTPS).

To further motivate our definition, let $H=\otimes H_{i}$ be the I'TPS of the Hilbert spaces $H_{i}$ relative to some unit product vector $\Omega$. Then we can write

$$
\Omega=\otimes \Omega_{i}, \quad\left\|\Omega_{i}\right\|=1
$$

Now let $\Phi$ be any product vector with $(\Omega, \Phi)=1$. Note that these vectors 
form a total set in $H$. We can write

$$
\Phi=\otimes \Phi_{\imath} \quad \text { where } \quad\left(\Omega_{i}, \Phi_{\imath}\right)=1
$$

Then

$$
\Phi=\Phi_{i}-\Omega_{i}
$$

is orthogonal to $\Omega_{i}$. Thus we have

$$
\Phi=\otimes\left[\Omega_{i}+\Phi_{i}^{\prime}\right]
$$

and

$$
\|\Phi\|^{2}=\Pi\left[1+\left\|\Phi_{i}^{\prime}\right\|^{2}\right]=\mathrm{e}^{\Sigma \log \left(1+\left\|\Phi_{i}^{\prime}\right\|^{2}\right.}
$$

We complete our motivation by noting that continuous infinite products of this form have the following simple property. Let $\mathrm{f}(x)$ be any integrable function on $[0,1]$. Divide the interval into $n$ subsets $R_{j}$ with Lebesgue measure $\Delta_{j}$. Let $x_{j} \in R_{j}$, then

$$
\prod_{j=1}^{n}\left[1+\mathrm{f}\left(x_{j}\right) \Delta_{j}\right]=\exp \left\{\sum_{j=1}^{n} \log \left[1+\mathrm{f}\left(x_{j}\right) \Delta_{j}\right]\right\}
$$

Since

$$
\log (1+x)=x-\frac{1}{2} x^{2}+\cdots
$$

we have

$$
\lim _{\substack{n \rightarrow \infty \\ \Delta_{j} \rightarrow 0}} \prod_{j=1}^{n}\left[1+\mathrm{f}\left(x_{j}\right) \Delta_{j}\right]=\exp \left\{\int_{0}^{1} \mathrm{f}(x) \mathrm{d} x\right\}
$$

These considerations suggest the following definition of a CTPS.

Let $Z$ be a measure space, and $\mu$ a non-atomic positive measure on $Z$ (we want $\mu(\{z\})=0$ so that only the leading term in Eq. (5.23) contributes). Let $z \rightarrow \mathrm{H}(z), z \in Z$ be a field of complex Hilbert spaces on $Z$ such that the function $\operatorname{dim} \mathrm{H}(z)$ is measurable. Choose some fixed vector field $\Omega(z) \in \mathrm{H}(z)$ with $\|\Omega(z)\|=1$. We shall refer to $\Omega(z)$ as the reference product vector. Let $\mathrm{K}(z)$ be the subspace of $\mathrm{H}(z)$ orthogonal to $\Omega(z)$, and let

$$
K=\int^{\oplus} \mathrm{K}(z) \mathrm{d} \mu(z)
$$

be the direct integral of the $\mathrm{K}(z){ }^{37)}$ We now define the continuous tensor product of the $H(z)$, relative to the reference vector $\Omega(z)$, as the 
exponential space $\mathrm{e}^{K}$. Thus

$$
\underset{(z, \mu)}{\otimes} \mathrm{H}(z)=\mathrm{e}^{K}
$$

In the following we shall use whichever of these notations seems more appropriate. We shall not explicityly indicate the dependence on $\Omega(z)$ unless necessary.

We now extend the definition of a product vector as follows.

Definition 5.1: Let $K$ be a Hilbert space, $\mathfrak{P}$ a complete nonatomic Boolean algebra of projections on $K . \quad A$ vector $\Psi \in \mathrm{e}^{K}$ is called a product vector (relative to $\mathrm{e}^{\mathfrak{S}}$ ) if it is of the form $C \mathrm{e}^{\phi}$ where $\phi \in K$ and $C$ is a complex number.

For product vectors in a CTPS we can use the following symbolic notation, which is the analog of Eqs. (5.17-20) for the ITPS of von Neumann. Given $\Omega(z)$ we consider vector fields $\Phi(z)$ such that $(\Omega(z), \Phi(z))$ $=1$. Then $\Phi^{\prime}(z)=\Phi(z)-\Omega(z)$ is in the subspace $\mathrm{K}(z)$ orthogonal to $\Omega(z)$. Conversely any $\Phi^{\prime}(z) \in \mathrm{K}(z)$ gives such a $\Phi(z)$. This suggests the notation

$$
\underset{(z, \mu)}{\otimes}\left[\Omega(z)+\Phi^{\prime}(z) \mathrm{d} \mu(z)^{1 / 2}\right]
$$

for product vectors of the form $\mathrm{e}^{\Phi}, \Phi(z)=\int \Phi^{\prime}(z) \mathrm{d} \mu(z)^{1 / 2}$.

It follows from Theorem 5.1 that these CTPS obey a restricted associative law, analogous to that for the ITPS of von Neumann. Let $Z_{i}$ be a partition of the Boolean algebra of all measurable subsets of the space $Z$, such that $\mu\left(Z_{i}\right)>0$. Let

$$
\mathrm{H}\left(Z_{i}\right)=\underset{\left(Z_{i}, \mu\right)}{\otimes} \mathrm{H}(z)
$$

be the CTPS of the $\mathrm{H}(z)$ over the measure space $\left(Z_{i}, \mu\right)$ with respect to the reference vector $\Omega(z)$, and let

$$
\Omega_{i}=\underset{\left(z_{i}, \mu\right)}{\otimes} \Omega(z)
$$

Let

$$
H=\otimes^{\left(\otimes \Omega_{i}\right)} \mathrm{H}\left(Z_{i}\right)
$$

be the ITPS of the $\mathrm{H}\left(Z_{i}\right)$. Then $H$ and $\underset{(Z, \mu)}{\otimes} \mathrm{H}(z)$ are isomorphic under 
the mapping

$$
\underset{(Z, \mu)}{\otimes}\left\{\Omega(z)+\Phi^{\prime}(z) \mathrm{d} \mu(z)^{1 / 2}\right\} \rightarrow \underset{i}{\otimes}\left\{\underset{\left(Z_{i}, \mu\right)^{\prime}}{\otimes}\left[\Omega(z)+\Phi^{\prime}(z) \mathrm{d} \mu(z)^{1 / 2}\right]\right\}
$$

\section{Generalized Tensor Products}

We now consider the general case where the measure $\mu$ may have a discrete part. The measure may then be decomposed into a continuous non-atomic measure $\mu_{\mathrm{c}}$ and a discrete measure $\mu_{\mathrm{d}}$

$$
\mu=\mu_{\mathrm{c}}+\mu_{\mathrm{d}}
$$

Let $Z_{\mathrm{d}}=\left\{z_{1}, \cdots\right\}$ be the set of atoms of the measure $\mu_{\mathrm{d}}$. Let $\Omega\left(z_{j}\right)$, $z_{j} \in Z_{\mathrm{d}}$ be some sequence of unit vedtors and let

$$
H_{\mathrm{d}}=\underset{z_{j} \in Z_{\mathrm{d}}}{\otimes} \mathrm{H}\left(z_{j}\right)
$$

be the ITPS relative to the product vector $\otimes \Omega\left(z_{j}\right)$. For the continuous part $\mu_{\mathrm{c}}$, let $H_{\mathrm{c}}$ be the CTPS defined above. Thus the general definition of

$$
H=\underset{(z, \mu)}{\otimes} \mathrm{H}(z)
$$

is given by

$$
H=H_{\mathrm{c}} \otimes H_{\mathrm{d}}
$$

Factorizable Vectors and Operators

In Sec. 2 we defined factorizable vectors for atomic Boolean algebras of type I factors (definition 2.3). We now extend this definition to the general case.

Definition 5.2: Let $H$ be a Hilbert space and let $\Re$ be a Boolean algebra of type $I$ factors on $H$. A vector $\Psi \in H$ is called factorizable (relative to $\Re$ ) if for all $Q_{i} \in R_{i}$ we have

$$
\left(\Psi, \prod_{i=1}^{n} Q_{i} \Psi\right)=(\Psi, \Psi)^{1-n} \prod_{i=1}^{n}\left(\Psi, Q_{i} \Psi\right)
$$

for all finite partitions $\left\{R_{i}\right\}$. An operator $Q \in \mathrm{B}(H)$ is called factorizable if for all finite partitions $R_{i} \in \Re, i \in I_{n}$ of $\mathrm{B}(H)$ we have 


$$
Q=\prod_{i=1}^{n} Q_{i}, \quad Q_{i} \in R_{i}
$$

If $\Re$ is a complete Boolean algebra of type $I$ factors, and $\left\{R_{\alpha}\right\}$ is any partition of $\mathrm{B}(H)$, then the $R_{\omega}$ are the atoms of a complete atomic Boolean algebra of type I factors. By theorem 4.1 we have $H=\otimes H_{a}$. It follows from lemma 3.2 that the preceding definition of a factorizable vector $\Psi$ is equivalent to requiring that

$$
\Psi=\otimes \Psi_{\alpha}
$$

for all partitions $\left\{R_{a}\right\}$. It is this form of the definition that we shall use in the following.

We now consider the structure $\left(\mathrm{e}^{K}, \mathrm{e}^{\mathfrak{B}}\right)$ where $\mathfrak{S}$ is a complete nonatomic Boolean algebra of projections. Clearly any product vector $C \mathrm{e}^{\phi}, \phi \in K$ (see definition 5.1) is factorizable. It is a rather obvious conjecture that the only factorizable vectors are product vectors. To prove this we must be able to reconstruct $(K, \mathfrak{P})$ from $\left(\mathrm{e}^{K}, \mathrm{e}^{\mathfrak{P}}\right)$. This is done is Sec. 6.

We now define some factorizable operators for the structure $\left(\mathrm{e}^{K}, \mathrm{e}^{\Re}\right)$. These operators are to be considered as unbounded operators defined on the dense subspace $H_{0}$ of finite linear combinations of product vectors (unless stated otherwise). For any $Q \in \mathrm{B}(K)$ we define a linear operator $\mathrm{S}(Q)$ on $H_{0}$ by

$$
\mathrm{S}(Q) \mathrm{e}^{\phi}=\mathrm{e}^{Q \phi}
$$

or equivalently

$$
\mathrm{S}(Q)=\bigoplus_{n=0}^{\infty}(\otimes Q)^{n}
$$

(see Eq. (5.1)). $S(0)$ is the one-dimensional projection operator onto the vector $\Omega=\mathrm{e}^{0}$, and $\mathrm{S}(1)$ is the identity operator on $\mathrm{e}^{K}$. If $\|Q\| \leqslant 1$ then $\mathrm{S}(Q)$ is bounded. If $\|Q\|>1$ then $\mathrm{S}(Q)$ is unbounded. Let

$$
\mathfrak{U}=\{P \in \mathfrak{P}\}^{\prime \prime}
$$

We now show that $\mathrm{S}(Q)$ is factorizable if $Q \in \mathfrak{Q}^{\prime}$.

Thus let $P_{a} \in \mathfrak{P}$ be any finite partition of the identity, then

$$
K=\oplus K_{a^{i}},
$$




$$
Q=\oplus Q_{\infty}
$$

where $K_{\omega}=P_{\alpha} K$, and $Q_{\alpha}=P_{\alpha} Q$.

By lemma 5.1 we have

$$
\mathrm{e}^{K}=\otimes \mathrm{e}^{K_{\alpha}}
$$

It follows from the mapping given in lemma 5.1 that

$$
\mathrm{S}(Q)=\otimes \mathrm{S}\left(Q_{\infty}\right)
$$

which proves that $\mathrm{S}(Q), Q \in \mathfrak{Q}^{\prime}$ is factorizable. If $K$ is separable then we have the direct integral decomposition ${ }^{37)}$

$$
K=\int^{\oplus} \mathrm{K}(z) \mathrm{d} \mu(z)
$$

and $Q \in \mathfrak{Y}^{\prime}$ means $Q$ is decomposable ${ }^{38}$, that is

$$
Q=\int^{\oplus} \mathrm{Q}(z) \mathrm{d} \mu(z)
$$

For any $\Psi \in K$ we define a linear operator $\mathrm{T}(\Psi)$ on $H_{0}$ by

$$
\mathrm{T}(\Psi) \mathrm{e}^{\phi}=\mathrm{e}^{(\Psi, \phi)} \mathrm{e}^{\phi}
$$

where $\Omega=\mathrm{e}^{0}$. It follows from Eq. (5.3) that on $H_{0}$ the adjoint is given by

$$
\mathrm{T}(\Psi)^{*} \mathrm{e}^{\phi}=\mathrm{e}^{\Psi+\phi}
$$

Under the decompositions given by Eqs. (5.44) and (5.46) we have $\Psi=$ $\oplus \Psi_{\infty}$ and

$$
\mathrm{T}(\Psi)=\otimes \mathrm{T}\left(\Psi_{\star}\right)
$$

It follows that any operator of the form

$$
\mathrm{T}(\Psi, Q, \chi)=\mathrm{T}(\Psi)^{*} \mathrm{~S}(Q) \mathrm{T}(\chi)
$$

where, $\Psi, \chi \in K, Q \in \mathfrak{H}^{\prime}$ is factorizable. On product vectors $\mathrm{e}^{\phi}$ we have

$$
\mathrm{T}(\Psi, Q, \chi) \mathrm{e}^{\phi}=\mathrm{e}^{(x, \phi)} \mathrm{e}^{Q \phi+\Psi}
$$

Using the symbolic notation

$$
\mathrm{e}^{\Phi}=\underset{(Z, \mu)}{\otimes}\left\{\Omega(z)+\Phi^{\prime}(z) \mathrm{d} \mu(z)^{1 / 2}\right\}
$$

38) See J. Dixmier (reference 15), p. 159. 
introduced in Eq. (5.27) we can write

$$
\begin{gathered}
\mathrm{T}(\psi, Q, \chi)=\bigotimes_{(z, \mu)}\left[1+\left(\psi(z) \Omega(z)^{*}\right) \mathrm{d} \mu(z)^{1 / 2}\right][1+Q(z)] \\
{\left[1+\left(\Omega(z) \chi(z)^{*}\right) \mathrm{d} \mu(z)^{1 / 2}\right]}
\end{gathered}
$$

where $\psi(z) \Omega(z)^{*}$ is the operator from the one-dimensional subspace of $\mathrm{H}(z)$ spanned by $\Omega(z)$ to its orthogonal complement $\mathrm{K}(z)$, defined by

$$
\left[\psi(z) \Omega(z)^{*}\right] \Omega(z)=\psi(z)
$$

and $\Omega(z) \psi(z)^{*}$ is the adjoint operator

$$
\left[\Omega(z) \psi(z)^{*}\right] \phi(z)=(\psi(z), \phi(z)) \Omega(z)
$$

The computation of the norm of $\mathrm{T}(\Psi, Q, \chi)$ is most conveniently done by using the annihilation and creation operators for the Schrodinger representation of the commutation relations. Since these operators are not used eleswhere in this paper, this calculation is given in appendix 1 . We now summarize the above as

Theorem 5. 3: Let $K$ be a Hilbert space, $\mathfrak{P}$ a complete Boolean algebra of projections on $K$. Let $H_{0}$ be the dense subspace of $\mathrm{e}^{K}$ of finite linear combination of product vectors $\mathrm{e}^{\phi}$. Let $\mathfrak{A}=\{P \in \mathfrak{P}\}^{\prime \prime}$. The equation

$$
\mathrm{T}(\Psi, Q, \chi) \mathrm{e}^{\phi}=\mathrm{e}^{(\boldsymbol{x}, \phi)} \mathrm{e}^{Q \phi+\Psi}
$$

where $\Psi, \chi \in K$ and $Q \in \mathfrak{Q}^{\prime}$ defines a linear factorizable operator on $H_{0}$. If $\|Q\|>1$ then $T(\Psi, Q, \chi)$ is unbounded. If $\|Q\| \leqslant 1$ then

$$
\|\mathrm{T}(\Psi, Q, \chi)\|^{2}=\left(\exp \|\Psi\|^{2}\right)\left(\exp \left\|\left(1-Q^{*} Q\right)^{-1 / 2}\left(\chi+Q^{*} \Psi\right)\right\|^{2}\right)
$$

if $\chi+Q^{*} \Psi$ is in the domain of $\left(1-Q^{*} Q\right)^{-1 / 2}$, otherwise $T(\Psi, Q, \chi)$ is unbounded.

\section{Nonatomic Complete Boolean Algebras of Type I Factors}

In this section we consider the problem of showing that any nonatomic complete Boolean algebra of type I factors $(H, \mathfrak{R})$ is of the form $\left(\mathrm{e}^{K}, \mathrm{e}^{\mathfrak{P}}\right)$ where $\mathfrak{P}$ is a nonatomic complete Boolean algebra of projections on the Hilbert space $K$.

Let $\left\{z_{\alpha}\right\}$ be a partition of $z$ in the lattice $\mathfrak{R}$. We shall denote such a 
partition by $B=\left\{z_{\alpha}\right\}$. If $B^{\prime}=\left\{z_{\alpha \beta}\right\}$ is a subpartition (that is for each $\alpha$, $z_{a \beta \beta}$ is a partition of $z_{a j}$ ), we write $B^{\prime}<B$. The main tool of our argument is to note that any partition $B=\left\{z_{\alpha}\right\}$ gives a complete atomic Boolean algebra of type I factors, and Theorem 4.1 implies that we can write $\mathrm{H}(z)$ as an ITPS $\otimes_{\alpha} \mathrm{H}\left(z_{a}\right)$ where $z=\vee z_{a}$.

We begin by discussing the condition that the factorizable vectors form a total set (Lemmas 6.1-3). In the following lemmas we assume this condition, and construct $\left(\mathrm{e}^{K}, \mathrm{e}^{\mathfrak{S}}\right)$. Lemma 6.4 proves that no two factorizable vectors are orthogonal, which allows us to restrict our attention to certain factorizable vectors which we prove to be of the form $\mathrm{e}^{\mathrm{f}}, f \in K$, by explicitly constructing $K$ as a Hilbert space. We then define the lattice $\mathfrak{P}=\log \Re$ in $K$. We summarize the results in Theorem 6.1. Finally we prove that any factorizable vector and any factorizable bounded linear operator can always be written in the way indicated at the end of Sec. 5 (Theorem 6.2).

We generalize definition 2.4 as follows

Definition 6.1: Let $(H, \Re)$ be a complete nonatomic Boolean algebra of type I factors. Let $B=\left\{z_{\infty}\right\}, z_{\infty} \in \Re$ be a partition of $B(H)$. For any $\Psi \in H$ we define

$$
\begin{aligned}
& \mathrm{d}(\Psi ; B)=\sup _{P_{\alpha}}\left(\Psi, \Pi_{\alpha} P_{\alpha} \Psi\right) \\
& \left(\Psi, \Pi_{\alpha} P_{\alpha} \Psi\right) \equiv \inf _{n,\left\{\alpha_{i}\right\}}\left(\Psi, \prod_{i=1}^{n} P_{\alpha_{i}} \Psi\right)
\end{aligned}
$$

where $P_{a}$ runs over all minimal projections in $z_{\infty}$.

Our construction is based on the assumption that the factorizable vectors form a total set. In Lemma 6.3 we show that this is equivalent to requiring that

$$
\mathrm{d}(\Psi ; \Re)=\inf _{B} \mathrm{~d}(\Psi ; B)>0
$$

where $B$ runs over all finite partitions of $\mathrm{B}(H)$ in the lattice $\Re$. At the moment we are unable to prove that this condition must hold for any complete nonatomic Boolean algebra of type I factors. The following lemma is intended to make this additional assumption appear reasonable.

Lemma 6.1: Let $(H, \Re)$ be a complete nonatomic Boolean algebra of 
type I factors. Let $B=\left\{z_{i}\right\}, z_{i} \in \Re$ be a countable partition of $\mathrm{B}(H)$. By Theorem 4.1

$$
H=\otimes^{\left(\otimes x_{i}\right)} \mathrm{H}\left(z_{i}\right)
$$

Let $\otimes \Psi_{i} \in H . \quad$ Then $\sum_{i}\left(1-\delta_{i}\right)<\infty$ for $\delta_{i}=\inf _{B_{i}} \mathrm{~d}\left(\Psi_{i} ; B_{i}\right)$. In particular, $\delta_{i}$ can be 0 only for a finite number of $i$.

Proof: Let $\varepsilon_{i}$ be any sequence such that

$$
0 \leq \varepsilon_{i} \leq 1-\delta_{i}, \varepsilon_{i}>0 \text { unless } \delta_{i}=1 \text {, and }
$$

$\sum \varepsilon_{i}<\infty$. Then there exist finite partitions $B_{i}=\left\{z_{i j}\right\}$ of $z_{i}$ such that

$$
\mathrm{d}\left(\Psi_{i} ; B_{i}\right) \leq \delta_{i}+\varepsilon_{i}
$$

Let $B=\left\{z_{i j}\right\}$ be the joint partition of $\mathrm{B}(H)$. Then by Theorem 4.1 we have

$$
H=\otimes_{i, j} \mathrm{H}\left(z_{i j}\right)=\otimes_{i}\left\{\otimes_{j} \mathrm{H}\left(z_{i j}\right)\right\}
$$

Since the product vectors form a total set in $H$ we must have

$$
\sup _{P_{i j}}\left(\Psi, \Pi P_{i j} \Psi\right)>0
$$

where $P_{i j}$ runs over all minimal projections in $z_{i j}$. Thus

$$
\mathrm{d}\left(\otimes \Psi_{i} ; B\right)=\Pi_{i} \mathrm{~d}\left(\Psi_{i} ; B_{i}\right)>0
$$

Hence

$$
\Pi_{i}\left(\delta_{i}+\varepsilon_{i}\right)>0
$$

which implies that ${ }^{23}$

$$
\sum_{i}\left\{1-\left(\delta_{i}+\varepsilon_{i}\right)\right\}<\infty
$$

Since $\sum \varepsilon_{i}<\infty$ we get

$$
\sum_{i}\left(1-\delta_{i}\right)<\infty
$$

which implies that at most a finite number of $\delta_{i}=0$. Q.E.D.

Lemma 6. 2: Let $S$ be a subset of the unit sphere in Hilbert space, $S^{(\mathrm{w})}$ its weak closure. Any $\Phi \in S^{(\mathrm{w})}$ is the weak limit of a sequence of vectors in $S$.

Proof: Let $\Phi \in S^{(\mathrm{w})}$, then $0 \in\{S-\Phi\}^{(\mathrm{w})}$. Hence we may assume $\Phi=0$ without loss of generality. Let $\Phi_{1} \in S$. Because $0 \in S^{(\mathrm{w})}$, there exists 
a $\Phi_{2} \in S$ such that $\left|\left(\Phi_{1}^{\prime}, \Phi_{2}\right)\right|<2^{-2}$ where $\Phi_{1}^{\prime}=\Phi_{1} /\left\|\Phi_{1}\right\|$. Similarly, we obtain a sequence $\Phi_{n} \in S$ such that $\left|\left(\Phi_{m}^{\prime}, \Phi_{n}\right)\right|<2^{-n} / n-1$ for all $m<n$, where, by Schmidt orthogonalization,

$$
\Phi_{n}=\sum_{m=1}^{n} C_{n m} \Phi_{m}^{\prime}
$$

and $\Phi_{m}^{\prime}$ is orthonormal. Now $\left|C_{n n}\right|^{2}<2$, and $\sum_{m<n}\left|C_{n m}\right|^{2}<2^{-n}$. It follows that $\Phi_{n} \rightarrow 0$ weakly. Q.E.D.

Lemma 6.3: If $\inf _{B} \mathrm{~d}(\Psi ; B)>0$ for all $\Psi \in H$, where $B$ is finite, then the factorizable vectors are a total set in $H$.

Proof: Let $\Psi \in H$. We prove that there exists a factorizable vector $\Phi$ such that $(\Phi, \Psi) \neq 0$. For each finite partition $B=\left\{z_{i}\right\}$ of $\mathrm{B}(H)$ choose a minimal projection $\mathrm{P}_{i}(B) \in z_{i}$ such that

$$
(\Psi, \mathrm{P}(B) \Psi)>\frac{1}{2} \mathrm{~d}(\Psi ; B)
$$

where $\mathrm{P}(B)=\Pi_{i} \mathrm{P}_{i}(B)$. Let $\mathrm{S}(B)=\left\{\bigcup_{B^{\prime}<B} \mathrm{P}\left(B^{\prime}\right) \Psi\right\}, S=\bigcap_{B} \mathrm{~S}(B)^{(\mathrm{w})}$. Since the intersection over any finite number of $B$ 's is non-empty, and the unit sphere in Hilbert space is weakly compact, the set $S$ is non-empty. Let $\Phi \in S$, then

$$
(\Phi, \Psi) \geq \frac{1}{2} \inf _{B} \mathrm{~d}(\Psi ; B)>0
$$

To prove that $\Phi$ is factorizable consider a partition $B=\left\{z_{i}\right\}$. Since $\Phi \in$ $\mathrm{S}(B)^{(\mathrm{w})}$, it follows from lemma 6.2 that there exists a sequence of partitions $B_{n}<B$ such that

$$
\Phi=\text { weak } \lim _{n \rightarrow \infty} \mathrm{P}\left(B_{n}\right) \Psi
$$

Each $\mathrm{P}\left(B_{n}\right) \Psi$ is a product vector in $\otimes \mathrm{H}\left(z_{i}\right)$. It now follows from lemma 3.3 that $\Phi$ is a product vector $\otimes \Phi\left(z_{i}\right)$. Since $B$ was arbitrary, $\Phi$ is factorizable. Q.E.D.

Lemma 6. 4: If $\Phi_{1}, \Phi_{2}$ are factorizable vectors, then $\left(\Phi_{1}, \Phi_{2}\right) \neq 0$.

Proof: Let $\left\|\Phi_{1}\right\|=\left\|\Phi_{2}\right\|=1$. For any partition $B=\left\{z_{i}\right\}$ of $\mathrm{B}(H)$ we have 


$$
\Phi_{j}=\bigotimes_{i} \Phi_{j}\left(z_{i}\right), \quad\left\|\Phi_{j}\left(z_{i}\right)\right\|=1, \quad j=1,2
$$

where the unit rays $e^{i \omega} \Phi_{j}(z)$ depend only on $z$, not on the partition $B$. Assume there is some $x$ such that

$$
\left(\Phi_{1}(x), \Phi_{2}(x)\right) \neq 0 .
$$

Then $x>y$ implies that $\left(\Phi_{1}(y), \Phi_{2}(y)\right) \neq 0 . \quad$ Let $x_{\omega} \in \Re$ be any well ordered set satisfying $x_{\alpha}>x, x_{\infty}>x_{\alpha^{\prime}}$ for, $\alpha>\alpha^{\prime}$ and $\left(\Phi_{1}\left(x_{\alpha i}\right), \Phi_{2}\left(x_{\alpha}\right)\right) \neq 0$. Let $y=\vee x_{\infty}$, and consider the partition of $y$ into the disjoint union of $y_{\infty}=x_{\infty}$ $\left(\underset{\alpha^{\prime}<\alpha}{\vee} x_{\alpha^{\prime}}\right)$. Then the $y_{a}$ give a complete atomic Boolean algebra of type I factors, and by Theorem 4.1 we have

$$
H(y)=\otimes H\left(y_{\alpha}\right)
$$

Since $\Phi_{1}, \Phi_{2}$ are factorizable we have

$$
\Phi_{j}(y)=\otimes \Phi_{j}\left(y_{w}\right), \quad j=1,2
$$

Since $\left(\Phi_{1}\left(y_{w}\right), \Phi_{2}\left(y_{w}\right)\right) \neq 0$, we have $\left(\Phi_{1}(y), \Phi_{2}(y)\right) \neq 0$ (see Eq. (3.8)). It now follows from Zorn's lemma that $x$ is contained in some maximal $x_{0}$ such that $y>x_{0}$ implies $\left(\Phi_{1}(y), \Phi_{2}(y)\right)=0$. If there is no $x$ satisfying Eq. (6.7) let $x_{0}=1$.

In either case $y>x_{0}$ implies

$$
\left(\Phi_{1}(y), \Phi_{2}(y)\right)=0
$$

which implies,

$$
\left(\Phi_{1}\left(y-x_{0}\right), \Phi_{2}\left(y-x_{0}\right)\right)=0
$$

Since $\mathfrak{R}$ is nonatomic, we can construct a countably infinite partition $\left\{y_{i}\right\}$ of $y-x_{0}$. By Theorem 4.1 we have

$$
\begin{aligned}
& \mathrm{H}\left(y-x_{0}\right)=\otimes \mathrm{H}\left(y_{i}\right) \\
& \Phi_{j}\left(y-x_{0}\right)=\otimes \Phi_{j}\left(y_{i}\right)
\end{aligned}
$$

But

$$
\left(\Phi_{1}\left(y_{i}\right), \Phi_{2}\left(y_{i}\right)\right)=0
$$

for all $i$ which is not possible in an ITPS for $\Phi_{j}\left(y-x_{0}\right) \neq 0$ (see Eq. (3.6)). Thus we must have $x_{0}=\mathrm{B}(H)$ and $\left(\Phi_{1}, \Phi_{2}\right) \neq 0$. Q.E.D. 
Let $\Phi$ be a factorizable vector, and $\mathrm{P}(\Phi)$ be the projection operator on the one-dimensional subspace spanned by $\Phi$. For any partition $B$ we have

$$
\mathrm{P}(\Phi)=\Pi_{i} \mathrm{P}\left(\Phi ; z_{i}\right)
$$

where $\mathrm{P}\left(\Phi ; z_{i}\right)$ is a minimal projection in $z_{\imath}$. If $\Psi$ is any other factorizable vector we have

$$
\begin{aligned}
\operatorname{Tr}\{\mathrm{P}(\Phi) \mathrm{P}(\Psi)\} & =|(\Phi, \Psi)|^{2} /\left\{\|\Phi\|^{2}\|\Psi\|^{2}\right\} \\
& =\Pi_{i} \operatorname{Tr}\left\{\mathrm{P}\left(\Phi ; z_{i}\right) \mathrm{P}\left(\Psi ; z_{i}\right)\right\}
\end{aligned}
$$

For $x \in \Re$ let

$$
\mathrm{d}(\Phi, \Psi ; x)=1-\operatorname{Tr}\{\mathrm{P}(\Phi ; x) \mathrm{P}(\Psi ; x)\}
$$

Lemma 6. 5: Let $\Phi, \Psi$ be factorizable vectors, $B=\left\{z_{\alpha}\right\}$ a partition. For any $\varepsilon>0$, there exists a subpartition $B^{\prime}=\left\{z_{\infty \beta}\right\}$ such that

$$
\mathrm{d}\left(\Phi, \Psi ; z_{a \beta \beta}\right)<\varepsilon
$$

and

$$
\sum_{\omega, \beta} \mathrm{d}\left(\Phi, \Psi ; z_{\alpha \beta \beta}\right)^{2}<\varepsilon
$$

If $B$ is a finite partition, then $B^{\prime}$ can also be chosen finite.

Proof: Let $\varepsilon^{\prime}$ be the smaller of $\varepsilon$ and $-\varepsilon / \log \{\operatorname{Tr}[\mathrm{P}(\Phi) \mathrm{P}(\Psi)]\}$. Let $z_{0}$ be the union of all $x$ with

$$
\mathrm{d}(\Phi, \Psi ; x)<\varepsilon^{\prime}
$$

Since $\Re$ is nonatomic, if $z_{0} \neq \mathrm{B}(H)$ we can construct a countably infinite partition $\left\{y_{i}\right\}$ of $z_{0}^{\prime}$ with $\mathrm{d}\left(\Phi, \Psi ; y_{i}\right) \geq \varepsilon^{\prime}$. Writing $\mathrm{H}\left(z_{0}^{\prime}\right)$ as an ITPS $\otimes \mathrm{H}\left(y_{\imath}\right)$ we see that $(\Phi, \Psi)=0$ which is not possible by lemma 6.4. Hence $z_{0}=\mathrm{B}(H)$.

Let $x_{a}$ be a well ordering of all $x$ satisfying Eq. (6.20). Then $y_{a}=x_{a}-$ $\underset{\alpha^{\prime}<\alpha}{\vee} x_{\alpha^{\prime}}$ is a partition of $\mathrm{B}(H)$. Since $y<x$ implies $\mathrm{d}(\Phi, \Psi ; y) \leq \mathrm{d}(\Phi, \Psi ; x)$, each $y_{\infty}$ satisfies Eq. (6.20). Since

$$
\Pi_{\omega}\left[1-\mathrm{d}\left(\Phi, \Psi ; y_{\omega}\right)\right]=\operatorname{Tr}[\mathrm{P}(\Phi) \mathrm{P}(\Psi)]>0
$$

it follows that

$$
\sum_{\omega} \mathrm{d}\left(\Phi, \Psi ; y_{\omega}\right)<\infty
$$


Hence there exists a finite set $J$ of $y_{a}$ such that $y=\vee y_{\beta}, y_{\beta} \notin J$ satisfies $\mathrm{d}(\Phi, \Psi ; y)<\varepsilon^{\prime}$. Then $z_{\infty} \wedge y, z_{\infty} \wedge y_{\beta}, y_{\beta} \in J$ is a partition of each $z_{\infty}$. Let $B^{\prime}=\left\{z_{\alpha \beta}\right\}$ be the corresponding subpartition of $B$. By construction, Eq. (6.18) is satisfied.

If $\Pi_{j}\left(1-a_{j}\right)=A$ where $0 \leq a_{j}<1$, then the power series expansion of $\log (1-x)$ gives the inequality

$$
0<\sum a_{j}<-\log A
$$

Thus Eq. (6.16) implies that

$$
\sum \mathrm{d}\left(\Phi, \Psi ; z_{\alpha \beta}\right)<-\log \{\operatorname{Tr}[\mathrm{P}(\Phi) \mathrm{P}(\Psi)]\}
$$

Eq. (6.20) now implies

Q.E.D.

$$
\sum \mathrm{d}\left(\Phi, \Psi ; z_{\alpha \beta}\right)^{2}<\varepsilon
$$

Lemma 6.6: If $1+\delta=\Pi\left(1+\delta_{j}\right)$ where $\delta_{j}>0$ and $\delta<1$ then

$$
\left|\delta-\sum \delta_{j}\right|>\delta^{2}
$$

Proof: We have

$$
\begin{aligned}
1+\delta & =1+\sum_{j} \delta_{j}+\sum_{j_{1}<j_{2}} \delta_{j_{1}} \delta_{j_{2}}+\cdots \\
& <1+\sum \delta_{j}+\left(\sum \delta_{j}\right)^{2} / 2 !+\cdots
\end{aligned}
$$

Noting that $\sum \delta_{j}<\delta$, it follows from this equation that

$$
\delta-\sum \delta_{j}<\delta^{2} / 2 !+\delta^{3} / 3 !+\cdots
$$

If $\delta<1$ then $e^{\delta}-\delta-1<\delta^{2}$ and this gives the desired result. Q.E.D.

We now proceed to construct the linear space $K$ from the factorizable vectors. The following is motivated by Eqs. (5.17-20). Choose some fixed unit factorizable vector $\Omega$, and fix $\Omega(x),\|\Omega(x)\|=1$, for each $x \in \Re$. Then for any finite partition $B$ we have

$$
\Omega=\mathrm{C}(B) \otimes_{i} \Omega\left(z_{i}\right) \quad \text { where } \quad|\mathrm{C}(B)|=1
$$

Let $\Psi$ be any factorizable vector with $(\Omega, \Psi)=1$, and choose $\Psi(x)$ for each $x$ so that

$$
(\Omega(x), \Psi(x))=1
$$


Let

$$
\Psi^{\prime}(x)=\Psi(x)-\Omega(x)
$$

Then

$$
\left(\Psi^{\prime}(x), \Omega(x)\right)=0
$$

Let $K$ be the collection of all factorizable vectors $\Psi \in H$ with $(\Omega, \Psi)=1$. We shall denote the elements of $K$ by $\log \Psi$. The following lemma will allow us to define a linear structure in $K$.

Lemma 6. 7: Let $\log \Psi_{1}, \log \Psi_{2} \in K$ and let $C_{1}, C_{2}$ be complex numbers. For any finite partition $B=\left\{z_{i}\right\}$ define

$$
\Psi(B)=\mathrm{C}(B) \otimes_{i}\left\{\Omega\left(z_{i}\right)+C_{1} \Psi_{1}^{\prime}\left(z_{i}\right)+C_{2} \Psi_{2}^{\prime}\left(z_{i}\right)\right\}
$$

Then

$$
\Psi=\text { strong } \lim _{\beta \downarrow} \Psi(B) \equiv \bigcap_{B}\left\{\bigcup_{B^{\prime}<B} \Psi\left(B^{\prime}\right)\right\}^{(\text {s) }}
$$

(where $B, B^{\prime}$ are finite partitions) exists and is unique, and is a factorizable vector with $(\Omega, \Psi)=1$.

Proof: For any $B, \Psi(B)$ is a product vector with $(\Omega, \Psi(B))=1$. If $\Psi$ is any limit vector it obviously satisfies $(\Omega, \Psi)=1$, and it follows from lemma 3.3 that $\Psi$ is factorizable. To prove the existence and uniqueness, we show that for any $\varepsilon>0$ there exists some finite partition $B$ such that $B^{\prime}<B$ implies

$$
\left\|\Psi\left(B^{\prime}\right)-\Psi(B)\right\|^{2}<\varepsilon
$$

By lemma 6.5 we can choose a finite partition $B=\left\{z_{i}\right\}$ such that

$$
\left\|\Psi_{k}^{\prime}\left(z_{i}\right)\right\|^{2}<\varepsilon \quad \text { and } \quad \sum_{i}\left\|\Psi_{k}^{\prime}\left(z_{i}\right)\right\|^{4}<\varepsilon, \quad k=1,2
$$

Let $B^{\prime}=\left\{z_{i j}\right\}$ where $z_{i}=\bigvee_{j} z_{i j}$, be a subpartition of $B$. If $\varepsilon<1$, it follows from lemma 6.6 that

$$
\left|\left\|\Psi_{k}^{\prime}\left(z_{i}\right)\right\|^{2}-\sum_{j}\left\|\Psi_{k}^{\prime}\left(z_{i j}\right)\right\|^{2}\right|<\left\|\Psi_{k}^{\prime}\left(z_{i}\right)\right\|^{4}, \quad k=1,2
$$

Now

$$
\Psi_{k}^{\prime}\left(z_{i}\right)=\chi_{k i}+\Psi_{k i}, \quad k=1,2
$$

where $\left|A_{i}\right|=1$, and 


$$
\begin{aligned}
\Psi_{k i}= & A_{i}\left\{\sum_{j_{1}<j_{2}} \Psi_{k}^{\prime}\left(x_{i_{j_{1}}}\right) \otimes \Psi_{k}^{\prime}\left(x_{i_{j_{2}}}\right) \otimes\left[\bigotimes_{j_{\neq^{j} j^{\prime} j_{2}}} \Omega\left(z_{i j}\right)\right]+\cdots\right. \\
& +\cdots \\
\chi_{k i}= & A_{i} \sum_{j} \Psi_{k}^{\prime}\left(z_{i j}\right) \otimes\left[\bigotimes_{j^{\prime} \neq^{j}} \Omega\left(z_{i j^{\prime}}\right)\right]
\end{aligned}
$$

In evaluating $z_{i}$ component of $\Psi\left(B^{\prime}\right)$, we encounter an expression of the same form as the right hand sides of (6.35) and (6.36) where $\Psi_{k}^{\prime}(z)_{i j}$ is to be replaced by $C_{1} \Psi_{1}^{\prime}\left(z_{i j}\right)+C_{2} \Psi_{2}^{\prime}\left(z_{i j}\right)$. We write $\Psi_{k i}$ and $\chi_{k i}$ in this case as $\Psi_{3 i}$ and $\chi_{3 i}$. Then we can write

$$
\begin{aligned}
& \Psi(B)=C(B) \otimes_{i}\left(\Omega\left(z_{i}\right)+\chi_{3 i}+C_{1} \Psi_{1 i}+C_{2} \Psi_{2 i}\right), \\
& \Psi\left(B^{\prime}\right)=C(B) \otimes_{i}\left(\Omega\left(z_{i}\right)+\chi_{3 i}+\Psi_{3 i}\right)
\end{aligned}
$$

where first two terms in parentheses are orthogonal to the rest. If we write $\chi(B)=C(B) \otimes_{i}\left(\Omega\left(z_{i}\right)+\chi_{3 i}\right)$, then

$$
\|\Psi(B)-\chi(B)\|^{2} \leqq\|\chi(B)\|^{2}\left[\exp \sum_{i}\left\{\left\|C_{1} \Psi_{1 i}+C_{2} \Psi_{2 i}\right\|^{2} /\left(1+\left\|\chi_{3 i}\right\|^{2}\right)\right\}-1\right]
$$

and a similar evaluation for $\Psi\left(B^{\prime}\right)$. Obviously, we may omit $\left\|\chi_{3 i}\right\|^{2}$ on the right hand side of (6.39). From equations (6.36) and (6.34), we have

$$
\begin{aligned}
\left\|\Psi_{k i}\right\|^{2} & <\sum_{n=2}^{\infty}(n !)^{-1}\left(\sum_{j} \| \Psi_{k}^{\prime}\left(z_{i j}\right) i^{2}\right)^{n} \\
& <\sum_{n=2}^{\infty}(n !)^{-1}\left(\left\|\Psi_{k}^{\prime}\left(z_{i}\right)\right\|^{2}+\mid i \Psi_{k}^{\prime}\left(z_{i}\right) \|^{4}\right)^{n}<\left\|\Psi_{k}^{\prime}\left(z_{i}\right)\right\|^{4}
\end{aligned}
$$

for small $\varepsilon$. We then have

$$
\begin{aligned}
\sum \| C_{1} \Psi_{1 i}+C_{2} \Psi_{2 i}||^{2} & \leqq\left. 2\left|C_{1}\right|^{2} \sum_{i}|| \Psi_{1 i}\right|^{2}+2\left|C_{2}\right|^{2} \sum_{i}|| \Psi_{2 i} \|^{2} \\
& <2 \varepsilon\left\{\left|C_{1}\right|^{2}+\left|C_{2}\right|^{2}\right\}
\end{aligned}
$$

A similar estimate holds for $\sum_{i}|| \Psi_{3 i} \|^{2}$. Furthermore

$$
\begin{aligned}
\|\chi(B)\|^{2} & =\Pi_{i}\left(1+\sum_{j}|| C_{1} \Psi_{1}^{\prime}\left(z_{i j}\right)+C_{2} \Psi_{2}^{\prime}\left(z_{i j}\right) \|^{2}\right) \\
& \leqq \| \Psi_{1}||^{4\left|C_{1}\right|^{2}}|| \Psi_{2}||^{4\left|C_{2}\right|^{2}}
\end{aligned}
$$

is bounded uniformly in $B$.

Therefore

$$
\left\|\Psi\left(B^{\prime}\right)-\Psi(B)\right\|^{2}=0(\varepsilon)
$$

Q.E.D. 
Lemma 6. 8: Let $\log \Psi_{1}, \log \Psi_{2} \in K$, and let $\Psi$ be the unique limit vector of the preceding lemma. The equation

$$
\log \Psi=C_{1} \log \Psi_{1}+C_{2} \log \Psi_{2}
$$

defines a linear structure in the space $K$.

Proof: From the proof of the preceding lemma it is clear that ${ }^{38 a}$

$$
\lim _{B \downarrow} \sum_{i}\left\|\Psi^{\prime}\left(z_{i}\right)-C_{1} \Psi_{1}^{\prime}\left(z_{i}\right)-C_{2} \Psi_{2}^{\prime}\left(z_{i}\right)\right\|^{2}=0
$$

The linear structure follows from this easily. For example, if $\log \Psi_{a}=$ $C_{1}\left(C_{2} \log \Psi\right)$ and $\log \Psi_{b}=\left(C_{1} C_{2}\right) \log \Psi$, then $\sum_{i}\left\|\Psi_{a}^{\prime}\left(z_{i}\right)-\Psi_{b}^{\prime}\left(z_{i}\right)\right\|^{2} \rightarrow 0$ follows from (6.45) and inequality $\|\alpha+\beta\|^{2} \leqq 2\|\alpha\|^{2}+2\|\beta\|^{2}$. It then follows that $\Psi_{a}=\Psi_{b}$.

Lemma 6.9: The equation

$$
\left(\log \Psi_{1}, \log \Psi_{2}\right)=\lim _{B \uparrow}\left[\sum_{j}\left(\Psi_{1}^{\prime}\left(z_{j}\right), \Psi_{2}^{\prime}\left(z_{j}\right)\right)\right]
$$

(where $B=\left\{z_{j}\right\}$ is a finite partition and $\lim _{B \uparrow} \mathrm{f}(B)$ means $\bigcap_{B}\left\{\bigcup_{B^{\prime}<B} \mathrm{f}\left(B^{\prime}\right)\right\}^{-}$and \{\}$^{-}$is the closure of the set \{\}$)$defines an inner product on the space $K$ such that

$$
\left(\Psi_{1}, \Psi_{2}\right)=e^{\left(\log \Psi_{1}, \log \Psi_{2}\right)}
$$

$K$ is complete with respect to this inner product.

Proof: As in lemma 6.7 choose a finite partition $B=\left\{z_{j}\right\}$ so that

$$
\sum_{j}\left\{\left\|\Psi_{1}^{\prime}\left(z_{j}\right)\right\|^{4}+\left\|\Psi_{2}^{\prime}\left(z_{j}\right)\right\|^{4}\right\}<\varepsilon
$$

Then similar arguments give

$$
\begin{aligned}
\log \left(\Psi_{1}, \Psi_{2}\right) & =\log \left\{\Pi_{j}\left[1+\left(\Psi_{1}^{\prime}\left(z_{j}\right), \Psi_{2}^{\prime}\left(z_{j}\right)\right)\right]\right\} \\
& =\sum_{j}\left(\Psi_{1}^{\prime}\left(z_{j}\right), \Psi_{2}^{\prime}\left(z_{j}\right)\right)+0(\varepsilon)
\end{aligned}
$$

This proves the existence and uniqueness of $\left(\log \Psi_{1}, \log \Psi_{2}\right)$ and also proves Eq. (6.47). To prove linearity, we use similar arguments to get

38a) Use the inequality ||$\otimes\left(\Omega_{i}+\chi_{i}\right)-\left.\otimes\left(\Omega_{i}+\chi_{i}{ }^{\prime}\right) !\right|^{2} \geqq \sum_{i}|| \chi_{i}-\chi_{i}^{\prime}||^{2}$ if $\chi_{i}, \chi_{i}^{\prime} \perp \Omega_{i}$. 


$$
\begin{aligned}
(\log & \left.\Psi_{3}, C_{1} \log \Psi_{1}+C_{2} \log \Psi_{2}\right) \\
& =\sum_{j}\left(\Psi_{3}^{\prime}\left(z_{j}\right), C_{1} \Psi_{1}^{\prime}\left(z_{j}\right)+C_{2} \Psi_{2}^{\prime}\left(z_{j}\right)\right)+0(\varepsilon)^{1 / 2} \\
& =C_{1} \sum_{j}\left(\Psi_{3}^{\prime}\left(z_{j}\right), \Psi_{1}^{\prime}\left(z_{j}\right)\right)+C_{2} \Sigma_{j}\left(\Psi_{3}^{\prime}\left(z_{j}\right), \Psi_{2}^{\prime}\left(z_{j}\right)\right)+0(\varepsilon)^{1 / 2} \\
& =C_{1}\left(\log \Psi_{3}, \log \Psi_{1}\right)+C_{2}\left(\log \Psi_{3}, \log \Psi_{2}\right)+0(\varepsilon)^{1 / 2}
\end{aligned}
$$

To prove positive definiteness we note that by lemma 6.7 we can always write

$$
\sum_{i=1}^{n} C_{i} \log \Psi_{i}=\log \Psi .
$$

Thus it suffices to show that

$$
(\log \Psi, \log \Psi) \geq 0
$$

Now $(\Omega, \Psi)=1$ and $\|\Omega\|=1$ implies $\|\Psi\| \geq 1$. Using Eq. (6.47) we have

$$
(\log \Psi, \log \Psi)=\log (\Psi, \Psi) \geq 0
$$

Furthermore $\log (\Psi, \Psi)=0$ implies $\|\Psi\|=1$, hence $\Psi=\Omega$. Since $\log \Omega$ is clearly 0 in the linear structure given by Eq. (6.44), $\log (\Psi, \Psi)=0$ implies $\log \Psi=0$. This completes the proof of the positive definiteness.

It remains only to prove that $K$ is complete. Let $\log \Psi_{n}$ be a Cauchy sequence. Given $\varepsilon>0$, choose $N$ such that $n, m>N$ implies

$$
\left\|\log \Psi_{n}-\log \Psi_{m}\right\|^{2}<\varepsilon
$$

Hence $\left\|\Psi_{n}\right\|^{2}=\exp \left\|\log \Psi_{n}\right\|^{2}$ is bounded and

$$
\begin{aligned}
\left\|\Psi_{n}-\left(\Psi_{m}, \Psi_{n}\right) \Psi_{m} /\right\| \Psi_{m}\left\|^{2}\right\|^{2} & =\left(1-\exp -\left\|\log \Psi_{n}-\log \Psi_{m}\right\|^{2}\right) ! \mid \Psi_{n} \|^{2} \\
& <\left(1-e^{-\varepsilon}\right)_{1} \mid \Psi_{n} \|^{2}
\end{aligned}
$$

Due to $\left(\Omega, \Psi_{n}\right)=\left(\Omega, \Psi_{m}\right)=1$, we also have

$$
\begin{aligned}
\left|1-\left(\Psi_{m}, \Psi_{n}\right) /\right|\left|\Psi_{m}\right|^{2} \mid & =\left|\left(\Omega, \Psi_{n}-\left(\Psi_{m}, \Psi_{n}\right) \Psi_{m} /\left\|\Psi_{m}\right\|^{2}\right)\right| \\
& <\left(1-e^{-\varepsilon}\right)^{1 / 2}|| \Psi_{n}||
\end{aligned}
$$

Thus $\Psi_{n}$ is Cauchy. Clearly $\Psi=\lim \Psi_{n}$ satisfies $(\Omega, \Psi)=1$, and $\Psi$ is factorizable by lemma 3.3. By arguments similar to the above one can show that $\log \Psi=\lim \log \Psi_{n}$. Q.E.D.

It follows from the preceding lemmas that the mapping $\mathrm{e}^{\log \Psi} \rightarrow \Psi$ is one to one and isomorphic between total sets in $\mathrm{e}^{K}$ and $H$. Hence it can 
be extended by linearity and continuity to a unitary mapping of $\mathrm{e}^{K}$ onto $H$. We now construct the lattice $\mathfrak{P}=\log \mathfrak{R}$. For each $z \in \mathfrak{R}$, let

$$
\mathrm{P}(z)=\log z
$$

be the projection onto the subspace spanned by all $\log \Psi$ where $\Psi\left(z^{\prime}\right)=$ $\Omega\left(z^{\prime}\right)$. Clearly

$$
\log \left(z_{1} \wedge z_{2}\right)=\log z_{1} \wedge \log z_{2}
$$

and

$$
\log \left(z_{1} \bigvee z_{2}\right)=\log z_{1} \bigvee \log z_{2}
$$

and

$$
\log \left(z^{\prime}\right)=(\log z)^{\prime}
$$

This gives the desired nonatomic Boolean algebra of projections $\mathfrak{\beta}=\log \Re$. If $H$ is separable, then $K=\log H$ is separable and $\mathfrak{s}$ gives a direct integral decomposition of $K$. Then $\Re$ is a continuous tensor product factorization.

It remains to show that a different choice of $\Omega$ leads to a unitarily equivalent $(K, \mathfrak{P})$. Thus let $\Omega_{1}, \Omega_{2}$ be two unit factorizable vectors, and $\left(K_{1}, \mathfrak{P}_{1}\right),\left(K_{2}, \mathfrak{P}_{2}\right)$ the associated structures. For any factorizable $\Psi$, let $\Psi^{k}$ be the multiple of $\Psi$ satisfying $\left(\Psi^{k}, \Omega_{k}\right)=1, k=1,2$. In particular $\Omega_{1}^{1}=\Omega_{1}$ and $\Omega_{2}^{2}=\Omega_{2}$. By an elementary calculation we have

$$
\left(\log \Psi_{a}^{2}, \log \Psi_{b}^{2}\right)=\left(\log \Psi_{a}^{1}-\log \Omega_{2}^{1}, \log \Psi_{b}^{1}-\log \Omega_{2}^{1}\right)
$$

for any factorizable $\Psi_{a}, \Psi_{b} \in H$. Thus

$$
\log \Psi^{1}-\log \Omega_{2}^{1} \rightarrow \log \Psi^{2}
$$

gives a unitary mapping $U$ of $K_{1}$ onto $K_{2}$. By the definition of $\mathrm{P}(z)=$ $\log z$ we have

$$
\mathrm{P}_{k}(z) \log \Psi^{k}=\log \left[\Psi^{k}(z) \otimes \Omega_{k}\left(z^{\prime}\right)\right]
$$

where $\left(\Psi^{k}(z), \Omega_{k}(z)\right)=1, k=1,2$. Hence

$$
\begin{aligned}
\mathrm{P}_{1}(z)\left(\log \Psi^{1}-\log \Omega_{2}^{1}\right) & =\left\{\log \left[\Psi^{1}(z) \otimes \Omega_{1}\left(z^{\prime}\right)\right]-\log \Omega_{2}^{1}\right\} \\
& -\left\{\log \left[\Omega_{2}^{1}(z) \otimes \Omega_{1}\left(z^{\prime}\right)\right]-\log \Omega_{2}^{1}\right\}
\end{aligned}
$$

Under the unitary operator $U$ defined by Eq. (6.60) this vector is mapped into 


$$
\begin{aligned}
\log \left[\Psi^{2}(z) \otimes \Omega_{1}^{2}\left(z^{\prime}\right)\right]-\log \left[\Omega_{2}^{2}(z) \otimes \Omega_{1}^{2}\left(z^{\prime}\right)\right] \\
=\left[\log \Psi^{2}(z) \oplus \log \Omega_{1}^{2}\left(z^{\prime}\right)\right]-\left[0 \oplus \log \Omega_{1}^{2}\left(z^{\prime}\right)\right] \\
=\log \Psi^{2}(z) \oplus 0=\log \left[\Psi^{2}(z) \otimes \Omega_{2}\left(z^{\prime}\right)\right] \\
\quad=P_{2}(z) \log \Psi^{2}
\end{aligned}
$$

Thus $U P_{1} U^{-1}=P_{2}$, which proves the unitary equivalence of $\left(K_{1}, \mathfrak{P}_{1}\right)$ and $\left(K_{2}, \mathfrak{P}_{2}\right)$.

Finally, we note that $(K, \mathfrak{F})$ obtained from $H=\mathrm{e}^{K^{\prime}}, \mathfrak{R}=\mathrm{e}^{\mathfrak{B}^{\prime}}, \Omega=\mathrm{e}^{0}$, returns back to $\left(K^{\prime}, \mathfrak{\beta}^{\prime}\right)$. For, $\mathrm{e}^{\phi}, \phi \in K^{\prime}$ is a product vector and (5.3) and (6.47) shows that $K^{\prime} \subset K$. (5.12) then shows $K=K^{\prime} . \quad \mathfrak{B}=\mathfrak{P}^{\prime}$ follows from a similar arguments.

We summarize these results as follows

Theorem 6.1: Let $\mathfrak{R}$ be a complete nonatomic Boolean algebra of type $I$ factors on the Hilbert space $H$ such that $\inf _{B} \mathrm{~d}(\Psi ; B)>0$ for all $\Psi \in H$ where $B$ is a finite partition of $\mathrm{B}(H)$ in the lattice $\Re$. Then there is a Hilbert space $K$ and a nonatomic Boolean algebra of projections $\mathfrak{\beta}=\log \Re$ such that $(H, \Re)$ and $\left(\mathrm{e}^{K}, \mathrm{e}^{\Re}\right)$ are unitarily equivalent. Also $(H, \Re)$ determines $(K, \mathfrak{P})$ up to unitary equivalence. If $H$ is separable, then there is a direct integral decomposition

$$
K=\int^{\oplus} \mathrm{K}(z) \mathrm{d} \mu(z)
$$

so that $\Re$ is a continuous tensor product factoriaation. The unitary invariants of $\mathfrak{R}$ in the separable case are the equivalence class of the measure $\mu$ and the equivalence class of the dimension function dim $\mathrm{K}(z)$ which occur in the direct integral decomposition of $K$.

\section{Factorizable Vectors and Operators}

We now discuss factorizable vectors and operators for the structure $\left(\mathrm{e}^{K}, \mathrm{e}^{\mathfrak{B}}\right)$ where $\mathfrak{P}$ is a complete nonatomic Boolean algebra of projections. By the preceding construction, any factorizable vector in $H$ is of the form $C \mathrm{e}^{\phi}, \phi \in K$. Since $(K, \mathfrak{P})$ is determined up to unitary equivalence by $\left(\mathrm{e}^{K}, \mathrm{e}^{\mathfrak{B}}\right)$ it follows that the only factorizable vectors are the product vectors 
$C \mathrm{e}^{\phi}, \phi \in K$. We now prove that any factorizable operator $T \in \mathrm{B}\left(\mathrm{e}^{K}\right)$ must be of the form $C \mathrm{~T}(\psi, Q, \chi)$ (see Theorem 5.3).

Given $(K, \mathfrak{B})$ let $T \in \mathrm{B}\left(\mathrm{e}^{K}\right)$ be factorizable. Since $T \Omega$ is a factorizable vector, it follows from lemma 6.4 that $(\Omega, T \Omega) \neq 0$. Hence we can assume that $(\Omega, T \Omega)=1$. Now on product vectors $\mathrm{e}^{\phi}$ we have

$$
\mathrm{T}(\psi, Q, \chi) \mathrm{e}^{\phi}=e^{(x, \phi)} \mathrm{e}^{Q \phi+\psi}
$$

Except for the linearity of $Q$, it is trivial to show that $T$ must be of this form. Namely, since $(\Omega, T \Omega)=1$ we can define $\psi, \chi \in K$ by

$$
\begin{aligned}
& \mathrm{e}^{\psi}=T \Omega \\
& \mathrm{e}^{x}=T^{*} \Omega
\end{aligned}
$$

Then

$$
T^{\prime}=\mathrm{T}^{*}(-\psi) T \mathrm{~T}(-\chi)
$$

(see Eqs. (5.48-49)) is a factorizable operator satisfying

$$
\begin{aligned}
& T^{\prime} \Omega=\Omega \\
& \left(T^{\prime}\right)^{*} \Omega=\Omega
\end{aligned}
$$

It follows that $T^{\prime} \mathrm{e}^{\phi}$ is of the form $\mathrm{e}^{\mathrm{T}^{\prime}(\phi)}$. It remains only to prove that

$$
Q \phi=\mathrm{T}^{\prime}(\phi)
$$

defines a linear operator $Q$.

To prove the linearity of $Q$ we rederive Eq. (6.70) using the notation introduced in Eq. (6.26) et seq. Thus we write

$$
\begin{aligned}
& T \Omega=\mathrm{e}^{\log \hat{\psi}} \\
& T^{*} \Omega=\mathrm{e}^{\log \hat{x}}
\end{aligned}
$$

If $B=\left\{z_{i}\right\}$ is any partition of $\mathrm{B}(H)$ we have $T=\otimes \mathrm{T}\left(z_{i}\right)$. For each $x \in \mathfrak{P}$ choose $\mathrm{T}(x)$ so that

$$
(\Omega(x), \mathrm{T}(x) \Omega(x))=1
$$

Then

$$
\begin{aligned}
& \mathrm{T}(x) \Omega(x)=\Omega(x)+\hat{\psi}^{\prime}(x) \\
& \mathrm{T}^{*}(x) \Omega(x)=\Omega(x)+\hat{\chi}^{\prime}(x)
\end{aligned}
$$


Let $\mathrm{P}(x)$ be the projection onto $\Omega(x)$ and let

$$
\mathrm{Q}(x)=[1-\mathrm{P}(x)] \mathrm{T}(x)
$$

Then for any $\log \Phi \in K$ we have

$$
\mathrm{T}(x) \Phi^{\prime}(x)=\left(\hat{\chi}^{\prime}(x), \Phi^{\prime}(x)\right) \Omega(x)+\mathrm{Q}(x) \Phi^{\prime}(x)
$$

For any partition $B$ we have

$$
T \mathrm{e}^{\log \phi}=\mathrm{C}(B) \otimes_{i}\left[\mathrm{~T}\left(z_{i}\right) \Omega\left(z_{i}\right)+\mathrm{T}\left(z_{i}\right) \Phi^{\prime}\left(z_{i}\right)\right]
$$

Using Eqs. (6.74), (6.75), (6.77) and

$$
\Pi_{i}\left[1+\left(\hat{\chi}^{\prime}\left(z_{i}\right), \Phi^{\prime}\left(z_{i}\right)\right)\right]=e^{(\log \hat{x}, \log \Phi)}
$$

we have

$$
\begin{aligned}
T \mathrm{e}^{\log \Phi}= & e^{(\log \hat{x}, \log \Phi)} \mathrm{C}(B) \otimes\left\{\Omega\left(z_{i}\right)\right. \\
& \left.+\left[\hat{\psi}^{\prime}\left(z_{i}\right)+\mathrm{Q}\left(z_{i}\right) \Phi^{\prime}\left(z_{i}\right)\right] /\left[1+\left(\hat{\chi}^{\prime}\left(z_{i}\right), \Phi^{\prime}\left(z_{i}\right)\right)\right]\right\}
\end{aligned}
$$

Given any $\log \Phi \in K, \varepsilon>0$ it follows from lemma 6.5 that one can choose a finite partition $B$ so that

$$
\left|\left(\hat{\chi}^{\prime}\left(z_{i}\right), \Phi^{\prime}\left(z_{i}\right)\right)\right|<\varepsilon
$$

It follows that

$$
\lim _{B \downarrow} \sum_{i}\left\|\Psi^{\prime}\left(z_{i}\right)-\hat{\psi}^{\prime}\left(z_{i}\right)-\mathrm{Q}\left(z_{i}\right) \Phi^{\prime}\left(z_{i}\right)\right\|^{2}=0
$$

for $\Psi=(T \Phi) \exp -(\log \hat{\chi}, \log \Phi)$. We now have

$$
T \mathrm{e}^{\log \Phi}=e^{(\log \hat{\psi}, \log \Phi)} \mathrm{e}^{\log \hat{\psi}+Q \log \Phi}
$$

where $Q \log \Phi=\log \Psi-\log \hat{\psi}$ and

$$
\lim _{B \downarrow} \sum_{i}\left\|\left(\mathrm{e}^{Q \log \Phi}\right)^{\prime}\left(z_{i}\right)-\mathrm{Q}\left(z_{i}\right) \Phi^{\prime}\left(z_{i}\right)\right\|^{2}=0 .
$$

By lemma 6.7 we have

$$
\mathrm{e}^{C_{1} \log \Phi_{1}+C_{2} \log \Phi_{2}}=\lim _{B \downarrow} \mathrm{C}(B) \otimes\left\{\Omega\left(z_{i}\right)+C_{1} \Phi_{1}^{\prime}\left(z_{i}\right)+C_{2} \Phi_{2}^{\prime}\left(z_{i}\right)\right\}
$$

Using the continuity of $T$ we find

$$
\begin{aligned}
& T \mathrm{e}^{C_{1} \log \Phi_{1}+C_{2} \log \Phi_{2}}=e^{\left(\log \hat{x}, C_{1} \log \Phi_{1}+C_{2} \log \Phi_{2}\right)} \\
& \lim _{B \downarrow} \mathrm{C}(B) \otimes\left\{\Omega\left(z_{i}\right)+\hat{\psi}^{\prime}\left(z_{i}\right)+C_{1} \mathrm{Q}\left(z_{i}\right) \Phi_{1}^{\prime}\left(z_{i}\right)+C_{2} \mathrm{Q}\left(z_{i}\right) \Phi_{2}^{\prime}\left(z_{i}\right)\right\} \\
& =e^{\left(\log \hat{x}, C_{1} \log \Phi_{1}+C_{2} \log \Phi_{2}\right)} \mathrm{e}^{\log \hat{\psi}+C_{1} Q \log \Phi_{1}+C_{2} Q \log \Phi_{2}}
\end{aligned}
$$


which proves the linearity of $Q$. It is clear from Eq. (6.84) that $Q \in \mathfrak{A}^{\prime}$ where $\mathfrak{A}=\{P \in \mathfrak{F}\}\}^{\prime \prime}$. We summarize these results as Theorem 6.2.

Theorem 6.2: Let $K$ be a Hilbert space, and let $\mathfrak{P}$ be a complete nonatomic Boolean algebra of projections on $K . A$ vector $\Psi \in \mathrm{e}^{K}$ is factorizable if and only if it is a product vector $C \mathrm{e}^{\phi}, \phi \in K . \quad T \in \mathrm{B}\left(\mathrm{e}^{K}\right)$ is factorizable if and only if it is of the form $C \mathrm{~T}(\psi, Q, \chi)$ where $C$ is a complex number and $\mathrm{T}(\psi, Q, \chi)$ is given by Theorem 5.3.

\section{Application to the Group Integral for Irreducible Representation of the CCRs}

In this section we give an application to the group integral for irreducible representations of the CCRs introduced by Klauder and McKenna. ${ }^{17), 187}$

We first give a brief definition of a representation of the CCRs over an inner product space $V$. Given a representation of the CCRs over $V$, we associate a von Neumann algebra $\mathrm{R}(W)$ with every linear subset $W$ of $V$. We discuss the properties of the map $W \rightarrow \mathrm{R}(W)$. With any orthogonal basis $\left\{h_{\omega}\right\}_{\alpha \in A}$ of $V$, we associate a type I factorization $\left\{R_{\alpha}\right\}_{\alpha \in A}$ of $\mathrm{R}\left(V_{0}\right)$ where $V_{0}$ is the dense linear subset of all finite linear combinations of the $h_{a}$. We define the group integral and discuss its properties for finitedimensional $V$. We then consider the group integral for irreducible representztions of the CCRs over countably infinite dimensional $V$. We establish necessary and sufficient conditions that the group integral exists and has the desired value (Theorem 7.1). We prove that the group integral is independent of the order of integration if and only if the representation is a tensor product representation (Theorem 7.2).

\section{Representations of the CCRs}

For the sake of completeness, we give the definition of a representation of the CCRs. ${ }^{39}$ Let $V$ be a real inner product space. A representation

39) For a detailed and rigorous exposition of the representations of the CCRs see L. Gårding and A.S. Wightman, Proc. Nat. Acad. Sci. 40, 617 (1954); I.E. Segal, Trans. Am. Math. Soc. 88, 12 (1958); J. Lew, thesis, Princeton University (1960); H. Araki, thesis, Princeton University (1960); H. Araki, J. Math. Phys. 1, 492 (1960). 
of the CCRs over $V$ is the structure consisting of a complex Hilbert space $H$, and a map $f \times g \rightarrow \mathrm{U}(f, g)$ from $V \times V$ into the set of unitary operators on $H$ such that

$$
\mathrm{U}\left(f_{1}, g_{1}\right) \mathrm{U}\left(f_{2}, g_{2}\right)=\mathrm{U}\left(f_{1}+f_{2}, g_{1}+g_{2}\right) e^{i\left(f_{2}, g_{1}\right)}
$$

where $\left(f_{2}, g_{1}\right)$ is the real inner product of $f_{2}$ and $g_{1}$ in $V$, and such that for each $f, g$ the operator $\mathrm{U}(s f, t g)$ is weakly continuous in the real parameters $s, t$.

For finite-dimensional $V$, von Neumann proved that there exists a unique irreducible representation of the CCRs (usually called the Schrödinger representation), and that any representation is (unitarily equivalent to) a discrete direct sum of copies of the Schrödinger representation. ${ }^{40)}$ For infinite-dimensional $V$ there are many inequivalent irreducible representations of the CCRs over $V^{39}$ A classification of all irreducible tensor product representations of the CCRs has been given by Klauder, McKenna and Woods. ${ }^{18)}$

\section{A Lattice of von Neumann Algebras}

Let $f \times g \rightarrow \mathrm{U}(f, g)$ be a representation of the CCRs over $V$. For any linear subset $W$ of $V$ we define the von Neumann algebra

$$
\mathrm{R}(W)=\{\mathrm{U}(f, g) ; f, g \in W\}^{\prime \prime}
$$

We now discuss the properties of the map $W \rightarrow \mathrm{R}(W)$ where $W$ is a subspace (closed linear subset). The set of all subspaces $W$ of $V$ has a natural lattice structure given by

$$
\begin{aligned}
& W_{1} \wedge W_{2}=W_{1} \cap W_{2} \\
& W_{1} \vee W_{2}=\left(W_{1} \cup W_{2}\right)^{\perp \perp}
\end{aligned}
$$

where, $\cap, U$ denotes set theoretical intersection and union respectively, and

$$
W^{\perp}=\{x \in V:(x, y)=0 \text { for all } y \in W\}
$$

The map $W \rightarrow \mathrm{R}(W)$ is a lattice homomorphism if

40) J. von Neumann, Math. Ann. 104, 570 (1931). 


$$
\begin{aligned}
& \mathrm{R}\left(W_{1}\right) \wedge \mathrm{R}\left(W_{2}\right)=\mathrm{R}\left(W_{1} \wedge W_{2}\right) \\
& \mathrm{R}\left(W_{1}\right) \vee \mathrm{R}\left(W_{2}\right)=\mathrm{R}\left(W_{1} \vee W_{2}\right)
\end{aligned}
$$

The equation (7. 7) follows from the definition if $W_{1} \perp W_{2}$. It also follows from Eq. (7. 1) that

$$
\mathrm{R}(W)^{\prime} \supset \mathrm{R}\left(W^{\perp}\right)
$$

For irreducible representations, $\mathrm{R}(V)=\mathrm{B}(H)$ and Eq. (7. 7) gives

$$
\mathrm{R}(W) \vee \mathrm{R}\left(W^{\perp}\right)=\mathrm{B}(H)
$$

hence $\mathrm{R}(W)$ is a factor. Since the restriction of the map $f \times g \rightarrow \mathrm{U}(f, g)$ to $f, g \in W$ gives a representation of the CCRs over $W$, it follows from von Neumann's results ${ }^{40)}$ that $\mathrm{R}(W)$ is a type I factor for any finite-dimensional $W$.

For the Fock representation, Araki has shown that the map $W \rightarrow \mathrm{R}_{\mathrm{F}}(W)$ gives an isomorphism of these complemented lattices. ${ }^{4)}$ In addition it was shown that ${ }^{41)}$

$$
\mathrm{R}_{\mathrm{F}}(W)=\mathrm{R}_{\mathrm{F}}(\bar{W})
$$

for all linear subsets $W\left(\bar{W}=W^{\perp \perp}\right.$ is the subspace which is the closure of $W)$. This follows directly from the fact that $\mathrm{U}_{\mathrm{F}}(f, g)$ is strongly continuous in $f$ and $g$ with the strong topology of $V$ for $f$ and $g .{ }^{42}$ In general $\mathrm{U}(f, g)$ is not strongly continuous in the strong topology on $V$. Clearly

$$
\mathrm{R}(W) \subset \mathrm{R}(\bar{W})
$$

However there are examples where the equality does not hold. It is also possible that $\mathrm{R}(V)=\mathrm{B}(H)$ and $\mathrm{R}\left(V_{0}\right) \neq \mathrm{B}(H)$ for a dense set $V_{0}$ in $V$.

Given a representation of the CCRs over $V$, let $\left\{h_{\alpha}\right\}_{a \in A}$ be an orthogonal basis for $V$, and let

$$
\mathrm{R}_{\omega}=\mathrm{R}\left(W_{\omega}\right)
$$

where $W_{a}$ is the one-dimensional space spanned by $h_{a}$. Then $R_{a}$ is a type I factor and

41) H. Araki (reference 4), Eq. (3.5).

42) H. Araki and E.J. Woods (reference 12), lemma 2. 3. 


$$
\begin{aligned}
& R_{\infty} \subset R_{\beta}^{\prime}=1, \quad \alpha \neq \beta \\
& \vee_{\infty} R_{\infty}=\mathrm{R}\left(V_{0}\right)
\end{aligned}
$$

where $V_{0}$ is the dense linear subset of all finite linear combinations of the $h_{\alpha}$. Thus $R_{\alpha}$ is a type I factorization of $\mathrm{R}\left(V_{0}\right)$ (see definition 1.1).

\section{The Group Integral: Finite Dimensional Case}

We now give the definition and basic properties of the group integral introduced by Klauder and McKenna. ${ }^{17), 18)}$ We consider first the case where $V$ is finite dimensional. Let $\left\{h_{n}\right\}_{n \in I_{N}}$ be a basis for $V$. Then for any $f, g \in V$ we can write $f=\sum p_{n} h_{n}, g=\sum q_{n} h_{n}$. For any $\Phi_{1}, \Phi_{2}, \Psi_{, 1}$ $\Psi_{2} \in H$ we define the group integral

$$
\mathrm{I}\left(\Phi_{1}, \Psi_{1} ; \Phi_{2}, \Psi_{2}\right)=\int \mathrm{d} \mu(f, g)\left(\mathrm{U}(f, g) \Phi_{1}, \Psi_{1}\right) *\left(\mathrm{U}(f, g) \Phi_{2}, \Psi_{2}\right)
$$

where

$$
\mathrm{d} \mu(f, g)=(2 \pi)^{-N} \prod_{n=1}^{N} \mathrm{~d} p_{n} \mathrm{~d} q_{n}
$$

and the domain of integration is $R_{N} \times R_{N}$. For the special case $\Phi_{1}=\Phi_{2}=$ $\Psi_{1}=\Psi_{2}=\Psi$, we just write

$$
\mathrm{I}(\Psi)=\mathrm{I}(\Psi, \Psi ; \Psi, \Psi)
$$

For irreducible representations of the CCRs over a finite dimensional $V$, it has been shown that ${ }^{43}$

$$
\mathrm{I}\left(\Phi_{1}, \Psi_{1} ; \Phi_{2}, \Psi_{2}\right)=\left(\Psi_{1}, \Psi_{2}\right)\left(\Phi_{2}, \Phi_{1}\right)
$$

We now discuss the group integral for a reducible representation of the CCRs over a finite dimensional $V$. By von Neumann's results we can write

$$
H=H^{\prime} \otimes H^{\prime \prime}
$$

and

$$
\mathrm{U}(f, g)=\mathrm{U}^{\prime}(f, g) \otimes \mathbf{1}
$$

where the $\mathrm{U}^{\prime}(f, g)$ are an irreducible representation of the CCRs on $H^{\prime}$.

43) J.R. Klauder, J. McKenna, and E.J. Woods (reference 18), theorem 4.1. 
Let $\alpha_{i}, \beta_{j}$ be orthonormal bases for $H^{\prime}, H^{\prime \prime}$ respectively. Given $\Psi_{1}$, $\Psi_{2}, \Phi_{1}, \Phi_{2} \in H$, let $A, B, C, D$ respectively be their coefficient matrices. That is

etc. Then

$$
\Psi_{1}=\sum A_{i j} \alpha_{i} \otimes \beta_{j}
$$

$$
\left\|\Psi_{1}\right\|^{2}=\operatorname{Trace} A^{*} A
$$

etc. and

$$
\begin{aligned}
& \mathrm{I}\left(\Phi_{1}, \Psi_{1} ; \Phi_{2}, \Psi_{2}\right)=\sum \bar{A}_{i j} C_{k l} \bar{D}_{m m} B_{0 p} \\
& \quad \int \mathrm{d} \mu(f, g)\left(\mathrm{U}^{\prime}(f, g) \alpha_{k}, \alpha_{i}\right)^{*}\left(\mathrm{U}^{\prime}(f, g) \alpha_{m}, \alpha_{0}\right)\left(\beta_{l}, \beta_{j}\right)^{*}\left(\beta_{n}, \beta_{p}\right)
\end{aligned}
$$

Using Eq. (7.18) for the irreducible $U^{\prime}$ we get

$$
\mathrm{I}\left(\Phi_{1}, \Psi_{1} ; \Phi_{2}, \Psi_{2}\right)=\text { Trace } A^{*} B D^{*} C
$$

Now

$$
\begin{aligned}
\left|\operatorname{Tr} A^{*} B D^{*} C\right| & \leq\left\{\operatorname{Tr}\left(A^{*} B\right)\left(A^{*} B\right)^{*}\right)^{1 / 2}\left\{\operatorname{Tr}\left(D^{*} C\right)\left(D^{*} C\right)^{*}\right\}^{1 / 2} \\
& \leq\left\{\operatorname{Tr}\left(A^{*} A\right)^{2} \operatorname{Tr}\left(B^{*} B\right)^{2} \operatorname{Tr}\left(C^{*} C\right)^{2} \operatorname{Tr}\left(D^{*} D\right)^{2}\right\}^{1 / 4}
\end{aligned}
$$

Since

$$
\operatorname{Tr}\left(A^{*} A\right)^{2} \leq\left(\operatorname{Tr} A^{*} A\right)^{2}=\left\|\Psi_{1}\right\|^{4}
$$

etc. we get

$$
\left|\mathrm{I}\left(\Phi_{1}, \Psi_{1} ; \Phi_{2}, \Psi_{2}\right)\right| \leq\left\|\Psi_{1}|||| \Psi_{2}||\right\| \Phi_{2}||\left\|\Phi_{1}\right\|
$$

Lemma 7.1: If $\|\Phi-\Psi\|<\varepsilon$, then

$$
|\mathrm{I}(\Phi)-\mathrm{I}(\Psi)|<(|| \Phi \|+\varepsilon)^{4}-\|\Phi\|^{4}
$$

Proof: Write $\Psi=\Phi+\chi$, and note that $\mathrm{I}(\Psi, \Psi ; \Psi, \Psi)$ is a linear function of each of its four arguments. It follows that $I(\Psi)-I(\Phi)$ is a sum of 15 terms of the form $\mathrm{I}(\chi, \Phi ; \Phi, \Phi), \mathrm{I}(\chi, \chi ; \Phi, \Phi)$ etc. The desired result then follows from Eq. (7.27). Q.E.D.

The property which makes the group integral interesting is Eq. (7.18). Thus the desired value for $\mathrm{I}\left(\Phi_{1}, \Psi_{1} ; \Phi_{2}, \Psi_{2}\right)$ is

$$
\left(\Psi_{1}, \Psi_{2}\right)\left(\Phi_{2}, \Phi_{1}\right)=\left(\operatorname{Trace} A^{*} B\right)\left(\operatorname{Trace} C D^{*}\right)
$$

But in general

$$
\text { Trace } A^{*} B D^{*} C \neq\left(\operatorname{Trace} A^{*} B\right)\left(\operatorname{Trace} C D^{*}\right)
$$


In particular, if we set $A=B=C=D$, then we have

$$
\text { Trace }\left(A^{*} A\right)^{2} \leq\left(\text { Trace } A^{*} A\right)^{2}
$$

with equality holding if and only if $A^{*} A$ is a one-dimensional projection operator, or equivalently, if the vector $\Psi=\sum A_{i j} \alpha_{i} \otimes \beta_{j}$ is a product vector (cf. Eqs. (2.12)-(2.14). For these reasons, the group integral for reducible representations is not so interesting. However it is used to defined the group integral for irreducible representations of the CCRs over an infinite-dimensioanl $V$, and we shall have odcasion to make use of the above properties.

The Group Integral: Infinite Dimensional Case

We consider a countably infinite dimensional $V$ with an orthogonal basis $\left\{h_{i}\right\}_{i \in I_{\infty}}$. Given a representation of the CCRs over $V$ on a complex Hilbert space $H$, let

$$
R_{i}=\left\{\mathrm{U}\left(s h_{i}, t h_{i}\right) ;-\infty<s, t<\infty\right\}^{\prime \prime}
$$

In the remainder of this section we consider representations such that $\vee_{i} R_{i}=\mathrm{B}(H)$. Then $\left\{R_{i}\right\}_{i \subseteq I_{\infty}}$ is a type $\mathrm{I}$ factorization (see Eq. (7.12) et seq.).

We now define the group integral $\mathrm{I}\left(\Phi_{1}, \Psi_{1} ; \Phi_{2}, \Psi_{2}\right)$. For each $N \in I_{\infty}$ we have a reducible representation of the CCRs over the finite-dimensional subspace of $V$ spanned by $\left\{h_{i}\right\}_{i \in I_{N}}$. For any $\Phi_{1}, \Psi_{1}, \Phi_{2}, \Psi_{2} \in H$, let $\mathrm{I}_{N}\left(\Phi_{1}, \Psi_{1} ; \Phi_{2}, \Psi_{2}\right)$ be the group integral defined by Eq. (7.15) for this reducible representation. Then we define

$$
\mathrm{I}\left(\Phi_{1}, \Psi_{1} ; \Phi_{2}, \Psi_{2}\right)=\lim _{N \rightarrow \infty} \mathrm{I}_{N}\left(\Phi_{1}, \Psi_{1} ; \Phi_{2}, \Psi_{2}\right)
$$

It has been shown that for irreducible tensor product representations, that is representations where $H$ is an ITPS $H=\bigotimes_{i=1}^{\infty} H_{i}$ and

$$
R_{i}=\mathrm{B}\left(H_{i}\right) \underset{j \neq i}{\otimes}\left\{\otimes \mathbb{1}_{j}\right\}
$$

that $^{18)}$

$$
\lim _{N \rightarrow \infty} I_{N}\left(\Phi_{1}, \Psi_{1} ; \Phi_{2}, \Psi_{2}\right)=\left(\Psi_{1}, \Psi_{2}\right)\left(\Phi_{2}, \Phi_{1}\right)
$$


We now establish the conditions under which the group integral exists and has the desired value.

Assume that for some unit vector $\Psi \in H$, we have

$$
\lim _{N \rightarrow \infty} \mathrm{I}_{N}(\Psi)=\|\Psi\|^{4}=1
$$

For each $N$ we can write

where

$$
H=H_{N} \otimes H_{N}^{\prime}
$$

$$
\mathrm{R}\left(I_{N}\right)=\bigwedge_{i=1}^{N} R_{i}
$$

is irreducible on $H_{N}$. Let $A_{N}$ be the coefficient matrix for $\Psi$ in some expansion

$$
\Psi=\sum A_{N i j} \alpha_{N i} \otimes \beta_{N j} ; \alpha_{N i} \in H_{N}, \beta_{N j} \in H_{N}^{\prime}
$$

Then Eq. (7.24) gives

$$
\mathrm{I}_{N}(\Psi)=\operatorname{Trace}\left(A_{N}^{*} A_{N}\right)^{2}
$$

Thus Eq. (7.35) implies that

$$
\lim _{N \rightarrow \infty} \operatorname{Trace}\left(A_{N}^{*} A_{N}\right)^{2}=1
$$

Since $\operatorname{Tr} A_{N}^{*} A=\|\Psi\|^{2}=1$, lemma 2.2 gives

$$
\mathrm{d}\left(\Psi ; \mathrm{R}\left(I_{N}\right), \mathrm{R}\left(I_{N}^{\mathrm{c}}\right)\right) \geq \operatorname{Trace}\left(A_{N}^{*} A_{N}\right)^{2}
$$

It follows that

$$
\lim _{N \rightarrow \infty} \mathrm{d}\left(\Psi ; \mathrm{R}\left(I_{N}\right), \mathrm{R}\left(I_{N}^{\mathrm{c}}\right)\right)=1
$$

By lemma 4. 10 this is a sufficient (but not necessary) condition that $R_{n}$ is a partial tensor product factorization.

We now note that Eq. (7.42) implies Eq. (7.34) for all $\Phi_{1}, \Psi_{1}, \Phi_{2}$, $\Psi_{2} \in H$. By lemma 4.10 there exist minimal projedtions $P_{N} \in \mathrm{R}\left(\mathrm{I}_{N}^{\mathrm{c}}\right)$ such that $P_{N} \rightarrow 1$ strongly as $N \rightarrow \infty$. The proof of Eq. (7.34) given by Klauder, McKenna and Woods ${ }^{18)}$ was based on the existence of such projections. We summarize these results as 
Theorem 7. 1: Let $V$ be a countably infinite dimensional inner product space with an orthogonal basis $\left\{h_{i}\right\}_{i \in I_{\infty}{ }^{*}}$ Let $U(f, g)$ be a representation of the CCRs over $V$ such that $\vee_{i} R_{i}=B(H)$ where

$$
R_{i}=\left\{\mathrm{U}\left(s h^{2}, t h_{i}\right) ;-\infty<s, t<\infty\right\}^{\prime \prime}
$$

The following conditions are equivalent.

(1) For some $\Psi \in H, \lim _{N \rightarrow \infty} I_{N}(\Psi)=\|\Psi\|^{4}$

(2) For all $\Phi_{1}, \Phi_{2}, \Psi_{1}, \Psi_{2} \in H$,

$$
\lim _{N \rightarrow \infty} I_{N}\left(\Phi_{1}, \Psi_{1} ; \Phi_{2}, \Psi_{2}\right)=\left(\Psi_{1}, \Psi_{2}\right)\left(\Phi_{2}, \Phi_{1}\right)
$$

(3) There exists a sequence of minimal projections $P_{N} \in \mathrm{R}\left(I_{N}^{\mathrm{c}}\right)$ such that $P_{N} \rightarrow 1$ strongly as $N \rightarrow \infty$ (which implies that $R_{i}$ is a partial tensor product factorization).

We now show that if Eq. (7.34) holds independent of the order of integration for some vectors then all $\mathrm{R}(J)=\bigvee_{i \in j} R_{i}$ are type I. It then follows from Theorem 4. 1 that the representation of the CCRs is a tensor product representation. By Theorem 7.1 and lemma 4.10 we can write $H$ as a partial tensor product

$$
H=\bigotimes_{k=1}^{\infty} \mathrm{H}\left(J_{k}\right)
$$

where

$$
\mathrm{H}\left(J_{k}\right)=\bigotimes_{n \in T_{k}} H_{n}
$$

and for $n \in J_{k}$ we have

$$
R_{n}=\left\{\mathrm{B}\left(H_{n}\right) \otimes\left[\underset{\substack{m \in I_{k} \\ m \neq n}}{\otimes} \mathbb{1}_{m}\right]\right\} \otimes\left\{\underset{k^{\prime} \neq k}{\otimes} \mathbb{1}_{k^{\prime}}\right\}
$$

For any $J \subset I_{\infty}$ let $J_{k}^{\prime}=J \cap J_{k}, J_{k}^{\prime \prime}=J^{\mathrm{c}} \cap J_{k}$. Then $J=\cup_{k} J_{k}^{\prime}$. We assume $\mathrm{R}(J)$ is not type $\mathrm{I}$ and get a contradiction. Let $\Phi=\otimes \Phi_{k}$, $\left\|\Phi_{k}\right\|=1$, be some unit product vector. If $\mathrm{R}(J)$ is not type $\mathrm{I}$, then ${ }^{29}$

$$
\sum_{k}\left\{1-\mathrm{d}\left(\Phi_{k} ; \mathrm{R}\left(J_{k}^{\prime}\right), \mathrm{R}\left(J_{k}^{\prime \prime}\right)\right\}=\infty\right.
$$

which implies that ${ }^{23)}$

$$
\prod_{k=1}^{\infty} d\left(\Phi_{k} ; \mathrm{R}\left(J_{k}^{\prime}\right), \mathrm{R}\left(J_{k}^{\prime \prime}\right)\right)=0
$$


Consider the irreducible representation of the CCRs over all $h_{n}, n \in J_{k}$ defined on $\mathrm{H}\left(J_{k}\right)$. Let $I_{k}\left(\Phi_{k}\right), \mathrm{I}_{k}^{\prime}\left(\Phi_{k}\right)$ denote the group integral taken over all $h_{n}, n \in J_{k}, J_{k}^{\prime}$ respectively. We have

$$
\mathrm{I}_{k}\left(\Phi_{k}\right)=1
$$

and from Eqs. (7.24) and (7.41) we have

$$
\mathrm{I}_{k}^{\prime}\left(\Phi_{k}\right) \leq \mathrm{d}\left(\Phi_{k} ; \mathrm{R}\left(J_{k}^{\prime}\right), R\left(J_{k}^{\prime \prime}\right)\right)
$$

Thus Eq. (7.47) implies that

$$
\prod_{k=1}^{\infty} I_{k}^{\prime}\left(\Phi_{k}\right)=0
$$

We now reorder the $h_{n}$ so that the integral over the first $N$ degrees of freedom $\rightarrow 0$ as $N \rightarrow \infty$. Choose $j_{m}$ so that

$$
\prod_{k=m}^{j_{m}} \mathrm{I}_{k}^{\prime}\left(\Phi_{k}\right)<2^{-m}
$$

and order the degrees of freedom according to the arrangement $J_{1}^{\prime}, J_{2}^{\prime}, \cdots$, $J_{j_{1}}^{\prime}, J_{1}^{\prime \prime}, J_{j_{1}+1}^{\prime}, \cdots, J_{j_{2}}^{\prime}, J_{2}^{\prime \prime}, J_{j_{3}+1}^{\prime}, \cdots$ etc. Now consider the group integral taken over the $N$ degrees of freedom up to and including $J_{j_{m}}^{\prime}$. Since the measure $\mathrm{d}(f, g)$ is a product measure, and $\Phi$ is a product vector, it follows from Eq. (7.15) that

$$
\mathrm{I}_{N}(\Phi)=\Pi \mathrm{I}_{k}^{\prime}\left(\Phi_{k=m}^{j_{m}}\right)<2^{-m}
$$

which implies $\lim I_{N}(\Phi)=0$. We now show that for any $\Psi \in H$, we have $\lim _{N \rightarrow \infty} \mathrm{I}_{N}(\Psi)=0$. Since $\Psi$ is in the ITPS containing $\otimes \Phi_{k}$, given $\varepsilon>0$, lemma 3.1 implies that there exists a $K$ and $\Psi_{K} \in \bigotimes_{k=1}^{K} \mathrm{H}\left(J_{k}\right)$ such that

$$
\left\|\Psi-\Psi_{k} \otimes\left\{\bigotimes_{k=K+1}^{\infty} \Phi_{k}\right\}\right\|<\varepsilon
$$

By lemma 7.1 we have

$$
\left|\mathrm{I}_{N}(\Psi)-\mathrm{I}_{N}\left(\Psi_{K} \otimes\left\{\bigotimes_{k=K+1}^{\infty} \Phi_{k}\right\}\right)\right|<(1+\varepsilon)^{4}-1
$$

Now consider the group integral over the $N$ degrees of freedom up to and including $J_{\jmath_{m}}^{\prime}$ where $m>K$. We have 


$$
\mathrm{I}_{N}\left(\Psi_{K} \otimes\left\{\bigotimes_{k=K+1}^{\infty} \Phi_{k}\right\}\right)=\prod_{k=m}^{j_{m}} I_{k}^{\prime}\left(\Phi_{k}\right)<2^{-m}
$$

Eqs. (7.54) and (7. 55) imply htat

$$
\lim _{N \rightarrow \infty} \mathrm{I}_{N}(\Psi)=0
$$

It now follows from Eq. (7.24) and (7.25) that for any $\Phi_{1}, \Phi_{2}, \Psi_{1}, \Psi_{2} \in H$ we have

$$
\lim _{N \rightarrow \infty} \mathrm{I}_{N}\left(\Phi_{1}, \Psi_{1} ; \Phi_{2}, \Psi_{2}\right)=0 .
$$

We summarize these results as

Theorem 7.2: Let $V$ be a countably infinite dimensional inner product space with an orthogonal basis $\left\{h_{n}\right\}_{n \in J_{\infty}}$. Let $\mathrm{U}(f, g)$ be a representation of the CCRs over $V$ such that $\vee_{n} R_{n}=\mathrm{B}(H)$ where

$$
R_{n}=\left\{\left(s h_{n}, t h_{n}\right) ;-s, t<\infty\right\}^{\prime \prime}
$$

The conditions of Theorem 7. 1 is assumed to hold. If for some nonzero $\Phi_{1}, \Psi_{1}, \Phi_{2}, \Psi_{2} \in H$ we have

$$
\lim _{N \rightarrow \infty} \mathrm{I}_{N}\left(\Phi_{1}, \Psi_{1} ; \Phi_{2}, \Psi_{2}\right)=\left(\Psi_{1}, \Psi_{2}\right)\left(\Phi_{2}, \Phi_{1}\right)
$$

independent of the ordering of the basis vectors $h_{n}$, then the representation is a tensor producl representation.

\section{Entire Function Spaces}

In this section we generalize the exponential space defined in Sec. 5 to an entire function space (Theorem 8.1). We discuss the properties of these spaces, and give an explicit construction.

Lemma 8.1: If the matrices $A_{i j}$ and $B_{i j}$ are positive-definite (positivesemidefinite), then the matrix $C_{i j}=A_{i j} B_{i j}$ is positive-definite (positive-semidefinite).

Proof: The matrix $C$ is the restriction of the Kronecker product $A \otimes B$ to the subspace spanned by the "diagonal" basis vectors $e_{j} \otimes e_{j}$.

Q.E.D. 
Lemma 8. 2: Let $A_{i j}$ be a positive-semidefinite matrix. Let $f(z)=$ $\sum a_{n} z^{n}, a_{n} \geqslant 0$ be an entire function. Then $f_{i j}(A)$ is a positive-semidefinite matrix. If $a_{n}>0$ for all $n$ then $f\left(A_{i j}\right)$ is positive-definite unless $A_{i j}=A_{i j}=$ $A_{j i}=A_{j j}$ for some pair $i \neq j$.

Proof: The matrix $A_{i j}^{0}=1$ is positive-semidefinite. By the preceding lemma, the matrix $A_{i j}^{n}$ is positive-semidefinite. Thus $\mathrm{f}\left(A_{i j}\right)$ is the sum of positive-semidefinite matrices which proves that $\mathrm{f}\left(A_{i j}\right)$ is positivesemidefinite.

We now assume $a_{n}>0$. Suppose $\sum_{j} \mathrm{f}\left(A_{i j}\right) x_{j}=0$ for $x \neq 0$. Let $k$ be the $j$ for which $A_{j j}$ is maximum among those $j$ with $x_{j} \neq 0$. Since $A_{i j}^{n}$ is positive-semidefinite we have $\sum_{j} A_{i j}^{n} x_{j}=0$ for $n=0,1,2, \ldots$. Hence $\sum_{j \neq k} \mathrm{P}\left(A_{i j}\right) x_{j}=0$ for all polynomials $\mathrm{P}$. Let $\mathrm{P}$ be a polynomial such that $\mathrm{P}\left(A_{k k}\right) \neq 0$ and $\mathrm{P}\left(A_{i j}\right)=0$ for all $A_{i j} \neq A_{k k}$. We then have $\mathrm{P}\left(A_{k k}\right) x_{k}+$ $\sum \mathrm{P}\left(A_{k j}\right) x_{j}=0$. Since $x_{k} \neq 0$, there must be some $j \neq k$ such that $A_{k j}=A_{k k}$. Because $A_{i j}$ is positive-semidefinite, the submatrix

$$
\left(\begin{array}{cc}
A_{k k} & A_{k_{j}} \\
A_{j k} & A_{j j}
\end{array}\right)
$$

is positive-semidefiinte. It follows that $A_{j k}=A_{k j}^{*}=A_{k k}^{*}=A_{k k} \geqslant A_{j j}$, and hence we must have $A_{j j}=A_{k k}$. Q.E.D.

Theorem 8.1: Let $K$ be a Hilbert space, and let $\mathrm{f}(z)=\sum a_{n} z^{n}, a_{n} \geqslant 0$ be an entire function. Then there exists a Hilbert space $\mathrm{f}(K)$ and a strongly continuous map from $K$ into a total set in $\mathrm{f}(K)$ such that

$$
(\mathrm{f}(\Phi), \mathrm{f}(\Psi))_{\mathrm{f}(K)}=\mathrm{f}\left((\Phi, \Psi)_{K}\right)
$$

If $a_{n}>0$ for all $n$, then any finite set of $\mathrm{f}\left(\Phi_{i}\right)$ is linearly independent if $\Phi_{i} \neq \Phi_{j}$ for $i \neq i$. If $K$ is separable then $\mathrm{f}(K)$ is separable.

Proof: We construct a prehilbert space $H_{0}$ as follows. Let $H_{0}$ be the linear space of all expressions of the form

$$
\sum_{i=1}^{n} c_{i} \mathrm{f}\left(\Phi_{i}\right), \quad \Phi_{i} \in K
$$

where the $c_{i}$ are complex numbers. For any $\Phi_{i}, i=1, \cdots, n$ the matrix 
$\left(\Phi_{i}, \Phi_{i}\right)$ is positive-semidefinite. By lemma 8.2 the matrix $f\left(\Phi_{i}, \Phi_{j}\right)$ is positive-semidefinite and the equation

$$
\left.\left(\sum_{i=1}^{n} c_{i} f\left(\Phi_{i}\right), \sum_{j=1}^{m} d_{j} f\left(\Psi_{j}\right)\right)=\sum_{i, j=1}^{n, m} c_{i}^{*} d_{j} \mathrm{f}\left(\Phi_{i}, \Psi_{j}\right)\right)
$$

defines an inner product in $H_{0}$. If $a_{n}>0$ for all $n$ then by lemma 8.2 the matrix $\mathrm{f}\left(\left(\Phi_{i}, \Phi_{j}\right)\right)$ is positive-definite unless $\left(\Phi_{i}, \Phi_{i}\right)=\left(\Phi_{i}, \Phi_{j}\right)=$ $\left(\Phi_{j}, \Phi_{i}\right)=\left(\Phi_{j}, \Phi_{j}\right)$, which implies that $\Phi_{i}=\Phi_{j}$. This proves that any finite set of $\mathrm{f}\left(\Phi_{i}\right)$ is linearly independent if $\Phi_{i} \neq \Phi_{j}$ for all $i \neq i$. We now define $\mathrm{f}(K)$ as the Hilbert space obtained from $H_{0}$ by the usual construction. It follows from Eq. (8.1) that the map $\Phi \rightarrow f(\Phi)$ is strongly continuous. If $K$ is separable then there is a countable set $K_{0}$ which is dense in $K$. Since $\mathrm{f}$ is strongly continuous the countable set $\left\{\mathrm{f}(\Phi) ; \Phi \in K_{0}\right\}$ is total in $\mathrm{f}(K)$, hence $\mathrm{f}(K)$ is separable. Q.E.D.

We now give a brief discussion of linear operators in $f(K)$. For any $T \in \mathrm{B}(K)$ we define a possibly unbounded linear operator on the total set of all $\mathrm{f}(\Phi)$ by

$$
\mathrm{f}(T) \mathrm{f}(\Phi)=\mathrm{f}(T \Phi)
$$

which can be extended by linearity to the prehilbert space $H_{0}$ given in the proof of Theorem 8.1. If $T$ is isometric on $K$, it follows from Eq. (8.3) that $\mathrm{f}(\mathrm{T})$ is isometric on $H_{0}$ and can therefore be extended by continuity to an isometric operator on $\mathrm{f}(K)$. If $\|T\|>1$ then $\mathrm{f}(\mathrm{T})$ is unbounded unless $\mathrm{f}(z)$ is a polynomial, since

$$
\|\mathrm{f}(T) \mathrm{f}(\lambda \Phi)\|^{2} /\|\mathrm{f}(\lambda \Phi)\|^{2}=\mathrm{f}\left(\lambda^{2}\|T \Phi\|^{2}\right) / \mathrm{f}\left(\lambda^{2}\|\Phi\|^{2}\right)
$$

which $\rightarrow \infty$ as $\lambda \rightarrow \infty$ if $\|T \Phi\|>\|\Phi\|$ unless $\mathrm{f}(z)$ is a polynomial. If $\|T\|<1$ then $f(T)$ is bounded, as can be seen most easily by the following explicit construction of $\mathrm{f}(K)$.

Given $\mathrm{f}(z)=\sum a_{n} z^{n}>0, a_{n} \geqslant 0$ and $K$ let

$$
H=\bigoplus_{\substack{n=0 \\ a_{n} \neq 0}}^{\infty}(\otimes K)_{\mathrm{s}}^{n}
$$

and consider the vectors 


$$
\tilde{\mathrm{f}}(\phi)=\oplus\left(a_{n}\right)^{1 / 2}(\otimes \phi)^{n}
$$

The proof of lemma 5.1 can easily be modified to show that the $\tilde{\mathrm{f}}(\phi)$ are a total set in $H$ if $a_{n}>0$. We have

$$
(\tilde{\mathrm{f}}(\phi), \tilde{\mathrm{f}}(\psi))=\sum a_{n}(\phi, \psi)=\mathrm{f}((\phi, \psi))
$$

Thus the mapping from $\mathrm{f}(K)$ to $H$ given by

$$
\tilde{\mathrm{f}}(\phi) \rightarrow \tilde{\mathrm{f}}(\phi)
$$

is isometric. The operator $\mathrm{f}(T), T \in \mathrm{B}(K)$ defined by Eq. (8.4) is now given by (possibly a restriction of)

$$
\mathrm{f}(T)=\oplus(\otimes T)^{n}
$$

It follows that $\mathrm{f}(T)$ is bounded if $\|T\| \leqslant 1$.

\section{Discussion of Results}

We have obtained a partial classification (up to unitary equivalence) of complete Boolean algebras $\Re$ of type I factors. Any such $\mathfrak{R}$ can be decomposed into a complete atomic Boolean algebra $\Re_{a}$ with atoms $\left\{R_{a}\right\}_{a \in A}$ and a complete nonatomic Boolean algebra $\Re_{\mathrm{c}}$. Theorem 4.1 states that $\left\{R_{a j}\right\}_{\alpha \in A}$ is a tensor product factorization. That is we can write $H=\otimes H_{\alpha}$, $R_{a}=\mathrm{B}\left(H_{\omega}\right) \otimes\left(\underset{\alpha^{\prime} \neq \alpha}{\otimes} \mathbb{1}_{\alpha^{\prime}}\right)$ and $\Re_{\mathrm{a}}$ is determined up to unitary equivalence by $\operatorname{dim} H_{a}$. Theorem 6.1 establishes a one-to-one correspondence between the unitary equivalence classes of complete nonatomic Boolean algebras of tyepe I factors satisfying the condition $\inf _{B} \mathrm{~d}(\Psi ; B)>0$ for all $\Psi \in H$, and complete nonatomic Boolean algebras of projections. For the general case the problem of classifying nonatomic Boolean algebras of projections has not been solved. But on separable Hilbert spaces one can obtain a direct integral decomposition and thus an explicit set of unitary invariants. We have also determined all factorizable vectors and all factorizable bounded linear operators for any complete Boolean algebra of type I factors satisfying the above condition. An application to the group integral for the representations of the CCRs has been given. 
These results are only a first step towards the problem of classifying all Boolean algebras of factors (or equivalently all fatorizations). Since a complete classification of non-type I factors does not yet exist, the most that one could hope for at the moment would be a classification of Boolean albgebras of type I factors. In particular, we hope to be able to eliminate the assumption $\inf _{B} \mathrm{~d}(\Psi ; B)>0$, and thus obtain a complete classification of all complete Boolean algebras of type I factors.

In a future paper a further application to the determination of all representations of the CCRs with a translationally invariant state such that all $\mathrm{R}(B)$ are type I where

$$
\mathrm{R}(B)=\{\mathrm{U}(f, g): f \text { and } g \text { have support in } B\}^{\prime \prime}
$$

and $B$ is any open subset of $n$-dimensional Euclidean space, will be given.

\section{ACKNOWLEDGEMENT}

A part of this work has been made during the first named author's visit to the University of Maryland. The first named author would like to thank Professor John S. Toll and members of Department of Physics and Astronomy for their warm hospitality.

\section{Appendix 1: Computation of the Norm of $T(\psi, Q, \chi)$}

Given a Hilbert space $K$, the equation

$$
\mathrm{T}(\psi, Q, \chi) \mathrm{e}^{\phi}=e^{(x, \phi)} \mathrm{e}^{\mathrm{Q} \phi+\psi}
$$

where $\psi, \chi \in K, Q \in \mathrm{B}(K)$ defines a possibly unbounded linear operator on the dense subspace $H_{0}$ of $\mathrm{e}^{K}$ of finite linear combinations of product vectors (see Eqs. (5.39-52)). On $H_{0}$ we have

$$
\mathrm{T}(\psi, Q, \chi)^{*}=\mathrm{T}\left(\chi, Q^{*}, \psi\right)
$$

and

$$
\mathrm{T}\left(\psi_{1}, Q_{1}, \chi_{1}\right) \mathrm{T}\left(\psi_{2}, Q_{2}, \chi_{2}\right)=e^{\left(\chi_{1}, \psi_{2}\right)} \mathrm{T}\left(\psi_{1}+Q_{1} \psi_{2}, Q_{1} Q_{2}, Q_{2}^{*} \chi_{1}+\chi_{2}\right)
$$

which can be easily verified by direct calculation. Thus we have 


$$
\begin{aligned}
\|\mid \mathrm{T}(\psi, Q, \chi)\|^{2} & =\left\|\mathrm{T}(\psi, Q, \chi)^{*} \mathrm{~T}(\psi, Q, \chi)\right\| \\
& =e^{\|\psi\|^{2}}\left\|\mathrm{~T}\left(\chi+Q^{*} \psi, Q^{*} Q, \chi+Q^{*} \psi\right)\right\|
\end{aligned}
$$

Thus it suffices to compute the norm of $\mathrm{T}(\psi, Q, \psi)$ where $Q \geqslant 0$.

Lemma A 1: If $\|Q\|>1$, then $T(\psi, Q, \psi)$ is unbounded. If $0 \leqslant Q \leqslant 1$ then

$$
\|\mathrm{T}(\psi, Q, \psi)\|=e^{\left\|(1-Q)^{-1 / 2} \psi\right\|^{2}}
$$

if $\psi$ is in the domain of $(1-Q)^{-1 / 2}$, otherwise $T(\psi, Q, \psi)$ is unnbounded.

Proof: Let $E$ be one of the spectral projections of $Q \geqslant 0$. Then $K=K_{1} \oplus K_{2}$ and $Q=Q_{1} \oplus Q_{2}$ where

$$
\begin{aligned}
& K_{1}=E K \\
& K_{2}=(1-E) K \\
& Q_{1}=E Q \\
& Q_{2}=(1-E) Q
\end{aligned}
$$

We have $\mathrm{e}^{K}=\mathrm{e}^{K_{1}} \otimes \mathrm{e}^{K_{2}}$, and $\psi=\psi_{1} \oplus \psi_{2}$ for any $\psi \in K$. It follows by direct calculation from Eq. (A1) that

$$
\mathrm{T}(\psi, Q, \psi)=\mathrm{T}\left(\psi_{1}, Q_{1}, \psi_{1}\right) \otimes \mathrm{T}\left(\psi_{2}, Q_{2}, \psi_{2}\right)
$$

Thus it suffices to compute $\|\mathrm{T}(\psi, Q, \psi)\|$ for the cases (i) $Q>1$, (ii) $Q=1$, (iii) $0 \leqslant Q<1$ with a discrete spectrum (iv) $0 \leqslant Q \leqslant 1$ with a continuous spectrum.

Case (i). We show that $\mathrm{T}(\psi, Q, \psi)$ is unbounded if $Q>1$. We have

$$
\mathrm{T}(\psi, Q, \psi) \mathrm{e}^{\lambda \phi}=e^{\lambda(\psi, \phi)} \mathrm{e}^{\lambda Q \phi+\psi}
$$

and

$$
\left\|\mathrm{e}^{\lambda \phi}\right\|^{2}=e^{|| \lambda \phi||^{2}}
$$

Then

$$
\left(\mathrm{e}^{\lambda \phi}, \mathrm{T}(\psi, Q, \psi) \mathrm{e}^{\lambda \phi}\right) /\left\|\mathrm{e}^{\lambda \phi}\right\|^{2}=\exp \left\{2 \lambda \operatorname{Re}(\psi, \phi)+|\lambda|^{2}(\phi,[Q-1] \phi)\right\}
$$

which $\rightarrow \infty$ as $|\lambda| \rightarrow \infty$.

Case (ii), $Q=1$. If $\psi \neq 0$, then there is a $\phi \in K$ such that $(\psi, \phi)>0$ and Eq. (A12) is still unbounded as $\lambda \rightarrow \infty$. But $T(0,1,0)=1$. 
Case (iii), $0 \leqslant Q<1$ with discrete spectrum. Let $h_{i}$ be a basis for $K$ such that

$$
Q h_{i}=e^{\lambda}{ }_{i} h_{i}, \quad \lambda_{i}<0
$$

(it is more convenient to write the eigenvaluses as $e^{\lambda}{ }_{i}$, see Eq. (A22) below. $\lambda_{i}=-\infty$ allowed.) Let $K_{i}$ be the one-dimensional space spanned by $h_{i}$. Then by Theorem 5.1

$$
\mathrm{e}^{K}=\otimes \mathrm{e}^{K_{i}}
$$

is the ITPS containing the vector

Let

$$
\Omega=\otimes \Omega_{i}
$$

$$
\psi=\sum k_{i} h_{i}
$$

Then we can write

$$
\mathrm{T}(\psi, Q, \psi)=\otimes \mathrm{T}_{i}\left(k_{i}, e^{\lambda}, k_{i}\right)
$$

We have

$$
\mathrm{e}^{K_{i}}=\bigoplus_{n=0}^{\infty}\left(\otimes K_{i}\right)_{\mathrm{s}}^{n}
$$

where $\left(\otimes K_{i}\right)_{\mathrm{s}}^{n}$ is the one-dimensional space spanned by $\left(\otimes h_{i}\right)^{n}$. We define an operator $a_{i}^{*}$ by

$$
a_{1}^{*}\left(\otimes h_{i}\right)^{n}=(n+1)^{1 / 2}\left(\otimes h_{i}\right)^{n+1}
$$

The operators $a_{i}, a_{i}^{*}$ are the annihilation and creation operators for the irreducible Schrödinger representation of the commutaion relations for one degree of freedom. The direct sum decompostion given by Eq. (A19) diagonalizes the number operator $N_{i}=a_{i}^{*} a_{i}$ as

$$
N_{i}=\bigoplus_{n=0}^{\infty} n
$$

By direct calculation on the total set of vectors $\mathrm{e}^{\alpha a_{i}^{*}} \Omega_{i}$ in $\mathrm{e}^{K_{i}}$ one can verify that

$$
\mathrm{T}_{i}\left(k_{i}, e^{\lambda_{i}}, k_{i}\right)=\mathrm{e}^{k_{i} a_{i}^{*}} \mathrm{e}^{\lambda_{i} a_{i}^{*} a_{i}} \mathrm{e}^{k_{i}^{*} a_{i}}
$$

Thus our problem is reduced to comguting the norm of the operator $\mathrm{e}^{k a^{*}} \mathrm{e}^{\lambda a^{*} a} \mathrm{e}^{k^{*} a}$ in the Schrödinger representation of the commutation relations for one degree of freedom. In appenidx 2 we prove that 


$$
\mathrm{e}^{k a^{*}} \mathrm{e}^{\lambda a^{*} a} \mathrm{e}^{k^{*} a}=e^{|k|^{2} /\left(1-e^{\lambda}\right)} \mathrm{e}^{\lambda b^{*} b}, \quad \lambda<0
$$

where

$$
b=a-k /\left(1-e^{\lambda}\right)
$$

We have

$$
\left[b, b^{*}\right]=+1
$$

and $b, b^{*}$ are also annihilation and creation operators for the Schrödinger representation. Since $b^{*} b$ is a number operator with eigenvalues $0,1,2, \cdots$ and $\lambda<0$ it follows that

$$
\left\|\mathrm{e}^{\lambda b^{* b}}\right\|=1, \quad \lambda<0
$$

It now follows from Eqs. (A22) and (A23) that

$$
\left\|\mathrm{T}_{i}\left(k_{i}, e^{\lambda_{i}}, k_{i}\right)\right\|=e^{\left|k_{i}\right|^{2 /\left(1-e^{\lambda_{i}}\right)}}, \quad \lambda_{i}<0
$$

Eq. (A5) now follows from Eqs. (A 14), (A17), (A 18), and (A27).

Case (iv), $0 \leqslant Q \leqslant 1$ with continuous spectrum. Consider the spectral decompostion

$$
Q=\int_{-\infty}^{0} e^{\lambda} \mathrm{d} E(\lambda)
$$

Let

$$
\begin{aligned}
& E_{n m}=\int_{-m / n}^{-(m-1) / n} \mathrm{~d} E(\lambda) \\
& Q_{n}=\sum_{m=1}^{\infty} e^{-m / n} E_{n m}
\end{aligned}
$$

Since $Q_{n} \rightarrow Q$ in norm, we have

$$
\lim \mathrm{T}\left(\psi, Q_{n}, \psi\right) \mathrm{e}^{\phi}=\mathrm{T}(\psi, Q, \psi) \mathrm{e}^{\phi}
$$

for each $\phi$. Furthermore, (A 5) for the case (iii) yields

$$
\lim \left\|\mathrm{T}\left(\psi, Q_{n}, \psi\right)\right\|=\exp \left\|(1-Q)^{-1 / 2} \psi\right\|^{2}
$$

Thus $\left\|\mathrm{T}\left(\psi, Q_{n}, \psi\right)\right\|$ is bounded and the convergence in (A31) for a total set $\mathrm{e}^{\phi}$ implies

$$
\lim \mathrm{T}\left(\psi, Q_{n}, \psi\right)=\mathrm{T}(\psi, Q, \psi)
$$

For any unit vector $\chi$, we have 


$$
\begin{gathered}
|(\chi, \mathrm{T}(\psi, Q, \psi) \chi)|=\lim \left|\left(\chi, \mathrm{T}\left(\psi, Q_{n}, \psi\right) \chi\right)\right| \\
\leqq \\
\leqq
\end{gathered}
$$

Hence $\mathrm{T}(\psi, Q, \psi)$ is bounded and

$$
\begin{aligned}
\|\mathrm{T}(\psi, Q, \psi)\| & \leqslant \lim _{n \rightarrow \infty}\left\|\mathrm{T}\left(\psi, Q_{n}, \psi\right)\right\| \\
& =e^{\left\|(1-Q)^{-1 / 2} \psi\right\|^{2}}
\end{aligned}
$$

To prove the converse inequality, consider the spectral resolution

$$
Q=\int_{0}^{1} k \mathrm{~d} E^{\prime}(k)
$$

and let

$$
\psi_{\lambda}=\int_{0}^{\lambda}(1-k)^{-1} \mathrm{~d} E^{\prime}(k \psi)
$$

Let $\chi=\mathrm{e}^{\psi} \lambda$, then

$$
\begin{aligned}
& (\chi, \mathrm{T}(\psi, Q, \psi) \chi) /\|\chi\|^{2} \\
& \quad=\exp \left\{\left(\psi, \psi_{\lambda}\right)+\left(\psi_{\lambda}, \psi\right)+\left(\psi_{\lambda}, Q \psi_{\lambda}\right)-\left(\psi_{\lambda}, \psi_{\lambda}\right)\right\} \\
& \quad=\exp \left\{\int_{0}^{\lambda}\left[2(1-k)^{-1}+k(1-k)^{-2}-(1-k)^{-2}\right] \mathrm{d}\left(\psi, E^{\prime}(k) \psi\right)\right\} \\
& \quad=\exp \left\{\int_{0}^{\lambda}(1-k)^{-1} \mathrm{~d}\left(\psi, E^{\prime}(k) \psi\right)\right\}
\end{aligned}
$$

It follows that

$$
\|\mathrm{T}(\psi, Q, \psi)\| \geqslant e^{\left\|(1-Q)^{-1 / 2} \psi\right\|^{2}}
$$

Eqs. (A 35) and (A 39) give the desired result. Q.E.D.

\section{Appendix 2: Derivation of Eq. (A23)}

It is sufficient to prove that Eq. (A23) holds on a total set. We begin with a heuristic derivation. Consider the following Ansatz.

$$
\mathrm{e}^{k(t) a^{*}} e^{\lambda(t) a^{*} a} \mathrm{e}^{k(t)^{*} a}=\mathrm{e}^{t\left(a^{*}+a *\right)(a+a)} \mathrm{e}^{c(t)}
$$

where $t, k(t), \lambda(t), c(t), \alpha$ are complex numbers. We differentiate both sides and use the identities

$$
\begin{aligned}
& \mathrm{e}^{-B a^{*} a} a^{*} \mathrm{e}^{B a^{*} a}=e^{-B} a^{*} \\
& \mathrm{e}^{-B a} a^{*} \mathrm{e}^{B a}=a^{*}-B
\end{aligned}
$$


where $B$ is any complex number. Then we obtain

$$
\begin{aligned}
\mathrm{e}^{k(t) a^{*}} \mathrm{e}^{\lambda(t) a^{*} a} \mathrm{e}^{k(t) * a}\left[k^{\prime}(t) e^{-\lambda(t)}\left(a^{*}-k(t)^{*}\right)\right. \\
\left.+\lambda^{\prime}(t)\left(a^{*}-k(t)^{*}\right) a+k^{\prime}(t)^{*} a\right] \\
=e^{c(t)} e^{t\left(a^{*+}+a^{*}\right)(a+a)}\left[\left(a^{*}+\alpha^{*}\right)\left(a+\alpha^{*}\right)+c^{\prime}(t)\right]
\end{aligned}
$$

Using Eq. (A40) to eliminate the expoonential operators, and comparing the coefficients of the operators $1, a, a^{*}, a^{*} a$ on the left and right hand sides of Eq. (A43) we find

$$
\begin{aligned}
-k^{\prime}(t) k(t)^{*} e^{-\lambda(t)} & =|\alpha|^{2}+c^{\prime}(t) \\
k^{\prime}(t)^{*}-\lambda^{\prime}(t) k(t)^{*} & =\alpha^{*} \\
k^{\prime}(t) e^{-\lambda(t)} & =\alpha \\
\lambda^{\prime}(t) & =1
\end{aligned}
$$

Using the initial conditions $k(0)=\lambda(0)=c(0)=0$, we can integrate these equations to find

$$
\begin{aligned}
& \lambda(t)=t \\
& k(t)=\alpha\left(e^{t}-1\right) \\
& c(t)=-|\alpha|^{2}\left(e^{t}-1\right)
\end{aligned}
$$

If we now set $t=\lambda$ and $\alpha=k /\left(e^{\lambda}-1\right)$ we obtain Eq. (A23). To actually prove Eq. (A23) one can verify the above derivation in the opposite direction, each equation being valid on the one-dimensional subspaces with finite particle number $a^{*} a=0,1,2, \cdots$.

Note added in proof: Lemmas 4. 4, 4. 6 and a slightly strengthened version of 4.7 can be proved in one step, with the aid of lemmas 4.1,6.2, 3. 3 and 4. 3 . 